
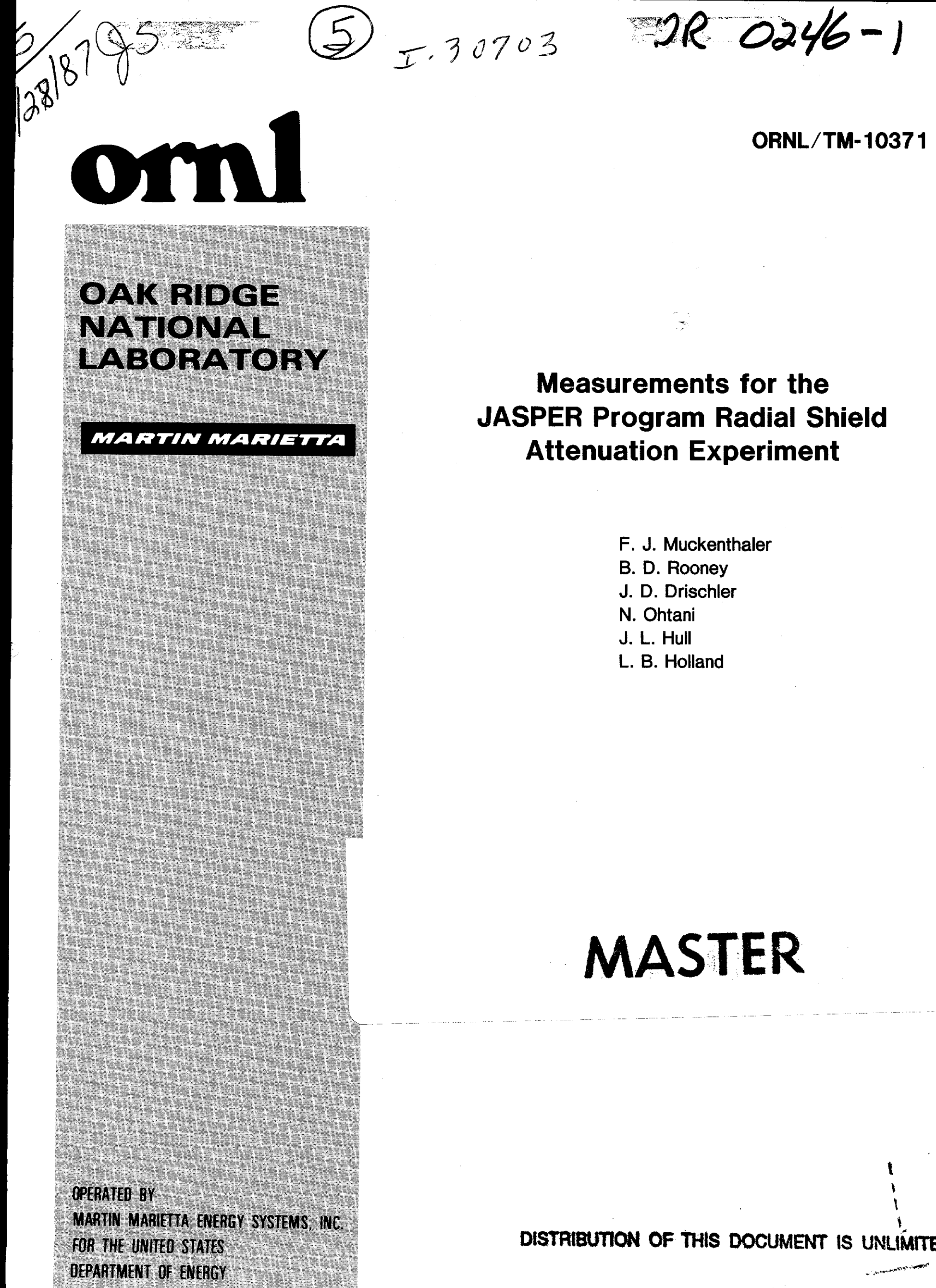

\title{
Measurements for the JASPER Program Radial Shield Attenuation Experiment
}
F. J. Muckenthaler
B. D. Rooney
J. D. Drischler
N. Ohtani
J. L. Hull
L. B. Holland

\section{MASTER}


Printed in the United States of America. Available from

the U.S. Department of Energy

Technical Information Center

P.O. Box 62, Oak Ridge, Tennessee 37830

This report was prepared as an account of work sponsored by an agency of the United States Government. Neither the U nited States Government nor any agency thereot. nor any of their employees, makes any warranty, express or implied, or assumes any legal liability or responsibility for the accuracy. completeness, or usefulness of any information, apparatus, product, or process disciosed. or represents that its use would not infringe privately owned rights. Reference herein to any specific commercial product, process, or service by trade name, trademark. manufacturer, or otherwise, does not necessarily constitute or imply its endorsement, recommendation, or favoring by the United States Government or any agency thereof. The views and opinions of authors expressed herein do not necessarily state or reflect those of the United States Government or any agency thereot. 


\section{Engineering Physics and Mathematics Division \\ MEASUREMENTS FOR THE JASPER PROGRAM RADIAL SHIELD ATTENUATION EXPERIMENT}

Report Written by: F. J. Muckenthaler

Work Done by:
F. J. Muckenthaler
B. D. Rooney
J. D. Drischler
N. Ohtani*
J. L. Hul $1^{* *}$
L. B. Holland**

Date Published: May 1987

Prepared for the

U.S. DOE office of

Liquid Metal Converter Reactor

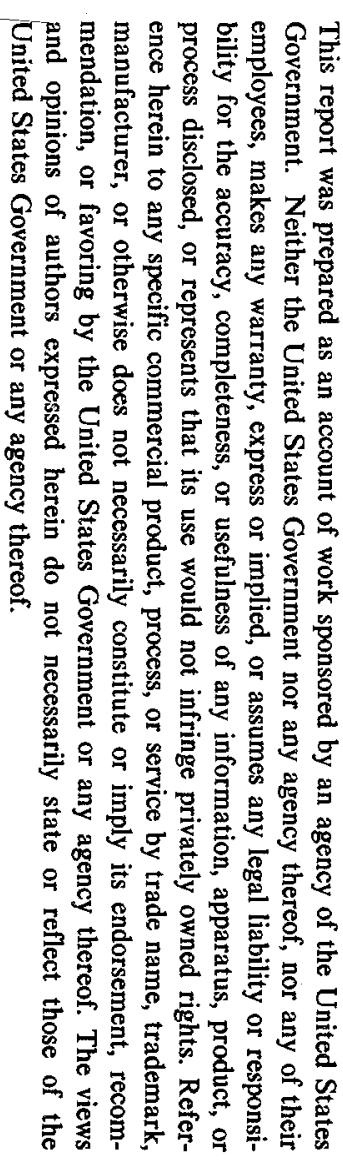

* Japan Power Reactor and Nuclear Fuel Development Corporation **operations Division

\section{DISCLAIMER}

This report was prepared as an account of work sponsored by an agency of the United States Government. Neither the United States Government nor any agency thereof, nor any of their employees, makes any warranty, express or implied, or assumes any legal liability or responsibility for the accuracy, completeness, or usefulness of any information, apparatus, product, or process disclosed, or represents that its use would not infringe privately owned rights. Reference herein to any specific commercial product, process, or service by trade name, trademark, manufacturer, or otherwise does not necessarily constitute or imply its endorsement, recommendation, or favoring by the United States Government or any agency thereof. The views and opinions of authors expressed herein do not necessarily state or reflect those of the United States Government or any agency thereof.

\section{Prepared by the}

Oak Ridge National Laboratory

Oak Ridge, Tennessee $\mathbf{3 7 8 3 1}$ operated by

Martin Marietta Energy Systems, Inc.

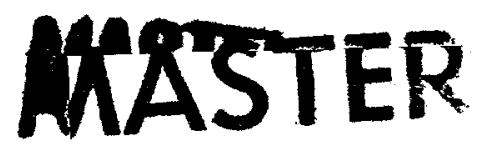
for the

U.S. DEPARTMENT OF ENERGY

under Contract No. DE-AC05-840R21400 


\section{DISCLAMMER}

Portions of this document may be illegible in electronic image products. Images are produced from the best available original document. 
TABLE OF CONTENTS

Abstract . . . . . . . . . . . . . . . . . . . . . . 1

1. Introduction . . . . . . . . . . . . . . . . . . . . . 1

2. Instrumentation. . . . . . . . . . . . . . . . . . . . . . 3

3. Experimental Configuration . . . . . . . . . . . . . . . . . 4

3.1 Spectrum Modifier (Phases I, II, III, IV, VI, VII). . . . . 4

3.2 Spectrum Modifier (Phase V) . . . . . . . . . . . . . . . 5

3.3 Stainless Steel Slabs . . . . . . . . . . . . . . . . . . . 6

3.4 Boron Carbide Slabs . . . . . . . . . . . . . . . . . . . . 6

3.5 Sodium Slabs. . . . . . . . . . . . . . . . . . . . . . . 6

3.6 Aluminum Slabs. . . . . . . . . . . . . . . . . . . . . . . 6

3.7 Graphite Slabs. . . . . . . . . . . . . . . . . . 7

4. Measurements . . . . . . . . . . . . . . . . . . . . . . 8

4.1 Spectrum Modifier (Item IA) . . . . . . . . . . . . . . . 8

4.2 Near-Core Shield Configuration - SS $+\mathrm{B}_{4} \mathrm{C}$ (Items IIA-IIE) . 8

4.3 Benchmark Experiment - SS $+\mathrm{B}_{4} \mathrm{C}$ (Phase IIIA-IIIE) . . . . . 9

4.4 Tank Type Radial Shield - Graphite $+B_{4} C$ (Items IVA-IVM). . 10

4.5 IHX Shield - SS $+\mathrm{B}_{4} \mathrm{C}$ (Items VA-VJ). . . . . . . . . . . 13

4.6 Graphite Benchmark - SS + Graphite (Items VIA-VIF). . . . . 16

4.7 Near Core Shield - SS $+\mathrm{B}_{4} \mathrm{C}$ (Items VIIA-VIID) . . . . . 17

5. Analysis of Experimental Errors. . . . . . . . . . . . . . . . . . 19

References . . . . . . . . . . . . . . . . . . 21

Acknowledgements . . . . . . . . . . . . . . . . . . . . . . . . . 21

Appendix A. Experimental Program Plan for the JASPER Radial Shield Attenuation Experiment . . . . . . . . . . . . . . . . . . . . . . . 23

Appendix B. Tables of Data. . . . . . . . . . . . . . . . . . . . . 39

Appendix C. List of Figures . . . . . . . . . . . . . . . . 71 
ABSTRACT

The Radial Shield Attenuation Experiment was conducted at the Oak Ridge National Laboratory Tower Shielding Facility during FY 1986 to: (1) provide data for calculating the shielding effectiveness of combinations of stainless steel, graphite, and boron carbide shield designs; (2) verify the accuracy of related radiation transport methods and nuclear data; and (3) substantiate the effectiveness of shield designs currently proposed by advanced LMR designers in Japan and the United States. The Tower shielding Reactor source was modified to represent neutron spectra at a specified location near the core and in the sodium pool of a typical liquid-metal-cooled reactor. The experimental configurations resulted from successive additions of the various layers of material as specified in the program plan. Integral neutron fluxes were measured behind each of the configurations at specified locations, and neutron spectra were obtained for selected mockups. The experimental data are presented in both tabular and graphical form.

This experiment is the first in a series of six experiments to be performed as part of a cooperative effort between the United States Department of Energy and the Japan Power Reactor and Nuclear Fuel Development Corporation. The research program is intended to provide support for the development of advanced sodium-cooled reactors.

\section{INTRODUCTION}

In September 1985, an agreement was signed between the United States Department of Energy and the Japanese Power Reactor and Nuclear Fuel Development Corporation (PNC) to perform a series of experiments at the oak Ridge National Laboratory's Tower shielding Facility (TSF). The research program, entitled "JASPER" for Japanese-American Shielding Program of Experimental Research, is to be a cooperative effort designed to meet the needs of both participants. All of the experiments performed will be planned jointly by ORNL and PNC representatives and derived through an iterative process of submitted proposals and mutual agreement.

The first of the six planned experiments, entitled the Radial shield 
Attenuation Experiment (started March 1986) was designed to investigate neutron transmission through benchmark and representative mockups of radial shield designs for advanced sodium-cooled reactor concepts. A lengthy program was planned consisting of nine separate investigations, some of which were given a relatively low priority. During the period of eight months alloted to do the experiment, only the top seven priority items were completed.

In this experiment, two spectrum modifiers were used to alter the energy distribution of the TSF beam, one to provide a typical near-core neutron spectrum, the other to give a spectrum for studying IHX shields in the sodium pool of a typical advanced LMR. For the near-core source spectrum, a modifier of iron, aluminum, boral and a "radial blanket" of natural $\mathrm{UO}_{2}$ was placed in the beam. For a source spectrum near the IHX, the "radial blanket" was replaced with $183 \mathrm{~cm}$ of sodium.

The components used in the experimental mockups were slabs of stainless steel, graphite, boron carbide, boral, and sodium, with aluminum slabs placed at selected locations within the mockups to represent thin sodium regions in the radial shield designs, All slabs were $1.5 \mathrm{~m}$ on a side, with varying thicknesses. The experimental program plan (Appendix A) describes the configurations and the measurements that were made.

The experimental shields were positioned in the horizontal beam emerging from TSR-II, preceded by the spectrum modifiers. Shield effectiveness was investigated through spectral and integral neutron flux measurements behind successive layers of the mockup as they were added to the configurations. Measurements were also made behind selected configurations with several Japanese ${ }^{3}$ He Bonner ball detectors at the same locations as ORNL Bonner ball measurements, but the Japanese data will not be included in this report. However, these measurements are included in the program plan (Appendix A). 


\section{INSTRUNGRTATION}

The TSF Bonner ball detection system consists of a series of detectors (polyethylene balls), each of which measures an integral of the neutron flux weighted by the energy-dependent response function for that ball. The detection device of a Bonner ball consists of a 5.1-cm-diameter spherical proportional counter filled with $\mathrm{BF}_{3}$ gas $\left({ }^{10} \mathrm{~B} / \mathrm{B}\right.$ concentration $\left.=0.96\right)$ to a pressure of 0.5 atmospheres. In order to cover a range of neutron energies, the counter is used bare, covered with cadmium, or enclosed in various thicknesses of polyethylene shells surrounded by cadmium, each detector being identified by the diameter of its shell. Bonner ball experimental results are predicted analytically by folding a calculated neutron spectrum with the Bonner ball response functions determined by Maerker et al. ${ }^{1}$ and C. E. Burgart et al. ${ }^{2}$

The NE-213 liquid scintillator spectrometer covered the neutron spectral region from about $800 \mathrm{keV}$ to $15 \mathrm{MeV}$. The pulse-height data obtained with the spectrometer were unfolded with the FERD $\operatorname{code}^{3}$ to yield absolute neutron energy spectra.

The spherical proton-recoil counters, filled with hydrogen to pressures of 1,3 , and 10 atmospheres, covered the neutron energy range from about $50 \mathrm{keV}$ to $1 \mathrm{MeV}$. Pulse-height data from the counters were unfolded with the SPEC-4 code, ${ }^{4}$ which uses the unfolded NE-213 neutron spectrum for the high energy input.

The measurements for each detector were referenced to the reactor power (watts) using the data from two fission chambers positioned along the reactor centerline as a basis. The response of these chambers as a function of reactor power level was established previously through several calorimetric measurements of the heat generated in the reactor during a temperature equilibrium condition (heat power run). 


\section{EXPERIMENTAL CONFIGURATION}

The experimental program plan (see Appendix A) specified neutron measurements to be made in and behind a series of mockups containing stainless steel, graphite, boron carbide, aluminum, and sodium as they were added to the configuration. These mockups were representative of current radial shield designs near the core and in the area of the heat exchanger. The neutron source was the TSR-II, modified to provide an incident spectrum at each location similar to that predicted by calculations for the LMR design. Thus, two different modifiers were used. Preanalysis calculations ${ }^{5}$ for the experiment fixed the composition and thickness of the modifiers and contributed to the design of the shield configurations themselves, all of which are described below. It should be noted that the material thicknesses given in the program plan (Appendix A) are nominal; the actual thicknesses on centerline are stated in the schematics.

\subsection{SPECTRUM MODIPIER (Phases I, II, III, IV, VI, VII)}

The preanalysis calculation indicated that nominally $10 \mathrm{~cm}$ of iron, followed by $10 \mathrm{~cm}$ of aluminum, $2.5 \mathrm{~cm}$ of boral, and $20.3 \mathrm{~cm}$ of "radial blanket" placed in the TSR-II beam would provide a spectrum of neutrons representative of those incident on the radial shield surrounding the LMR core. In the experiment (see Figure 1) the iron component consisted of two slabs 5.16 - and $5.11-\mathrm{cm}-\mathrm{thick}$, both $152.4 \mathrm{~cm}(60-\mathrm{in})$ on an edge. The three aluminum slabs totalled $9.17 \mathrm{~cm}$ in thickness, and the boral was $2.54-\mathrm{cm}-$ thick, all having the same edge length as the iron slabs. Composition of the iron, aluminum, and boral are given in Tables 1, 2, and 3 respectively. (Note: all tables are included in Appendix B.)

The $\mathrm{UO}_{2}$ slabs used to represent the "radial blanket" had been fabricated for earlier experiments performed in the LMFBR program. They contain natural $\mathrm{UO}_{2}$ pellets, $1.397-\mathrm{cm}$ OD, enclosed in aluminum cylinders having an oD of $1.524 \mathrm{~cm}$. Between the aluminum and the pellets there is a 0.00508 to $0.01016-\mathrm{cm}$ void filled with argon. The cylinders are stacked side-by-side vertically having a triangular pitch of $1.608 \mathrm{~cm}$. The void between the cylinders are filled with sodium. These rods are enclosed in 
iron vessels having an overall thickness of $11.05 \mathrm{~cm}$ and a length of 152.4 cm on each side.

Each of the two slabs used in this experiment contain 522 rods amounting to $64.6 \%$ of the volume of the slab. There are seven rows of rods with 74 and 75 rods per alternate row. The $\mathrm{UO}_{2}$ density is $10.28 \mathrm{~g} / \mathrm{cc}$ (94\% of theoretical). The volume of the aluminum cladding is $11.2 \%$ and that for the sodium is 23.3\%, leaving a void volume between the pellet and the aluminum cylinder of $1 \%$. The pellet stack length in each of the rods is approximately $121.9 \mathrm{~cm}$. These rods were built by Numes Corporation in 1962 to conform, in general, to the then AEC/RDT design standards for the FFTF. A schematic of the slab is shown in Figure 2, with analyses of the $\mathrm{UO}_{2}$ and aluminum given in Tables 4 and 5 .

This spectrum modifier (SM1) was surrounded by $20.3 \mathrm{~cm}$ (8-in) of lithiated parafin followed by up to $152.4 \mathrm{~cm}(60-\mathrm{in})$ of concrete to minimize the neutrons scattering back into the slabs and to reduce the amount of background radiation reaching the detectors. The lithiated paraffin was shaped as small bricks $10.16 \mathrm{~cm}$ on edge and 20.3-cm-long (4in-facing $\times$ 8-in-long) and the concrete consisted of large blocks $61 \mathrm{~cm}$ on edge and 30.5-cm-thick (24-in-facing $\times$ 12-in-thick). The composition of the lithiated paraffin and concrete blocks are presented in Tables 6 and 7 respectively.

\subsection{SPECTRUM MODIFIER (PHASE V)}

For Phase $V$ of the experiment, the radial blankets in SM1 were replaced with six slabs of nominally $30.48-\mathrm{cm}$-thick sodium to give a second spectrum modifier, SM2. The sodium was contained in aluminum cans whose faces were $0.635-\mathrm{cm}$-thick and $152.4 \mathrm{~cm}$ on an edge. It should be mentioned that these slabs had been used in earlier experiments at the TSF and down through the years several of the slabs have developed a slight bulge in the middle due to settling of the sodium. The amount of the bulge can be noted in the difference between centerline thickness measurements given in the schematics and the actual thickness of $31.75 \mathrm{~cm}(30.48 \mathrm{~cm}$ sodium plus 1.27 $\mathrm{cm}$ of aluminum) at the edge of the slabs. SM2 was also surrounded by 20.3 $\mathrm{cm}$ of lithiated paraffin followed by concrete. Composition of the sodium 
slabs is given in Table 8 , and a schematic of SM2 is shown in Figure 3.

\subsection{STAINLESS STEEL SLABS}

The stainless steel (SS) slabs used in the experimental mockups were nominally either $2.54 \mathrm{~cm}(1-\mathrm{in})$ or $5.08 \mathrm{~cm}$ thick $(2-\mathrm{in})$ and $152.4 \mathrm{~cm}(60-$ in) on an edge. These slabs are type 304 SS, whose elemental composition can be found in Table 9 .

\subsection{BORON CARBIDE SLABS}

The five boron carbide slabs used in this experiment consisted of stainless steel cans filled with 120 grit boron carbide powder. Three different thicknesses were filled, $5.08 \mathrm{~cm}(2-$ in $), 10.16 \mathrm{~cm}(4-\mathrm{in})$, and $15.24 \mathrm{~cm}(6-i n)$, denoted as 4 and $5 W, 2$ and $3 W$, and $1 W$ respectively in Figure 4. The actual thicknesses of the slabs (SS included) were measured along the centerline as indicated in the figure. Spacer pins were used in both the nominally $5-\mathrm{cm}-$ and $15.24-\mathrm{cm}$-thick slabs to maintain constant thickness over the width of the slab. All slabs were $152.4 \mathrm{~cm}(60-\mathrm{in})$ on an edge.

The densities of the $\mathrm{B}_{4} \mathrm{C}$ slabs are $1.42 \mathrm{~g} / \mathrm{cc}$ for container $1 \mathrm{~W}, 1.39$ $\mathrm{g} / \mathrm{cc}$ for container $2 \mathrm{~W}, 1.37 \mathrm{~g} / \mathrm{cc}$ for container $3 \mathrm{~W}, 1.41 \mathrm{~g} / \mathrm{cc}$ for container $4 \mathrm{~W}$, and $1.44 \mathrm{~g} / \mathrm{cc}$ for container $5 \mathrm{~W}$. An analysis of the $\mathrm{B}_{\mathbf{4}} \mathrm{C}$ powder is given in Table 10.

\subsection{SODIUM SLABS}

The sodium slabs discussed as part of the spectrum modifier in section 3.2 are the same sodium slabs that were used later as part of the shield mockups in other configurations.

\subsection{ALUMINUM SLABS}

Thin aluminum slabs $1.27 \mathrm{~cm}(0.5-i n)$ and $2.54 \mathrm{~cm}(1-i n)$, were used as part of the configurations to mockup spaces that would be occupied by sodium coolant in a typical LMR-advanced reactor design. These slabs are of the same aluminum (6061) used in the spectrum modifiers described earlier. 


\subsection{GRAPHITE SLABS}

A combination of graphite pieces, $30.48-\mathrm{cm}-(12-\mathrm{in})$ and $121.9-\mathrm{cm}-1$ ong $(48-\mathrm{in})$ and $10.16 \mathrm{~cm}(4-\mathrm{in})$ on a side, were stacked to form a slab $152.4 \mathrm{~cm}$ on an edge and $10.16-\mathrm{cm}$-thick. The pieces were precisely machined to provide good surface-to-surface contact and eliminate voids for possible neutron steaming. An analysis of the graphite is given in Table 11. 


\section{MBASUREMENTS}

\subsection{SPECTRUM MODIPIER (ITEM IA)}

Measurements of the fast neutron spectra behind SM1 were made with both the NE-213 liquid scintillator and the hydrogen-filled proton recoil detectors. Each detector was located on the beam centerline $178.8 \mathrm{~cm}$ behind the radial blanket slabs as shown in Figure 1 . The resulting energy spectra are plotted in Figures 5 and 6 and listed in Tables 12 and 13 for the NE-213 spectrometer and proton-recoll detectors, respectively. Integral neutron flux measurements with the 3-, 5-, and 10-inch diameter Bonner balls were also made on the beam centerline at the same location. These results are given in Table 14.

Traverses were made along the centerline of the configuration with the 3-, 5-, 8-, and 10-inch Bonner balls. Measurements were made at three specific locations, $30 \mathrm{~cm}$ behind the $S M$, and 304.8 and $457.2 \mathrm{~cm}$ from the reactor center. Those at 304.8 and $457.2 \mathrm{~cm}$ were made with and without a shadow shield of lithiated paraffin placed between the detector and SM1 (noted as background and foreground in the tables). The measurements at $30 \mathrm{~cm}$ are presented in Table 15, and those at 304.8 and $457.2 \mathrm{~cm}$ are given in Table 16. Finally, the traversing mechanism was rotated 90 degrees and a horizontal traverse through the configuration midplane was made with the 5-inch Bonner ball at $30 \mathrm{~cm}$ behind SM1. This data is in Table 17.

\subsection{NEAR-CORE SHIELD CONRIGURATION - SS + $B_{4} C$ (ITEMS IIA - IIE)}

The purpose of this particular phase of the experiment was to mockup a typical near-core shield for an advanced LMR concept. Measurements were made behind successive additions of the shield that included slabs of aluminum (to represent sodium), SS, and $B_{4} C$, and ending with an addition of a single slab of sodium in the last series of measurements. For the first measurements SM1 was followed by $1.27 \mathrm{~cm}$ of aluminum and $15.24 \mathrm{~cm}$ of SS (see Figure 7). The 3-, 5-, 8-, and 10-inch ORNL Bonner ball measurements were made on centerline at $30 \mathrm{~cm}$ behind the $\mathrm{SS}$ and at a point $304.8 \mathrm{~cm}$ from the reactor center that included both foreground and background runs. These results are given in Tables 15 and 16 , respectively. The traverser 
was rotated 90 degrees and a 5-inch Bonner ball horizontal traverse was made through the configuration midplane. These measurements are in Table 17.

A second slab of aluminum was added to the configuration followed by $15.24 \mathrm{~cm}$ of $\mathrm{B}_{4} \mathrm{C}$ and $2.54 \mathrm{~cm}$ SS (see Item IIB, Figure 7). Again, measurements were made with the 3-, 5-, 8-, and 10-inch Bonner balls at $30 \mathrm{~cm}$ behind the configuration and at $304.8 \mathrm{~cm}$ from the reactor center, as done in Item IIA. The data are tabulated in Tables 15 and 16 , respectively. The same measurements were made after the addition of another $1.27 \mathrm{~cm}$ of aluminum and $15.24 \mathrm{~cm}$ of $\mathrm{B}_{4} \mathrm{C}$ (see Item IIC, Figure 7). This time, however, two slabs of $\mathrm{B}_{4} \mathrm{C}$ were used to obtain that thickness, resulting in $2 \mathrm{~cm}$ of $\mathrm{ss}$ between the two nominal thicknesses of 10.16 and $5.08 \mathrm{~cm}$. These results may also be found in Tables 15 and 16 .

Spectral measurements were made on centerline at $47.3 \mathrm{~cm}$ behind the configuration following the addition of another slab of aluminum and two slabs of $\mathrm{B}_{4} \mathrm{C}, 10.16$ and $5.08 \mathrm{cms}$ (see Item IID, Figure 8). The resulting spectra are plotted in Figure 9 and listed in Table 18 for the NE-213 scintillator and in Figure 10 and Table 19 for the hydrogen counters. The three (3-, 5-, 10-inch) Bonner ball count rates at this same location are given in Table 14. Results from a 5-inch Bonner ball traverse perpendicular to the configuration centerline at $30 \mathrm{~cm}$ behind the $\mathrm{B}_{4} \mathrm{C}$ are listed in Table 17. Measurements with the 3-, 5-, 8-, and 10-inch Bonner balls were made on centerline at $30 \mathrm{~cm}$ behind the $\mathrm{B}_{4} \mathrm{C}$ and at $304.8 \mathrm{~cm}$ from the reactor center (foreground and background). The results are given in Tables 15 and 16, respectively.

The last slab added to the configuration in this phase was a $30.48 \mathrm{~cm}$ slab of sodium (see Item IIE, Figure 7). Measurements include a horizontal traverse at $30 \mathrm{~cm}$ behind the sodium with the 5 -inch Bonner ball and measurements on centerline at $30 \mathrm{~cm}$ behind the sodium and at $304.8 \mathrm{~cm}$ from the reactor center (foreground and background). These results are included in Tables 17,15 , and 16 , respectively.

\subsection{BENCHMARK EXPERIMENT - SS + $\mathrm{B}_{4} \mathrm{C}$ (PHASE IIIA - IIIE)}

The first configuration of this series was SM1 followed by 
$10.16 \mathrm{~cm}$ SS. A schematic of the mockup for this arrangement is shown in Figure 11. Axial traverses along the beam centerline were made with the 3-, 5-, 8-, and 10-inch Bonner balls at $30 \mathrm{~cm}$ behind the ss and at $304.8 \mathrm{~cm}$ from the reactor center. These results are included in Tables 15 and 16 . Again a horizontal traverse with the 5 -inch Bonner ball $30 \mathrm{~cm}$ behind the SS was made through the configuration midplane and the data are listed in Table 17.

The second configuration in this series included a $5.08 \mathrm{~cm} \mathrm{slab}$ of $\mathrm{B}_{4} \mathrm{C}$ behind the SS, Item IIIB, as shown in Figure 12. Again, measurements were made along centerline at $30 \mathrm{~cm}$ behind the $\mathrm{B}_{4} \mathrm{C}$ and at the $304.8 \mathrm{~cm}$ location. These results are given in Tables 15 and 16 . The data from the 5-inch Bonner ball horizontal traverse through configuration midplane at $30 \mathrm{~cm}$ behind the $\mathrm{B}_{4} \mathrm{C}$ are given in Table 17.

In the third mockup (see Item IIIC, Figure 11), the $5.08 \mathrm{~cm} \mathrm{~B} \mathrm{C}_{4} \mathrm{slab}$ was replaced with a second $10.16 \mathrm{~cm}$ of $\mathrm{sS}$ (total now $20.32 \mathrm{~cm}$ ). The traverses made behind the previous configuration were repeated here and the data included in Tables 15, 16, and 17.

For the mockup in Item IIID (see Figure 11), a $5.08 \mathrm{~cm}$ slab of $\mathrm{B}_{4} \mathrm{C}$ was placed behind the $20.32 \mathrm{~cm}$ of $\mathrm{ss}$. Only the centerline traverses were made behind this configuration with the four Bonner balls, again at $30 \mathrm{~cm}$ from the $\mathrm{B}_{4} \mathrm{C}$ and $304.8 \mathrm{~cm}$ from reactor center. The data are included in Tables 15 and 16 , respectively.

In the final mockup, the $\mathrm{B}_{4} \mathrm{C}$ thickness was increased to $10.16 \mathrm{~cm}$ by the addition of a second $5.08 \mathrm{~cm} s \mathrm{slab}$. Traverses were made both along the axis and perpendicular to it as described for Item IIIB. Data from the 5-inch Bonner ball traverse at $30 \mathrm{~cm}$ behind the $\mathrm{B}_{4} \mathrm{C}$ are given in Table 17 and the data from centerline traverses are listed in Tables 15 and 16.

\subsection{TANK TYPE RADIAL SHIELD - GRAPHITE + $\mathrm{B}_{\mathbf{4}} \mathrm{C}$ (ITEMS IVA - IVM)}

The configurations in this series modeled a typical radial shield design for large tank (pool) type reactor concepts. The same SM1 was used as was used in the previous series, followed by mockups using both graphite and $\mathrm{B}_{4} \mathrm{C}$.

The first configuration called for SM1 to be followed by aluminum 
(again representing sodium), ss, another slab of aluminum, and the first $10.16 \mathrm{~cm}$ of graphite, followed by $5.08 \mathrm{~cm}$ SS. This arrangement is shown in Figure 13. As in the previous series, measurements were made radially behind the configuration and along its centerline. The data from the horizontal traverse $30 \mathrm{~cm}$ behind the ss with the 5 -inch Bonner ball are given in Table 20, the measurements along the centerline at $30 \mathrm{~cm}$ and $304.8 \mathrm{~cm}$ are presented in Tables 15 and 16 respectively.

A second combination of aluminum, graphite, and Ss were added to the configuration for Item IVB, as shown in Figure 14. Only the previous centerline measurements were repeated at $30 \mathrm{~cm}$ behind the $\mathrm{ss}$ and at $304.8 \mathrm{~cm}$ from reactor center. These results are also included in Tables 15 and 16 , respectively.

In Item IVC, the third slab combination added to the configuration included aluminum, followed by $15.24 \mathrm{~cm}$ of $\mathrm{B}_{4} \mathrm{C}$ and $2.59 \mathrm{~cm}$ of $\mathrm{SS}$. For the spectral measurements it was necessary to add $7.62 \mathrm{~cm}$ of lead between the configuration and the spectrometer, as seen in Figure 15 , to attenuate the gamma-rays. The NE-213 scintillator and the hydrogen detectors were located on centerline at $42.8 \mathrm{~cm}$ beyond the $\mathrm{SS}$ (a point $256.7 \mathrm{~cm}$ from the reactor center), and $32.9 \mathrm{~cm}$ behind the lead. The spectra are plotted in Figures 16 and 17 and listed in Tables 21 and 22 . The 3-, 5-, and 10-inch Bonner ball measurements at this same location are included in Table 14 . The two lead slabs were then removed to complete the remaining Bonner ball measurements. Results from the 3-, 5-, 8-, and 10-inch Bonner ball traverses along centerline at $30 \mathrm{~cm}$ behind the $\mathrm{ss}$ and, for this configuration, at both 304.8 and $457.2 \mathrm{~cm}$ from the reactor center, are listed in Tables 15 and 16. Data from the horizontal traverse at $30 \mathrm{~cm}$ behind the configuration with the 5-inch Bonner ball are given in Table 20 . Another slab of aluminum and $5.08 \mathrm{~cm}$ SS were added to give the next configuration (see Figure 18, Item IVD). Centerline measurements were made at $30 \mathrm{~cm}$ behind the $\mathrm{SS}$, and, starting with this configuration, at $457.2 \mathrm{~cm}$ from the reactor center. This change was made to maintain a reasonable separation between the stationary point and the point $30 \mathrm{~cm}$ behind the configuration as its thickness increased. Data at $30 \mathrm{~cm}$ are listed in Table 15 and the new $457.2 \mathrm{~cm}$ point data are given in Table 16 . 
The next additions to the mockup were again slabs of aluminum, graphite, and $S S$ as seen in Figure 18, Item IVE. The centerline measurements at $30 \mathrm{~cm}$ and $457.2 \mathrm{~cm}$ were repeated and results listed in Tables 15 and 16 respectively.

With the addition of another aluminum, graphite, ss combination (Item F, Figure 18), the background count rate (shadow shield in place) at $457.2 \mathrm{~cm}$ became a major portion of the foreground count rate (shadow shield removed). The original data plan called for a continuation of the same type of measurements throughout the remainder of Phase IV, that is, Bonner ball measurements on centerline at $30 \mathrm{~cm}$ and $457.2 \mathrm{~cm}$ as in IV-E. However, the large background contribution indicated a new approach was needed to complete the remainder of the mockups. Thus, in IVF, the original measurements were completed as planned, and then repeated as close as feasible behind the configuration as asked in IVF, part 2 (see data plan). using the following procedure. A lithium hydride (LiH) slab, 30.48-cmthick, was placed directly behind the configuration and the detector positioned on centerline directly behind the LiH. Measurements were made with and without the LiH present (background and foreground respectively), keeping the detector at the same location. This substitution in procedures was followed until IVI, as seen in the program plan (Appendix A), when it became necessary once again to alter the program plan. The data obtained at $30 \mathrm{~cm}$ behind configuration IVF and at $457.2 \mathrm{~cm}$ from reactor center are given in Tables 15 and 16. The measurements at the location $72.4 \mathrm{~cm}$ behind the SS slab, with and without the LiH, can be found in Table 23.

Next, another combination of aluminum, graphite, and ss were added to the mockup (see Item IVG, Figure 18). The measurements, foreground and background, on centerline at $53.6 \mathrm{~cm}$ behind the $\mathrm{ss}$ are listed in Table 23 . Measurements were repeated after the addition of another $5.08 \mathrm{~cm} s$ lab of $\mathrm{sS}$ (Item IVH, Figure 18), this time with the detector at $52.1 \mathrm{~cm}$. The data are also included in Table 23 .

For configuration Item IVI, a $30.48 \mathrm{~cm}$ slab of sodium was added and measurements made at $49.5 \mathrm{~cm}$ behind the sodium with and without the LiH slab present. The results are listed in Table 23. Again the background count rate was a large fraction of the foreground rate, suggesting yet 
another change in the data taking process. The program plan had called for the continued additions of sodium slabs in Items IVJ through $M$, however, with the large background in IVI it was deemed not feasible. Having the same material in adjacent slabs in IVJ through $M$, namely sodium, was conducive to making in situ type measurements that could be readily compared to calculations. For the measurements, a nominal $30 \mathrm{~cm}$ void was created by placing a second sodium slab $30 \mathrm{~cm}$ behind the first one as designated in Item IVIA (see Program Plan). Doing this type of measurement requires placement of adequate shielding around the void and sodium tanks to inhibit the background neutrons. It should be noted that beginning with the addition of the sodium tanks to the mockups, only $10.16 \mathrm{~cm}(4-$ in) of lithiated parafin was placed around their edges instead of the usual $20.32 \mathrm{~cm}(8-i n)$ placed around the previous slabs. This change was due to insufficient quantities of lithiated paraffin. In addition, there was at least $45.72 \mathrm{~cm}(18-i n)$ of plain and borated polyethylene over the void for the in situ measurements, supplemented with concrete around the lithiated paraffin on the sides. Lithium hydride and borated poly were placed behind the sodium and a LiH slab served as a door for placement of the detectors in the void. Measurements for configuration IVIA were made on centerline in the void (see Figure 19) with the bare, Cd-covered, 3-, 5-, 8-, and 10inch Bonner balls and these results are given in Table 24.

Similar procedures were used to make in situ type measurements for the remainder of the configurations $(J-M)$ as listed in the program plan (see Figures 20-23). These results are included in Table 24.

\subsection{IHX SHIELD - SS + $B_{4} C$ (ITEMS VA - VJ)}

For this series of measurements, the SM was altered by removing the two slabs of radial blanket and replacing them with six slabs of sodium $(152.4 \mathrm{~cm})$. As with the previous modifier, this one was also surrounded by $20.32 \mathrm{~cm}(8-\mathrm{in}$.$) of lithiated paraffin followed by concrete (see Figure 3$ ). A horizontal traverse was made through the midplane with the 5-inch Bonner ball $30 \mathrm{~cm}$ behind the configuration. These data are in Table 25. Bare, Cd-covered, 3-, 5-, 8-, and 10-inch Bonner ball measurements were made on centerline at $30 \mathrm{~cm}$ behind the mockup and at $501: 3 \mathrm{~cm}$ from the reactor 
center (foreground and background) and these count rates are listed in Tables 26 and 27.

Thermal neutron flux measurements behind thick shields are sometimes very difficult because of the unusually large background source of thermal neutrons from air scattering and those generated in the material around the mockups. To eliminate this background would have been very difficult and expensive. However, for this series of measurements in Phase $v$, two additional measurements with the bare detector were added to the program plan to assist in determining the magnitude of the thermal flux leaving the back surface of the last slab in the configuration. These measurements, listed as $A 3$ and $A 4$ in VA of the program plan, were made behind each configuration in Phase $V$. The measurements for Item $A 3$ were made with the bare detector placed on centerline at $30 \mathrm{~cm}$ behind the last slab and surrounded by cadmium on all sides except for the side that was adjacent to the face of the last slab in the configuration. The 30-40 mil-thick cadmium was in the shape of a rectangular box, $152.4 \mathrm{~cm} \times 152.4 \mathrm{~cm}(60-$ in $\mathrm{x}$ $60-i n)$ and $45.72-\mathrm{cm}$-deep $(18-i n)$, the height and width being the same as that of the last slab. The open side of the box (no cadmium) allowed the detector to intercept thermal neutrons that emerged only from the surface of the last slab. The results from this measurement in VA are listed in Table 26.

With the bare detector on centerline at $501.3 \mathrm{~cm}$ from the reactor center a reverse of the last procedure was used. The detector was left exposed to only the background thermals by placing a sheet of cadmium over the face of the last slab in the configuration. This measurement is included in Table 27. This count rate can be subtracted from the count rate without cadmium over the face of the configuration to determine a measure of the thermal neutrons leaving the mockup.

The same measurements were repeated behind the next configuration where a $5.08 \mathrm{~cm}$ SS slab was added to the SM (Item VB), see Figure 24 . The measurements along centerline with the same group of Bonner balls at $30 \mathrm{~cm}$ and $501.3 \mathrm{~cm}$ are given in Tables 26 and 27 , respectively. The results for the 5-inch Bonner ball traverse perpendicular to the configuration centerline at $30 \mathrm{~cm}$ behind the ss are in Table 25 . 
A slab of sodium was added to the configuration in VC (Figure 24). Only centerline measurements were made at $30 \mathrm{~cm}$ and $501.3 \mathrm{~cm}$ and these results are also given in Tables 26 and 27 . For VD, a second slab of sodium was added, increasing the sodium thickness to $61 \mathrm{~cm}$. The horizontal traverse measurements at $30 \mathrm{~cm}$ behind the sodium are given in Table 25 , while the group of measurements on centerline at $30 \mathrm{~cm}$ behind the sodium and at the $501.3 \mathrm{~cm}$ location are listed in Tables 26 and 27 respectively.

The SS and sodium in the mockup were then removed and replaced (Figure 25) with $5.08 \mathrm{~cm} \mathrm{~B} \mathrm{~B}_{4} \mathrm{C}$ behind SM2 followed by $5.08 \mathrm{~cm}$ SS (Item VE). Again only centerline measurements were made at the same points as in previous measurements, $30 \mathrm{~cm}$ and $501.3 \mathrm{~cm}$. These measurements and similar measurements made behind the next configuration, VF, where a $30.48 \mathrm{~cm} \mathrm{slab}$ of sodium was included in the mockup, are given in Tables 26 and 27 , respectively. The sodium thickness was increased to $61 \mathrm{~cm}$ in Item VG, behind which were made a horizontal traverse with the 5-inch Bonner ball and centerline measurements similar to those in the previous configurations. These results are also included in their respective Tables 25,26 , and 27 .

Another change in shield design occurred in Item VH (see Figure 26), where the $61 \mathrm{~cm}$ of sodium were removed from behind the $S S$ in VG and the $5.08 \mathrm{~cm}$ thickness of $\mathrm{B}_{4} \mathrm{C}$ preceding the $\mathrm{SS}$ was increased to $10.16 \mathrm{~cm}$ $\left(5.08 \mathrm{~cm}\right.$ SS still behind $\left.B_{4} C\right)$. For Item VI, one of the $30.48 \mathrm{~cm}$ slabs of sodium was added behind the $\mathrm{B}_{4} \mathrm{C}$ and $\mathrm{SS}$. The same centerline measurements were repeated for these two mockups, with the data obtained on centerline at $30 \mathrm{~cm}$ listed in Table 26, and the measurements at $501.3 \mathrm{~cm}$ from the reactor center given in Table 27.

For the last mockup in this phase (VJ), a second slab of sodium was added (giving a total of $61 \mathrm{~cm}$ ). Measurements from the horizontal traverse through the configuration midplane with the 5 -inch Bonner ball at $30 \mathrm{~cm}$ behind the sodium are given in Table 25. Results from the measurements along centerline with the six Bonner balls at $30 \mathrm{~cm}$ behind the sodium and at $501.3 \mathrm{~cm}$ from the reactor center are listed in Tables 26 and 27 , respectively. 


\subsection{GRAPHITE BENCHIARK - SS + GRAPHITE (ITEMS VIA - VIF)}

For this series of measurements, the SM was the same as in IA (iron + aluminum + boral + radial blankets). The first mockup behind SM1 contained $1.27 \mathrm{~cm}$ aluminum, $15.24 \mathrm{~cm}$ of stainless steel, and $10.16 \mathrm{~cm}$ of graphite as shown in Figure 27. To make spectral measurements behind this mockup it was necessary to place $7.62 \mathrm{~cm}$ of lead between the graphite and the detector to attenuate the gamma-ray flux. Spectral measurements were made at $51.1 \mathrm{~cm}$ behind the graphite $(36.4 \mathrm{~cm}$ behind the lead), which corresponds to a distance of $219.3 \mathrm{~cm}$ from the reactor center. The spectrum of high energy neutrons is plotted in Figure 28 and listed in Table 28, that for the neutrons in the lower energy range is plotted in Figure 29 and listed in Table 29. The 4-, 5-, and 10-inch Bonner ball data at the same location are given in Table 14. The 4-inch Bonner ball was used for this single measurement since the 3 -inch ball was not avallable. Results from a 5-inch Bonner ball traverse horizontally through the configuration midplane are in Table 30. The bare, Cd-covered, 3-, 5-, 8-, and 10-inch Bonner ball results on centerline at $30 \mathrm{~cm}$ behind the mockup and at $304.8 \mathrm{~cm}$ from the reactor center are given in Tables 31 and 27 .

The thickness of the graphite slab was increased from $10.16 \mathrm{~cm}$ to $20.32 \mathrm{~cm}$ for the next mockup (Item VIB) in Figure 30 . The 5-inch ball horizontal traverse measurements at $30 \mathrm{~cm}$ are listed in Table 30 . The six Bonner ball measurements at $30 \mathrm{~cm}$ and $304.8 \mathrm{~cm}$ on centerline are contained in Tables 31 and 27. Adding a third graphite slab raised the total graphite thickness to $30.48 \mathrm{~cm}$ (see Item VIC, Figure 30). Centerline measurements at $30 \mathrm{~cm}$ beyond the graphite and at $304.8 \mathrm{~cm}$ with the six Bonner balls are also listed in Tables 31 and 27.

In Item VID, the graphite thickness was $40.64 \mathrm{~cm}$. The 5-inch Bonner ball data from the horizontal traverse $30 \mathrm{~cm}$ behind the mockup are in Table 30. Data with the six balls on centerline at the $30 \mathrm{~cm}$ and $304.8 \mathrm{~cm}$ positions are listed in Tables 31 and 27 . The $30 \mathrm{~cm}$ and $304.8 \mathrm{~cm}$ measurements on centerline were repeated following the addition of another $10.16 \mathrm{~cm}$ of graphite (total now $50.8 \mathrm{~cm}$ ), see Item VIE in Figure 30 . These results are also in Tables 31 and 27.

For the final mockup in this series the graphite thickness was 
increased to $60.96 \mathrm{~cm}$ (Item VIF). To make the spectral measurements it was necessary to follow the graphite with a $5.08 \mathrm{~cm}$ slab of $B_{4} C$ followed by $15.24 \mathrm{~cm}$ of lead (see figure 31 ). The $\mathrm{B}_{4} \mathrm{C}$ slab was needed to reduce the thermal and very low energy neutron capture in the lead. Without the $\mathrm{B}_{4} \mathrm{C}$ the lead was acting as a strong gamma-ray source. Spectral measurements were made on centerline at $63.2 \mathrm{~cm}$ beyond the graphite, which corresponded to $36.3 \mathrm{~cm}$ behind the lead and $282.2 \mathrm{~cm}$ from the reactor center. The NE-213 results are plotted in Figure 32 and listed in Table 32 , while those for the hydrogen counters are in Figure 33 and Table 33. The 3-, 5-, and 10-inch Bonner ball data at the same location with the lead and $\mathrm{B}_{4} \mathrm{C}$ still in the configuration are given in Table 14 . With the lead and $\mathrm{B}_{4} \mathrm{C}$ removed (see Figure 30 ) the 5 -inch ball was traversed horizontally at $30 \mathrm{~cm}$ behind the graphite and the data are shown in Table 30. The Bonner ball data on centerline at $30 \mathrm{~cm}$ behind the graphite and at $304.8 \mathrm{~cm}$ from reactor center are listed in Tables 31 and 27.

\subsection{NEAR CORE SHIELD - SS + $B_{4}$ C (ITEMS VIIA - VIID)}

The first mockup contained two sets of $1.27-\mathrm{cm}-\mathrm{aluminum}$ plus $15.24-\mathrm{cm}-$ SS slabs preceded by the same SM1 used in Item VI: iron, aluminum, boral, and radial blankets as shown in Figure 34 . In Item VIIB, a third set of aluminum-stainless steel slabs was added. For each of these mockups a horizontal traverse with the 5-inch Bonner ball was run at $30 \mathrm{~cm}$ behind the SS. The data are given in Table 30. Traverses along the centerline were run with the 3-, 5-, 8-, and 10-inch Bonner balls behind each of the mockups and the data $30 \mathrm{~cm}$ behind the $\mathrm{SS}$ and $304.8 \mathrm{~cm}$ from the reactor center are contained in Tables 31 and 27 , respectively. It should be noted that background measurements were made only when the detector was at $304.8 \mathrm{~cm}$.

The next group of slabs added was a combination of aluminum, boron carbide and stainless steel, shown as Item $C$ in Figure 34 . The measurements in Items $A$ and $B$ were repeated behind this mockup and the traverse data with the 5 -inch ball $30 \mathrm{~cm}$ behind the mockup are given in Table 30 and the measurements with the four Bonner balls $13-, 5-, 8-$, and 10-in.) on centerline at $30 \mathrm{~cm}$ and $304.8 \mathrm{~cm}$ are part of Tables 31 and 27. 
For the final mockup, Item VIID, a slab of sodium was added as shown in Figure 34. Traverses with the bare and cd-covered detector along centerline were included as part of the centerline measurements and the data from these detectors and the four $(3-, 5-, 8-$, and 10-in.) Bonner balls are contained in Tables 31 and 27 , bringing to a close the measurements in this experiment. 


\section{ANALYSIS OR EXPERIMENTAL ERRORS}

The errors associated with the measurements are due to a number of uncertainties: (1) the sizes of the gaps between slabs unavoidably introduced in the configurations, (2) in the positions of the detectors, (3) the detector count rate statistics and calibrations, (4) the reactor power determinations, and (5) the effects of the exposure of the configurations to the weather. of these, the uncertainty due to the weather is the least understood and probably beyond simple estimation. The uncertainty lies in the amount of moisture between the slabs and in the lithiated parafin surrounding them. During this experiment the mockups were covered with a plastic tarpaulin that would limit the amount of moisture reaching the slabs. Thus, for this experiment, the effect of the weather was assumed to be negligible.

The TSR-II power level for each measurement was determined from the output of two fission chambers located in the reactor shield along the midplane of the reactor. The response of these chambers to the reactor source was monitored prior to the experiment through the use of gold foils and found to agree to within 5\% with previous reactor power values. These detectors were calibrated on a daily basis using a ${ }^{252} \mathrm{Cf}$ source, with the calibration values lying within about a $6 \%$ spread $( \pm 3 \%$ of an average value). During any one detector traverse in a given day, the variation in the reactor power indicated by the monitor outputs was at most only $3 \%$; however, during the several months the experiment was being performed, the monitors indicated variations of about $\pm 5 \%$. Thus, the uncertainty in the reactor power determination was assumed to be $\pm 5 \%$.

count-rate statistics are expressed in a manner specific to each detector. For the NE-213 measurements, counting statistics and unfolding errors are included in the unfolding of the pulse-height spectra using the FERD code, with the resulting flux expressed in terms of lower and upper limits that represent a $68 \%$ confidence interval. Similar errors are expressed in the tabular data for the hydrogen counter measurements unfolded using SPEC4. Neither of the spectra, NE-213 or hydrogen counter, reflects the error in determining the reactor power since this error is not 
included in the unfolding program. This, as seen above, could be as much as $\pm 5 \%$.

The Bonner balls were calibrated on a daily basis using ${ }^{252} \mathrm{Cf}$ as a source, with the resulting count rates falling within about $\pm 3 \%$ of an average value obtained throughout the years. Movement of the Bonner balls along a traversing mechanism can vary the detector location with respect to the configuration several millimeters on either side of a straight line. For the measurements perpendicular to the configuration centerline at $30 \mathrm{~cm}$ behind the configurations, such variations in the detector position could amount to a change in the count rate of about $2 \%$. For the measurements on centerline beyond the $30 \mathrm{~cm}$ point, the error in positioning several millimeters either side of the selected location would lie within the statistics of the measurement. Rather than calculate probable errors for each measurement in a series of measurements during a traverse, we prefer, in general, to quote a value for the error in the measurements for a given experiment. Thus, assuming the estimated upper limit for all the errors, the errors assigned to the Bonner ball measurements should be less than $\pm 10 \%$. 


\section{REFERENCES}

1. R. E. Maerker et al., Calibration of the Bonner Ball Neutron Detectors Used at the Tower Shielding Facility, ORNL/TM-3465 (June 1971).

2. C. E. Burgart and M. B. Emmett, Monte Carlo Calculations of the Response Functions of Bonner Ball Neutron Detectors, ORNL/TM-3739 (April 1972).

3. B. W. Rust, D. T. Ingersoll, and W. R. Burrus, A User's Manual for the FERDO and FERD Unfolding Codes, ORNL/TM-8720 (September 1983).

4. J. 0. Johnson and D. T. Ingersoll, User's Guide for the Revised SPEC-4 Neutron Spectron Unfolding Code, ORNL/TM-7384 (August 1980).

5. W. W. Engle, Jr., D. T. Ingersoll, C. O. Slater, F. J. Muckenthaler, Specifications for the JASPER Program Attenuation Experiment, ORNL/LMR/AC-86/5 (October 1986).

\section{ACKNOWLEDGEMENTS}

The author is deeply indebted to D. T. Ingersoll and W. W. Engle, Jr. of ORNL's Engineering Physics and Mathematics Division, to D. E. Bartine of the Engineering Technology Division, to P. B. Hemmig of DOE/Washington, and to the JASPER working group from Japan for their participation and assistance in formulating the Experimental Program Plan. Appreciation is expressed to E. R. Specht, Rockwell International, W. H. Harless, General Electric Company, $R$. K. Disney, Westinghouse-ARD, W. L. Bunch, Westinghouse-Hanford for timely suggestions. Special thanks go to G. A. Russell and J. K. Ingersoll for their efforts in editing and preparation of this report. 



\section{APPENDIX A}

\section{Experimental Progran Plan for the JASPER Radial Shield Attenuation Experiment}

I. Spectrum Modifier (SM) for near core shield designs

A. SM1 $(10.27 \mathrm{~cm} \mathrm{Fe}+9.17 \mathrm{~cm} \mathrm{Al}+2.54 \mathrm{~cm}$ Boral $+20.32 \mathrm{~cm}$ Radial Blanket)

1. NE-213 and hydrogen counter measurements on centerline as close as feasible to SM1.

2. 3-, 5-, 10-in Bonner ball on centerline at same location as NE-213.

3. 5-in $B B$ horizontal traverse at $30 \mathrm{~cm}$ behind configuration.

4. 3-, 5-, 8-, 10-in BB measurements on centerline:
a. at $30 \mathrm{~cm}$ behind configuration
b. at $304.8 \mathrm{~cm}$ from reactor centerline (Foreground and Background)
c. at $457.2 \mathrm{~cm}$ from reactor centerline (Foreground and Background).

5. INS ${ }^{3} \mathrm{He} \mathrm{BB}$ measurements on centerline:
a. at $30 \mathrm{~cm}$ behind configuration
b. at $304.8 \mathrm{~cm}$ from reactor centerline (Foreground and Background)
c. at $457.2 \mathrm{~cm}$ from reactor centerline (Foreground and Background).

II. SM + Stainless Steel and $B_{4} C$ combinations (near core shield)

A. $\quad \mathrm{SM} 1+1.27 \mathrm{~cm} \mathrm{Al}+15.24 \mathrm{~cm} \mathrm{SS}$

1. 5-in $\mathrm{BB}$ horizontal traverse at $30 \mathrm{~cm}$ behind configuration.

2. 3-, 5-, 8-, 10-in BB measurements on centerline:
a. at $30 \mathrm{~cm}$ behind configuration
b. at $304.8 \mathrm{~cm}$ from reactor centerline (Foreground and Background) .


B. $\mathrm{SM} 1+1.27 \mathrm{~cm} \mathrm{Al}+15.24 \mathrm{~cm} \mathrm{SS}+1.27 \mathrm{~cm} \mathrm{Al}+15.24 \mathrm{~cm} \mathrm{~B}{ }_{4} \mathrm{C}+$ $2.54 \mathrm{~cm}$ SS

1. 3-, 5-, 8-, 10-in BB measurements on centerline:

a. at $30 \mathrm{~cm}$ behind configuration

b. at $304.8 \mathrm{~cm}$ from reactor centerline (Foreground and Background).

C. $\mathrm{SM1}+1.27 \mathrm{~cm} \mathrm{Al}+15.24 \mathrm{~cm} \mathrm{SS}+1.27 \mathrm{~cm} \mathrm{Al}+15.24 \mathrm{~cm} \mathrm{~B} \mathrm{~B}_{4} \mathrm{C}+$ $2.54 \mathrm{~cm} \mathrm{SS}+1.27 \mathrm{~cm} \mathrm{Al}+10.16 \mathrm{~cm} \mathrm{~B} \mathrm{C}_{4}+5.08 \mathrm{~cm} \mathrm{~B} \mathrm{~B}_{4} \mathrm{C}$

1. 3-, 5-, 8-, 10-in BB measurements on centerline:

a. at $30 \mathrm{~cm}$ behind configuration

b. at $304.8 \mathrm{~cm}$ from reactor centerline (Foreground and Background).

D. $\quad \mathrm{SM} 1+1.27 \mathrm{~cm} \mathrm{Al}+15.24 \mathrm{~cm} \mathrm{SS}+1.27 \mathrm{~cm} \mathrm{Al}+15.24 \mathrm{~cm} \mathrm{~B} \mathrm{~B}_{4} \mathrm{C}+$ $2.54 \mathrm{~cm} \mathrm{SS}+1.27 \mathrm{~cm} \mathrm{Al}+10.16 \mathrm{~cm} \mathrm{~B} \mathrm{~B}_{4} \mathrm{C}+5.08 \mathrm{~cm} \mathrm{~B} \mathrm{~B}_{4}+1.27 \mathrm{~cm} \mathrm{Al}+$ $10.16 \mathrm{~cm} \mathrm{~B} \mathrm{~B}_{4} \mathrm{C}+5.08 \mathrm{~cm} \mathrm{~B} \mathrm{~B}_{4} \mathrm{C}$

1. NE-213 and hydrogen counter measurements on centerline as close as feasible behind configuration.

2. 3-, 5-, 10-in BB measurements on centerline at same location as NE-213.

3. 5-in BB horizontal traverse at $30 \mathrm{~cm}$ behind configuration.

4. 3-, 5-, 8-, 10-in BB measurements on centerline:
a. at $30 \mathrm{~cm}$ behind configuration
b. at $304.8 \mathrm{~cm}$ from reactor centerline (Foreground and Background).

5. INS ${ }^{3} \mathrm{He} \mathrm{BB}$ measurements on centerline:
a. at $30 \mathrm{~cm}$ behind configuration
b. at $304.8 \mathrm{~cm}$ from reactor centerline (Foreground and Background).

E. $\quad \mathrm{SM} 1+1.27 \mathrm{~cm} \mathrm{Al}+15.24 \mathrm{~cm} \mathrm{SS}+1.27 \mathrm{~cm} \mathrm{Al}+15.24 \mathrm{~cm} \mathrm{~B} \mathrm{~B}_{4} \mathrm{C}+$ $2.54 \mathrm{~cm} \mathrm{SS}+1.27 \mathrm{~cm} \mathrm{Al}+10.16 \mathrm{~cm} \mathrm{~B}{ }_{4} \mathrm{C}+5.08 \mathrm{~cm} \mathrm{~B} \mathrm{C}_{4} \mathrm{C}+1.27 \mathrm{~cm} \mathrm{Al}+$ $10.16 \mathrm{~cm} \mathrm{~B}{ }_{4} \mathrm{C}+5.08 \mathrm{~cm} \mathrm{~B}{ }_{4} \mathrm{C}+30.48 \mathrm{~cm} \mathrm{Na}$

1. 5-in $\mathrm{BB}$ horizontal traverse at $30 \mathrm{~cm}$ behind configuration. 
2. Bare, Cd-covered, 3-, 5-, 8-, 10-in BB measurements on centerline:
a. at $30 \mathrm{~cm}$ behind configuration
b. at $304.8 \mathrm{~cm}$ from reactor centerline (Foreground and Background).

3. Bare detector on centerline at $30 \mathrm{~cm}$ behind configuration inside partial cadmium enclosure that surrounded the detector except on the side of the last slab in the configuration.

4. Bare detector on centerline at $501.3 \mathrm{~cm}$ point with cadmium sheet over face of last slab in the configuration.

III. $S M+S S$ and $B_{4} C$

A. $S M 1+10.16 \mathrm{~cm} \mathrm{SS}$

1. 5-in BB horizontal traverse at $30 \mathrm{~cm}$ behind configuration.

2. 3-, 5-, 8-, 10-in BB measurements on centerline:
a. at $30 \mathrm{~cm}$ behind configuration
b. at $304.8 \mathrm{~cm}$ from reactor centerline (Foreground and Background).

B. $\quad \mathrm{SM} 1+10.16 \mathrm{~cm} \mathrm{SS}+5.08 \mathrm{~cm} \mathrm{~B} \mathrm{~B}_{4} \mathrm{C}$

1. 5-in BB horizontal traverse at $30 \mathrm{~cm}$ behind configuration.

2. 3-, 5-, 8-, 10-in BB measurements on centerline:
a. at $30 \mathrm{~cm}$ behind configuration
b. at $304.8 \mathrm{~cm}$ from reactor centerline (Foreground and Background).

C. $\mathrm{SM} 1+20.32 \mathrm{~cm} \mathrm{SS}$

1. 5-in BB horizontal traverse at $30 \mathrm{~cm}$ behind configuration.

2. 3-, 5-, 8-, 10-in BB measurements on centerline:
a. at $30 \mathrm{~cm}$ behind configuration
b. at $304.8 \mathrm{~cm}$ from reactor centerline (Foreground and Background).


D. $\quad \mathrm{SM} 1+20.32 \mathrm{~cm} \mathrm{SS}+5.08 \mathrm{~cm} \mathrm{~B} \mathrm{~B}_{4} \mathrm{C}$

1. 3-, 5-, 8-, 10-in BB measurements on centerline:

a. at $30 \mathrm{~cm}$ behind configuration

b. at $304.8 \mathrm{~cm}$ from reactor centerline (Foreground and Background).

E. $\quad \mathrm{SM} 1+20.32 \mathrm{~cm} \mathrm{SS}+5.08 \mathrm{~cm} \mathrm{~B} \mathrm{~B}_{4} \mathrm{C}+5.08 \mathrm{~cm} \mathrm{~B} \mathrm{~B}_{4} \mathrm{C}$

1. 5 -in $B B$ horizontal traverse at $30 \mathrm{~cm}$ behind configuration.

2. 3-, 5-, 8-, 10-in BB measurements on centerline:

a. at $30 \mathrm{~cm}$ behind configuration

b. at $304.8 \mathrm{~cm}$ from reactor centerline (Foreground and Background).

IV. $S M+$ Graphite and $B_{4} C$ (tank type shield reference)

A. $\quad \mathrm{SM1}+1.27 \mathrm{~cm} \mathrm{Al}+15.24 \mathrm{~cm} \mathrm{SS}+1.27 \mathrm{~cm} \mathrm{Al}+10.16 \mathrm{~cm} \mathrm{C}+5.08 \mathrm{~cm}$ SS

1. 5-in BB horizontal traverse at $30 \mathrm{~cm}$ behind configuration

2. 3-, 5-, 8-, 10-in BB measurements on centerline:

a. at $30 \mathrm{~cm}$ behind configuration

b. at $304.8 \mathrm{~cm}$ from reactor centerline (Foreground and Background ) .

B. $\quad \mathrm{SM1}+1.27 \mathrm{~cm} \mathrm{Al}+15.24 \mathrm{~cm} \mathrm{SS}+1.27 \mathrm{~cm} \mathrm{Al}+10.16 \mathrm{~cm} \mathrm{C}+5.08 \mathrm{~cm}$ $\mathrm{SS}+1.27 \mathrm{~cm} \mathrm{Al}+10.16 \mathrm{~cm} \mathrm{C} \div 5.08 \mathrm{~cm} \mathrm{SS}$

1. $3-, 5-, 8-, 10-$ in $B B$ measurements on centerline:

a. at $30 \mathrm{~cm}$ behind configuration

b. at $304.8 \mathrm{~cm}$ from reactor centerline (Foreground and Background).

C. $\quad \mathrm{SMI}+1.27 \mathrm{~cm} \mathrm{Al}+15.24 \mathrm{~cm} \mathrm{SS}+1.27 \mathrm{~cm} \mathrm{Al}+10.16 \mathrm{~cm} \mathrm{C}+5.08 \mathrm{~cm}$ $\mathrm{SS}+1.27 \mathrm{~cm} \mathrm{Al}+10.16 \mathrm{~cm} \mathrm{C}+5.08 \mathrm{~cm} \mathrm{SS}+1.27 \mathrm{~cm} \mathrm{Al}+15.24 \mathrm{~cm}$ $\mathrm{B}_{4} \mathrm{C}+2.54 \mathrm{~cm} \mathrm{SS}$

1. Ne-213 and hydrogen counter measurements on centerline as close as feasible behind ss. 
2. 3-, 5-, 10-in BB measurements on centerline at same location as NE-213.

3. 5-in $B B$ horizontal traverse at $30 \mathrm{~cm}$ behind configuration.

4. 3-, 5-, 8-, 10-in BB measurements on centerline:
a. at $30 \mathrm{~cm}$ behind configuration
b. at $304.8 \mathrm{~cm}$ from reactor centerline (Foreground and Background)
c. at $457.2 \mathrm{~cm}$ from reactor centerline (Foreground and Background).

5. INS ${ }^{3} \mathrm{He}$ BB measurements on centerline:
a. at $30 \mathrm{~cm}$ behind configuration
b. at $304.8 \mathrm{~cm}$ from reactor centerline (Foreground and Background)
c. at $457.2 \mathrm{~cm}$ from reactor centerline (Foreground and Background).

D. $\quad \mathrm{SM} 1+1.27 \mathrm{~cm} \mathrm{Al}+15.24 \mathrm{~cm} \mathrm{SS}+1.27 \mathrm{~cm} \mathrm{Al}+10.16 \mathrm{~cm} \mathrm{C}+5.08 \mathrm{~cm}$ $\mathrm{SS}+1.27 \mathrm{~cm} \mathrm{Al}+10.16 \mathrm{~cm} \mathrm{C}+5.08 \mathrm{~cm} \mathrm{SS}+1.27 \mathrm{~cm} \mathrm{Al}+15.24 \mathrm{~cm}$ $\mathrm{B}_{4} \mathrm{C}+2.54 \mathrm{~cm} \mathrm{SS}+2.54 \mathrm{~cm} \mathrm{Al}+5.08 \mathrm{~cm} \mathrm{SS}$

1. 3-, 5-, 8-, 10-in BB measurements on centerline:
a. at $30 \mathrm{~cm}$ behind configuration
b. at $457.2 \mathrm{~cm}$ from reactor centerline (Foreground and Background).

E. $\mathrm{SM} 1+1.27 \mathrm{~cm} \mathrm{Al}+15.24 \mathrm{~cm} \mathrm{SS}+1.27 \mathrm{~cm} \mathrm{Al}+10.16 \mathrm{~cm} \mathrm{C}+5.08 \mathrm{~cm}$ $\mathrm{SS}+1.27 \mathrm{~cm} \mathrm{Al}+10.16 \mathrm{~cm} \mathrm{C}+5.08 \mathrm{~cm} \mathrm{SS}+1.27 \mathrm{~cm} \mathrm{Al}+15.24 \mathrm{~cm}$ $\mathrm{B}_{4} \mathrm{C}+2.54 \mathrm{~cm} \mathrm{SS}+2.54 \mathrm{~cm} \mathrm{Al}+5.08 \mathrm{~cm} \mathrm{SS}+1.27 \mathrm{~cm} \mathrm{Al}+$ $10.16 \mathrm{~cm} \mathrm{C}+5.08 \mathrm{~cm} \mathrm{SS}$

1. 3-, 5-, 8-, 10-in BB measurements on centerline:
a. at $30 \mathrm{~cm}$ behind configuration
b. at $457.2 \mathrm{~cm}$ from reactor centerline (Foreground and Background).

F. $\quad \mathrm{SM} 1+1.27 \mathrm{~cm} \mathrm{Al}+15.24 \mathrm{~cm} \mathrm{SS}+1.27 \mathrm{~cm} \mathrm{Al}+10.16 \mathrm{~cm} \mathrm{C}+5.08 \mathrm{~cm}$ $\mathrm{SS}+1.27 \mathrm{~cm} \mathrm{Al}+10.16 \mathrm{~cm} \mathrm{C}+5.08 \mathrm{~cm} \mathrm{SS}+1.27 \mathrm{~cm} \mathrm{Al}+15.24 \mathrm{~cm}$ $\mathrm{B}_{4} \mathrm{C}+2.54 \mathrm{~cm} \mathrm{SS}+2.54 \mathrm{~cm} \mathrm{Al}+5.08 \mathrm{~cm} \mathrm{SS}+1.27 \mathrm{~cm} \mathrm{Al}+$ $10.16 \mathrm{~cm} \mathrm{C}+5.08 \mathrm{~cm} \mathrm{SS}+1.27 \mathrm{~cm} \mathrm{Al}+10.16 \mathrm{~cm} \mathrm{C}+5.08 \mathrm{~cm} \mathrm{SS}$ 
1. 3-, 5-, 8-, 10-in BB measurements on centerline:

a. at $30 \mathrm{~cm}$ behind configuration

b. at $457.2 \mathrm{~cm}$ from reactor centerline (Foreground and Background).

2. 3-, 5-, 8-, 10-in BB measurements on centerline as close as feasible:

a. Foreground (same location as background)

b. Background $(30.48 \mathrm{~cm} \mathrm{LiH} \mathrm{between} \mathrm{detector} \mathrm{and}$ configuration).

G. $\quad \mathrm{SM} 1+1.27 \mathrm{~cm} \mathrm{Al}+15.24 \mathrm{~cm} \mathrm{SS}+1.27 \mathrm{~cm} \mathrm{Al}+10.16 \mathrm{~cm} \mathrm{C}+5.08 \mathrm{~cm}$ $\mathrm{SS}+1.27 \mathrm{~cm} \mathrm{Al}+10.16 \mathrm{~cm} \mathrm{C}+5.08 \mathrm{~cm} \mathrm{SS}+1.27 \mathrm{~cm} \mathrm{Al}+15.24 \mathrm{~cm}$ $\mathrm{B}_{4} \mathrm{C}+2.54 \mathrm{~cm} \mathrm{SS}+2.54 \mathrm{~cm} \mathrm{Al}+5.08 \mathrm{~cm} \mathrm{SS}+1.27 \mathrm{~cm} \mathrm{Al}+10.16 \mathrm{~cm}$ $\mathrm{C}+5.08 \mathrm{~cm} \mathrm{SS}+1.27 \mathrm{~cm} \mathrm{Al}+10.16 \mathrm{~cm} \mathrm{C}+5.08 \mathrm{~cm} \mathrm{SS}+1.27 \mathrm{~cm} \mathrm{Al}$ $+10.16 \mathrm{~cm} \mathrm{C}+5.08 \mathrm{~cm} \mathrm{SS}$

1. 3-, 5-, 8-, 10-in BB measurements on centerline as close as feasible:

a. Foreground (same location as for background)

b. Background $(30.48 \mathrm{~cm} \mathrm{LiH} \mathrm{between} \mathrm{detector} \mathrm{and}$ configuration).

2. INS ${ }^{3} \mathrm{He} \mathrm{BB}$ measurements on centerline as close as feasible:

a. Foreground (same location as for background)

b. Background $(30.48 \mathrm{~cm} \mathrm{LiH} \mathrm{between} \mathrm{detector} \mathrm{and}$ configuration).

H. $\quad \mathrm{SM} 1+1.27 \mathrm{~cm} \mathrm{Al}+15.24 \mathrm{~cm} \mathrm{SS}+1.27 \mathrm{~cm} \mathrm{Al}+10.16 \mathrm{~cm} \mathrm{C}+5.08 \mathrm{~cm}$ $\mathrm{SS}+1.27 \mathrm{~cm} \mathrm{Al}+10.16 \mathrm{~cm} \mathrm{C}+5.08 \mathrm{~cm} \mathrm{SS}+1.27 \mathrm{~cm} \mathrm{Al}+15.24 \mathrm{~cm}$ $\mathrm{B}_{4} \mathrm{C}+2.54 \mathrm{~cm} \mathrm{SS}+2.54 \mathrm{~cm} \mathrm{Al}+5.08 \mathrm{~cm} \mathrm{SS}+1.27 \mathrm{~cm} \mathrm{Al}+10.16 \mathrm{~cm}$ $\mathrm{C}+5.08 \mathrm{~cm} \mathrm{SS}+1.27 \mathrm{~cm} \mathrm{Al}+10.16 \mathrm{~cm} \mathrm{C}+5.08 \mathrm{~cm} \mathrm{SS}+1.27 \mathrm{~cm} \mathrm{Al}$ $+10.16 \mathrm{~cm} \mathrm{C}+5.08 \mathrm{~cm} \mathrm{SS}+5.08 \mathrm{~cm} \mathrm{SS}$

1. 3-, 5-, 8-, 10-in BB measurements on centerline as close as feasible:

a. Foreground (same location as for background)

b. Background $(30.48 \mathrm{~cm} \mathrm{LiH} \mathrm{between} \mathrm{detector} \mathrm{and}$ configuration). 
I. $\mathrm{SM} 1+1.27 \mathrm{~cm} \mathrm{Al}+15.24 \mathrm{~cm} \mathrm{SS}+1.27 \mathrm{~cm} \mathrm{Al}+10.16 \mathrm{~cm} \mathrm{C}+5.08 \mathrm{~cm}$ $\mathrm{SS}+1.27 \mathrm{~cm} \mathrm{Al}+10.16 \mathrm{~cm} \mathrm{C}+5.08 \mathrm{~cm} \mathrm{SS}+1.27 \mathrm{~cm} \mathrm{Al}+15.24 \mathrm{~cm}$ $\mathrm{B}_{4} \mathrm{C}+2.54 \mathrm{~cm} \mathrm{SS}+2.54 \mathrm{~cm} \mathrm{Al}+5.08 \mathrm{~cm} \mathrm{SS}+1.27 \mathrm{~cm} \mathrm{Al}+10.16 \mathrm{~cm}$ $\mathrm{C}+5.08 \mathrm{~cm} \mathrm{SS}+1.27 \mathrm{~cm} \mathrm{Al}+10.16 \mathrm{~cm} \mathrm{C}+5.08 \mathrm{~cm} \mathrm{SS}+1.27 \mathrm{~cm} \mathrm{Al}$ $+10.16 \mathrm{~cm} \mathrm{C}+5.08 \mathrm{~cm} \mathrm{SS}+5.08 \mathrm{~cm} \mathrm{SS}+30.48 \mathrm{~cm} \mathrm{Na}$

1. bare, cd-covered, 3-, 5-, 8-, 10-in BB measurements on centerline as close as feasible:
a. Foreground (same as for background)
b. Background $(30.48 \mathrm{~cm} \mathrm{LiH} \mathrm{between} \mathrm{detector} \mathrm{and}$ configuration).

IA. $\quad \mathrm{SM} 1+1.27 \mathrm{~cm} \mathrm{Al}+15.24 \mathrm{~cm} \mathrm{SS}+1.27 \mathrm{~cm} \mathrm{Al}+10.16 \mathrm{~cm} \mathrm{C}-5.08 \mathrm{~cm}$ $\mathrm{SS}+1.27 \mathrm{~cm} \mathrm{Al}+10.16 \mathrm{~cm} \mathrm{C}+5.08 \mathrm{~cm} \mathrm{SS}+1.27 \mathrm{~cm} \mathrm{Al}+5.24 \mathrm{~cm}$ $\mathrm{B}_{4} \mathrm{C}+2.54 \mathrm{~cm} \mathrm{SS}+2.54 \mathrm{~cm} \mathrm{Al}+5.08 \mathrm{~cm} \mathrm{SS}+1.27 \mathrm{~cm} \mathrm{Al}+10.16 \mathrm{~cm}$ $\mathrm{C}^{4}+5.08 \mathrm{~cm} \mathrm{SS}+1.27 \mathrm{~cm} \mathrm{Al}+10.16 \mathrm{~cm} \mathrm{C}+5.08 \mathrm{~cm} \mathrm{SS}+1.27 \mathrm{~cm} \mathrm{Al}$ $+10.16 \mathrm{~cm} \mathrm{C}+5.08 \mathrm{~cm} \mathrm{SS}+5.08 \mathrm{~cm} \mathrm{SS}+30.48 \mathrm{~cm} \mathrm{Na}+30 \mathrm{~cm}$ Void + $30.48 \mathrm{~cm} \mathrm{Na}$

1. bare, cd-covered, 3-, 5-, 8-, 10-in BB on centerljne in $30 \mathrm{~cm}$ void.

J. $\quad \mathrm{SM} 1+1.27 \mathrm{~cm} \mathrm{Al}+15.24 \mathrm{~cm} \mathrm{SS}+1.27 \mathrm{~cm} \mathrm{Al}+10.16 \mathrm{~cm} \mathrm{C}+5.08 \mathrm{~cm} \mathrm{SS}+$ $1.27 \mathrm{~cm} \mathrm{Al}+10.16 \mathrm{~cm} \mathrm{C}+5.08 \mathrm{~cm} \mathrm{SS}+1.27 \mathrm{~cm} \mathrm{Al}+15.24 \mathrm{~cm} \mathrm{~B} \mathrm{~B}_{4}+$ $2.54 \mathrm{~cm} \mathrm{SS}+2.54 \mathrm{~cm} \mathrm{Al}+5.08 \mathrm{~cm} \mathrm{SS}+1.27 \mathrm{~cm} \mathrm{Al}+10.16 \mathrm{~cm} \mathrm{C}+5.08 \mathrm{~cm}$ $\mathrm{SS}+1.27 \mathrm{~cm} \mathrm{Al}+10.16 \mathrm{~cm} \mathrm{C}+5.08 \mathrm{~cm} \mathrm{SS}+1.27 \mathrm{~cm} \mathrm{Al}+10.16 \mathrm{~cm} \mathrm{C}+$ $5.08 \mathrm{~cm} \mathrm{SS}+5.08 \mathrm{~cm} \mathrm{SS}+61 \mathrm{~cm} \mathrm{Na}+30 \mathrm{~cm} \mathrm{Void}+30.48 \mathrm{~cm} \mathrm{Na}$

1. bare, cd-covered, 3-, 5-, 8-, 10-in BB on centerline in $30 \mathrm{~cm}$ void.

2. INS ${ }^{3} \mathrm{He} \mathrm{BB}$ on centerline in $30 \mathrm{~cm}$ void.

K. $\quad \mathrm{SM} 1+1.27 \mathrm{~cm} \mathrm{Al}+15.24 \mathrm{~cm} \mathrm{SS}+1.27 \mathrm{~cm} \mathrm{Al}+10.16 \mathrm{~cm} \mathrm{C}+5.08 \mathrm{~cm} \mathrm{SS}+$ $1.27 \mathrm{~cm} \mathrm{Al}+10.16 \mathrm{~cm} \mathrm{C}+5.08 \mathrm{~cm} \mathrm{SS}+1.27 \mathrm{~cm} \mathrm{Al}+15.24 \mathrm{~cm} \mathrm{~B} \mathrm{~B}_{4} \mathrm{C}+$ $2.54 \mathrm{~cm} \mathrm{SS}+2.54 \mathrm{~cm} \mathrm{Al}+5.08 \mathrm{~cm} \mathrm{SS}+1.27 \mathrm{~cm} \mathrm{Al}+10.16 \mathrm{~cm} \mathrm{C}+5.08 \mathrm{~cm}$ $\mathrm{SS}+1.27 \mathrm{~cm} \mathrm{Al}+10.16 \mathrm{~cm} \mathrm{C} \div 5.08 \mathrm{~cm} \mathrm{SS}+1.27 \mathrm{~cm} \mathrm{Al}+10.16 \mathrm{~cm} \mathrm{C}+$ $5.08 \mathrm{~cm} \mathrm{SS}+5.08 \mathrm{~cm} \mathrm{SS}+91 \mathrm{~cm} \mathrm{Na}+30 \mathrm{~cm} \mathrm{Void}+30.48 \mathrm{~cm} \mathrm{Na}$

1. bare, cd-covered, 3-, 5-, 8-, 10-in $B B$ on centerline in $30 \mathrm{~cm}$ void.

L. $\quad \mathrm{SM} 1+1.27 \mathrm{~cm} \mathrm{Al}+15.24 \mathrm{~cm} \mathrm{SS}+1.27 \mathrm{~cm} \mathrm{Al}+10.16 \mathrm{~cm} \mathrm{C}+5.08 \mathrm{~cm} \mathrm{SS}+$ $1.27 \mathrm{~cm} \mathrm{Al}+10.16 \mathrm{~cm} \mathrm{C}+5.08 \mathrm{~cm} \mathrm{SS}+1.27 \mathrm{~cm} \mathrm{Al}+15.24 \mathrm{~cm} \mathrm{~B} \mathrm{C}_{4}+$ $2.54 \mathrm{~cm} \mathrm{SS}+2.54 \mathrm{~cm} \mathrm{Al}+5.08 \mathrm{~cm} \mathrm{SS}+1.27 \mathrm{~cm} \mathrm{Al}+10.16 \mathrm{~cm} \mathrm{C}+5.08 \mathrm{~cm}$ $\mathrm{SS}+1.27 \mathrm{~cm} \mathrm{Al}+10.16 \mathrm{~cm} \mathrm{C}+5.08 \mathrm{~cm} \mathrm{SS}+1.27 \mathrm{~cm} \mathrm{Al}+10.16 \mathrm{~cm} \mathrm{C}+$ $5.08 \mathrm{~cm} \mathrm{SS}+5.08 \mathrm{~cm} \mathrm{SS}+122 \mathrm{~cm} \mathrm{Na}+30 \mathrm{~cm} \mathrm{Void}+30.48 \mathrm{~cm} \mathrm{Na}$

1. bare, cd-covered, 3-, 5-, 8-, 10-in BB on centeriine in $30 \mathrm{~cm}$ void. 
M. $\quad \mathrm{SM} 1+1.27 \mathrm{~cm} \mathrm{Al}+15.24 \mathrm{~cm} \mathrm{SS}+1.27 \mathrm{~cm} \mathrm{Al}+10.16 \mathrm{~cm} \mathrm{C}+5.08 \mathrm{~cm} \mathrm{SS}+$ $1.27 \mathrm{~cm} \mathrm{Al}+10.16 \mathrm{~cm} \mathrm{C}+5.08 \mathrm{~cm} \mathrm{SS}+1.27 \mathrm{~cm} \mathrm{Al}+15.24 \mathrm{~cm} \mathrm{~B} 4+$ $2.54 \mathrm{~cm} \mathrm{SS}+2.54 \mathrm{~cm} \mathrm{Al}+5.08 \mathrm{~cm} \mathrm{SS}+1.27 \mathrm{~cm} \mathrm{Al}+10.16 \mathrm{~cm} \mathrm{C}+5.08 \mathrm{~cm}$ $\mathrm{SS}+1.27 \mathrm{~cm} \mathrm{Al}+10.16 \mathrm{~cm} \mathrm{C}+5.08 \mathrm{~cm} \mathrm{SS}+1.27 \mathrm{~cm} \mathrm{Al}+10.16 \mathrm{~cm} \mathrm{C}+$ $5.08 \mathrm{~cm} \mathrm{SS}+5.08 \mathrm{~cm} \mathrm{SS}+152.4 \mathrm{~cm} \mathrm{Na}+30 \mathrm{~cm} \mathrm{Void}+30.48 \mathrm{~cm} \mathrm{Na}$

1. bare, cd-covered, 3-, 5-, 8-, 10-in $B B$ on centerline in $30 \mathrm{~cm}$ void.

2. INS ${ }^{3} \mathrm{He} \mathrm{BB}$ on centerline in $30 \mathrm{~cm}$ void.

V. $S M+S S+B_{4} C($ IHX Shield)

A. SM2 $(10.27 \mathrm{~cm} \mathrm{Fe}+9.17 \mathrm{~cm} \mathrm{Al}+2.54 \mathrm{~cm} \mathrm{Boral}+182.88 \mathrm{~cm} \mathrm{Na})$

1. 5-in $B B$ horizontal traverse through midplane at $30 \mathrm{~cm}$ behind configuration.

2. Bare, cd-covered, 3-, 5-, 8-, 10-in BB measurements on centerline:

a. at $30 \mathrm{~cm}$ behind configuration

b. at $501.3 \mathrm{~cm}$ from reactor centerline (Foreground and Background).

3. Bare detector on centerline at $30 \mathrm{~cm}$ behind configuration inside partial cadmium enclosure that surrounded the detector except on the side of the last slab in the configuration.

4. Bare detector on centerline at $501.3 \mathrm{~cm}$ point with cadmium sheet over face of last slab in the configuration.

5. INS ${ }^{3} \mathrm{He} \mathrm{BB}$ measurements on centerline:

a. at $30 \mathrm{~cm}$ behind configuration

b. at $501.3 \mathrm{~cm}$ from reactor centerline (Foreground and Background).

B. $\quad \mathrm{SM} 2+5.08 \mathrm{~cm} \mathrm{SS}$

1. 5-in BB horizontal traverse through midplane at $30 \mathrm{~cm}$ behind configuration.

2. Bare, cd-covered, 3-, 5-, 8-, 10-in BB measurements on centerline:

a. at $30 \mathrm{~cm}$ behind configuration 
b. at $501.3 \mathrm{~cm}$ from reactor centerline (Foreground and
Background).

3. Bare detector on centerline at $30 \mathrm{~cm}$ behind configuration inside partial cadmium enclosure that surrounded the detector except on the side of the last slab in the configuration.

4. Bare detector on centerline at $501.3 \mathrm{~cm}$ point with cadmium sheet over face of last slab in the configuration.

C. $\mathrm{SM} 2+5.08 \mathrm{~cm} \mathrm{SS}+30.48 \mathrm{~cm} \mathrm{Na}$

1. Bare, cd-covered, 3-, 5-, 8-, 10--in BB measurements on centerline:
a. at $30 \mathrm{~cm}$ behind conf iguration
b. at $501.3 \mathrm{~cm}$ from reactor centerline (Foreground and Background).

2. Bare detector on centerline at $30 \mathrm{~cm}$ behind configuration inside partial cadmium enclosure that surrounded the detector except on the side of the last slab in the configuration.

3. Bare detector on centerline at $501.3 \mathrm{~cm}$ point with cadmium sheet over face of last slab in the configuration.

D. $\mathrm{SM} 2+5.08 \mathrm{~cm} \mathrm{SS}+61 \mathrm{~cm} \mathrm{Na}$

1. 5-in BB horizontal traverse through midplane at $30 \mathrm{~cm}$ behind configuration.

2. Bare, cd-covered, 3-, 5-, 8-, 10-in BB measurements on centerline:
a. at $30 \mathrm{~cm}$ behind configuration
b. at $501.3 \mathrm{~cm}$ from reactor centerline (Foreground and Background).

3. Bare detector on centerline at $30 \mathrm{~cm}$ behind configuration inside partial cadmium enclosure that surrounded the detector except on the side of the last slab in the configuration.

4. Bare detector on centerline at $501.3 \mathrm{~cm}$ point with cadmium sheet over face of last slab in the configuration.

5. INS ${ }^{3}$ He BB measurements on centerline:

a. at $30 \mathrm{~cm}$ behind configuration 
b. at $501.3 \mathrm{~cm}$ from reactor centerline (Foreground and Background).

E. $\quad \mathrm{SM} 2+5.08 \mathrm{~cm} \mathrm{~B} \mathrm{~B}_{4} \mathrm{C}+5.08 \mathrm{~cm} \mathrm{SS}$

1. Bare, cd-covered, 3-, 5-, 8-, 10-in BB measurements on centerline:
a. at $30 \mathrm{~cm}$ behind configuration
b. at $501.3 \mathrm{~cm}$ from reactor centerline (Foreground and Background).

2. Bare detector on centerline at $30 \mathrm{~cm}$ behind configuration inside partial cadmium enclosure that surrounded the detector except on the side of the last slab in the configuration.

3. Bare detector on centerline at $501.3 \mathrm{~cm}$ point with cadmium sheet over face of last slab in the configuration.

F. $\quad \mathrm{SM} 2+5.08 \mathrm{~cm} \mathrm{~B}{ }_{4} \mathrm{C}+5.08 \mathrm{~cm} \mathrm{SS}+30.48 \mathrm{~cm} \mathrm{Na}$

1. Bare, cd-covered, 3-, 5-, 8-, 10-in BB measurements on centerline:
a. at $30 \mathrm{~cm}$ behind configuration
b. at $501.3 \mathrm{~cm}$ from reactor centerline (Foreground and Background).

2. Bare detector on centerline at $30 \mathrm{~cm}$ behind configuration inside partial cadmium enclosure that surrounded the detector except on the side of the last slab in the configuration.

3. Bare detector on centerline at $501.3 \mathrm{~cm}$ point with cadmium sheet over face of last slab in the configuration.

G. $\quad \mathrm{SM} 2+5.08 \mathrm{~cm} \mathrm{~B} \mathrm{~m}_{4} \mathrm{C}+5.08 \mathrm{~cm} \mathrm{SS}+61 \mathrm{~cm} \mathrm{Na}$

1. 5-in $\mathrm{BB}$ horizontal traverse through midplane at $30 \mathrm{~cm}$ behind configuration.

2. Bare, cd-covered, 3-, 5-, 8-, 10-in BB measurements on centerline:
a. at $30 \mathrm{~cm}$ behind configuration
b. at $501.3 \mathrm{~cm}$ from reactor center]ine (Foreground and Background).


3. Bare detector on centerline at $30 \mathrm{~cm}$ behind configuration inside partial cadmium enclosure that surrounded the detector except on the side of the last slab in the configuration.

4. Bare detector on centerline at $501.3 \mathrm{~cm}$ point with cadmium sheet over face of last slab in the configuration.

5. INS ${ }^{3} \mathrm{He} \mathrm{BB}$ measurements on centerline:

a. at $30 \mathrm{~cm}$ behind configuration

b. at $501.3 \mathrm{~cm}$ from reactor centerline (Foreground and Background).

H. $\mathrm{SM} 2+10.16 \mathrm{~cm} \mathrm{~B} 4 \mathrm{C}+5.08 \mathrm{~cm} \mathrm{SS}$

1. Bare, cd-covered, 3-, 5-, 8-, 10-in BB measurements on centerline:
a. at $30 \mathrm{~cm}$ behind configuration b. at $501.3 \mathrm{~cm}$ from reactor centerline (Foreground and
Background).

2. Bare detector on centerline at $30 \mathrm{~cm}$ behind configuration inside partial cadmium enclosure that surrounded the detector except on the side of the last slab in the configuration.

3. Bare detector on centerline at $501.3 \mathrm{~cm}$ point with cadmium sheet over face of last slab in the configuration.

I. $\mathrm{SM2}+10.16 \mathrm{~cm} \mathrm{~B} \mathrm{~B}_{4} \mathrm{C}+5.08 \mathrm{~cm} \mathrm{SS}+30.48 \mathrm{~cm} \mathrm{Na}$

1. Bare, cd-covered, 3-, 5-, 8-, 10-in BB measurements on centerline:
a. at $30 \mathrm{~cm}$ behind configuration
b. at $501.3 \mathrm{~cm}$ from reactor centerline (Foreground and Background).

2. Bare detector on centerline at $30 \mathrm{~cm}$ behind configuration inside partial cadmium enclosure that surrounded the detector except on the side of the last slab in the configuration.

3. Bare detector on centerline at $501,3 \mathrm{~cm}$ point with cadmium sheet over face of last slab in the configuration.

J. $\quad S M 2 \div 10.16 \mathrm{~cm} \mathrm{~B}{ }_{4} C+5.08 \mathrm{~cm} \mathrm{SS}+61 \mathrm{~cm} \mathrm{Na}$

1. 5-in $\mathrm{BB}$ horizontal traverse through midplane at $30 \mathrm{~cm}$ behind configuration. 
2. Bare, cd-covered, 3-, 5-, 8-, 10-in BB measurements on centerline :
a. at $30 \mathrm{~cm}$ behind configuration
b. at $501.3 \mathrm{~cm}$ from reactor centerline (Foreground and Background ) .

3. Bare detector on centerline at $30 \mathrm{~cm}$ behind configuration inside partial cadmium enclosure that surrounded the detector except on the side of the last slab in the configuration.

4. Bare detector on centerline at $501.3 \mathrm{~cm}$ point with cadmium sheet over face of last slab in the configuration.

5. INS ${ }^{3} \mathrm{He}$ BB measurements on centerline:
a. at $30 \mathrm{~cm}$ behind configuration
b. at $501.3 \mathrm{~cm}$ from reactor centerline (Foreground and Background I.

VI. SM + SS and Graphite (Graphite benchmark)

A. SM1 $(10.27 \mathrm{~cm} \mathrm{Fe}+9.17 \mathrm{~cm} \mathrm{Al}+2.54 \mathrm{~cm}$ boral $+20.32 \mathrm{~cm}$ radial

blanket) $\div 1.27 \mathrm{~cm} \mathrm{Al} \div 15.24 \mathrm{~cm} \mathrm{SS}+10.16 \mathrm{~cm}$ graphite

1. NE-213 and hydrogen counter measurements on centerline as close as feasible behind SS.

2. 3-, 5-, 10-in BB measurements on centerline at same location as $\mathrm{NE}-213$.

3. 5-in $\mathrm{BB}$ horizontal traverse at $30 \mathrm{~cm}$ behind configuration.

4. Bare, cd-covered, 3-, 5-, 8-, 10-in BB on centerline:
a. at $30 \mathrm{~cm}$ behind configuration
b. at $304.8 \mathrm{~cm}$ from reactor centerline (Foreground and Background).

5. Bare detector on centerline at $30 \mathrm{~cm}$ behind configuration inside partial cadmium enclosure that surrounded the detector except on the side of the last slab in the configuration.

6. Bare detector on centerline at $304.8 \mathrm{~cm}$ point with cadmium sheet over face of last slab in the configuration.

7. INS ${ }^{3}$ He $\mathrm{BB}$ measurements on centerline: 
a. at $30 \mathrm{~cm}$ behind configuration
b. at $304.8 \mathrm{~cm}$ from reactor centerline (Foreground and
Background).

B. $\quad \mathrm{SM1}+1.27 \mathrm{~cm} \mathrm{Al}+15.24 \mathrm{~cm} \mathrm{SS}+20.32 \mathrm{~cm}$ graphite

1. 5-in $B B$ horizontal traverse at $30 \mathrm{~cm}$ behind configuration.

2. Bare, cd-covered, 3-, 5-, 8-, 10-in BB on centerline:
a. at $30 \mathrm{~cm}$ behind configuration
b. at $304.8 \mathrm{~cm}$ from reactor centerline (Foreground and Background).

3. Bare detector on centerline at $30 \mathrm{~cm}$ behind configuration inside partial cadmium enclosure that surrounded the detector except on the side of the last slab in the configuration.

4. Bare detector on centerline at $304.8 \mathrm{~cm}$ point with cadmium sheet over face of last slab in the configuration.

C. $\mathrm{SM} 1+1.27 \mathrm{~cm} \mathrm{Al}+15.24 \mathrm{~cm} \mathrm{SS}+30.48 \mathrm{~cm}$ graphite

1. Bare, cd-covered, 3-, 5-, 8-, 10-in BB on centerline:
a. at $30 \mathrm{~cm}$ behind configuration
b. at $304.8 \mathrm{~cm}$ from reactor centerline (Foreground and Background).

2. Bare detector on centerline at $30 \mathrm{~cm}$ behind configuration inside partial cadmium enclosure that surrounded the detector except on the side of the last slab in the configuration.

3. Bare detector on centerline at $304.8 \mathrm{~cm}$ point with cadmium sheet over face of last slab in the configuration.

D. $\quad \mathrm{SM1}+1.27 \mathrm{~cm} \mathrm{Al}+15.24 \mathrm{~cm} \mathrm{SS}+40.64 \mathrm{~cm}$ graphite

1. 5-in $\mathrm{BB}$ horizontal traverse at $30 \mathrm{~cm}$ behind configuration.

2. Bare, cd-covered, 3-, 5-, 8-, 10-in BB on centerline:
a. at $30 \mathrm{~cm}$ behind configuration
b. at $304.8 \mathrm{~cm}$ from reactor centerline (Foreground and Background).


3. Bare detector on centerline at $30 \mathrm{~cm}$ behind configuration inside partial cadmium enclosure that surrounded the detector except on the side of the last slab in the configuration.

4. Bare detector on centerline at $304.8 \mathrm{~cm}$ point with cadmium sheet over face of last slab in the configuration.

5. INS ${ }^{3}$ He $\mathrm{BB}$ measurements on centerline:

a. at $30 \mathrm{~cm}$ behind configuration

b. at $304.8 \mathrm{~cm}$ from reactor centerline (Foreground and Background) .

E. $\quad \mathrm{SM} 1+1.27 \mathrm{~cm} \mathrm{Al}+15.24 \mathrm{~cm} \mathrm{SS}+50.8 \mathrm{~cm}$ graphite

1. Bare, cd-covered, 3-, 5-, 8-, 10-in BB on centerline:

a. at $30 \mathrm{~cm}$ behind configuration

b. at $304.8 \mathrm{~cm}$ from reactor centerline (Foreground and Background).

2. Bare detector on centerline at $30 \mathrm{~cm}$ behind configuration inside partial cadmium enclosure that surrounded the detector except on the side of the last slab in the configuration.

3. Bare detector on centerline at $304.8 \mathrm{~cm}$ point with cadmium sheet over face of last slab in the configuration.

F. $\quad \mathrm{SM} 1+1.27 \mathrm{~cm} \mathrm{Al}+15.24 \mathrm{~cm} \mathrm{SS}+60.96 \mathrm{~cm}$ graphite

1. NE-213 and hydrogen counter measurements on centerline as close as feasible behind SS.

2. 3-, 5-, 10-in BB measurements on centerline at same location as $\mathrm{NE}-213$.

3. 5-in $B B$ horizontal traverse at $30 \mathrm{~cm}$ behind configuration.

4. Bare, cd-covered, 3-, 5-, 8-, 10-in BB on centerline:

a. at $30 \mathrm{~cm}$ behind configuration

b. at $304.8 \mathrm{~cm}$ from reactor centerline (Foreground and Background).

5. Bare detector on centerline at $30 \mathrm{~cm}$ behind configuration inside partial cadmium enclosure that surrounded the detector except on the side of the last slab in the configuration. 
6. Bare detector on centerline at $304.8 \mathrm{~cm}$ point with cadmium sheet over face of last slab in the configuration.

7. INS ${ }^{3} \mathrm{He}$ BB measurements on centerline:

a. at $30 \mathrm{~cm}$ behind configuration

b. at $304.8 \mathrm{~cm}$ from reactor centerline (Foreground and Background).

VII. SM + SS and $\mathrm{B}_{4} \mathrm{C}$ (near core shield)

A. SM1 $(10.27 \mathrm{~cm} \mathrm{Fe}+9.17 \mathrm{~cm} \mathrm{Al}+2.54 \mathrm{~cm}$ boral $+20.32 \mathrm{~cm}$ radial blanket) $+1.27 \mathrm{~cm} \mathrm{Al}+15.24 \mathrm{~cm} \mathrm{SS}+1.27 \mathrm{~cm} \mathrm{Al}+15.24 \mathrm{~cm} \mathrm{SS}$

1. 5-in $B B$ horizontal traverse at $30 \mathrm{~cm}$ behind configuration.

2. 3-, 5-, 8-, 10-in BB measurements on centerline:

a. at $30 \mathrm{~cm}$ behind configuration

b. at $304.8 \mathrm{~cm}$ from reactor centerline (Foreground and Background).

B. $\mathrm{SM} 1+1.27 \mathrm{~cm} \mathrm{Al}+15.24 \mathrm{~cm} \mathrm{SS}+1.27 \mathrm{~cm} \mathrm{Al}+15.24 \mathrm{~cm} \mathrm{SS}+1.27 \mathrm{~cm} \mathrm{Al}$ $+15.24 \mathrm{~cm}$ SS

1. 5 -in $\mathrm{BB}$ horizontal traverse at $30 \mathrm{~cm}$ behind configuration.

2. 3-, 5-, 8-, 10-in BB measurements on centerline:

a. at $30 \mathrm{~cm}$ behind configuration

b. at $304.8 \mathrm{~cm}$ from reactor centerline (Foreground and Background).

C. $\quad \mathrm{SM} 1+1.27 \mathrm{~cm} \mathrm{Al}+15.24 \mathrm{~cm} \mathrm{SS} \div 1.27 \mathrm{~cm} \mathrm{Al}+15.24 \mathrm{~cm} \mathrm{SS}+1.27 \mathrm{~cm} \mathrm{Al}$

$+15.24 \mathrm{~cm} \mathrm{SS}+1.27 \mathrm{~cm} \mathrm{Al}+15.24 \mathrm{~cm} \mathrm{~B} \mathrm{~B}_{4} \mathrm{C}+2.54 \mathrm{~cm} \mathrm{SS}$

1. 5-in $B B$ horizontal traverse at $30 \mathrm{~cm}$ behind configuration.

2. 3-, 5-, 8-, 10-in BB measurements on centerline:

a. at $30 \mathrm{~cm}$ behind configuration

b. at $304.8 \mathrm{~cm}$ from reactor centerline (Foreground and Background).

3. INS ${ }^{3} \mathrm{He} \mathrm{BB}$ measurements on centerline:

a. at $30 \mathrm{~cm}$ behind configuration 
b. at $304.8 \mathrm{~cm}$ from reactor centerline (Foreground and Background).

D. $\quad \mathrm{SM} 1+1.27 \mathrm{~cm} \mathrm{Al}+15.24 \mathrm{~cm} \mathrm{SS}+1.27 \mathrm{~cm} \mathrm{Al}+15.24 \mathrm{~cm} \mathrm{SS}+1.27 \mathrm{~cm} \mathrm{Al}$

$+15.24 \mathrm{~cm} \mathrm{SS}+1.27 \mathrm{~cm} \mathrm{Al}+15.24 \mathrm{~cm} \mathrm{~B} 4 \mathrm{C}+1.54 \mathrm{~cm} \mathrm{SS}+30.48 \mathrm{~cm} \mathrm{Na}$

1. Bare, cd-covered, 3-, 5-, 8-, 10-in BB measurements on centerline:

a. at $30 \mathrm{~cm}$ behind configuration

b. at $304.8 \mathrm{~cm}$ from reactor centerline (Foreground and Background).

2. Bare detector on centerline at $30 \mathrm{~cm}$ behind configuration inside partial cadmium enclosure that surrounded the detector except on the side of the last slab in the configuration.

3. Bare detector on centerline at $304.8 \mathrm{~cm}$ point with cadmium shell over face of last slab in the configuration. 


\section{APPENDIX B. TABLES OF DATA}

\section{List of Tables}

Table 1. Analysis of iron slabs used in spectrum modifier

Table 2. Analysis of aluminum slabs used in spectrum modifier

Table 3. Composition of boral slabs used in spectrum modifier

Table 4. Composition of $\mathrm{vO}_{2}$ radial blanket

Table 5. Analysis of aluminum used in $\mathrm{VO}_{2}$ radial blanket cladding

Table 6. Composition of lithiated-paraffin bricks

Table 7. Analysis of $61-\mathrm{cm} \times 61-\mathrm{cm} \times 30.5-\mathrm{cm}$ concrete blocks used to surround configuration

Table 8. Composition of sodium slabs

Table 9. Analysis of type 304 stainless steel slabs used in radial shield

Table 10. Analysis of boron carbide used in shield mockups

Table 11. Analysis of graphite used in shield mockup

Table 12. Fast neutron fluxes $(>0.8 \mathrm{MeV})$ on centerline at $178.8 \mathrm{~cm}$ behind the configuration (Item IA): Run 7855A

Table 13. Neutron fluxes (50 keV to $1.4 \mathrm{MeV}$ ) on centerline $178.8 \mathrm{~cm}$ beyond the SM (Item IA): Runs 1537C, 1537A, 1536A

Table 14. Bonner ball measurements on centerline at NE-213 location (Items IA, IID, IVC, VIA, F)

Table 15. Bonner ball measurements on centerline at $30 \mathrm{~cm}$ behind a series of configurations (Items IA, IIA-E, IIIA-E, IVA-I)

Table 16. Bonner ball measurements on centerline at $304.8 \mathrm{~cm}$ or $457.2 \mathrm{~cm}$ from the center of the reactor (Items IA, IIA-E, IIIA-E, IVA-F)

Table 17. 5-inch Bonner ball horizontal traverses through midplane at 30 $\mathrm{cm}$ behind a series of configurations (Items IA, IIA, D, E, IIIA, B, C, E)

Table 18. Fast neutron fluxes $(>0.8 \mathrm{MeV})$ on centerline at $47.3 \mathrm{~cm}$ behind the $\mathrm{B}_{4} \mathrm{C}(33.0 \mathrm{~cm}$ behind lead) (Item IID): Run $7857 \mathrm{~A}$

Table 19. Neutron fiuxes (50 keV to $1.4 \mathrm{MeV})$ on centerline $47.3 \mathrm{~cm}$ beyond $\mathrm{B}_{4} \mathrm{C}(33.0 \mathrm{~cm}$ beyond $\mathrm{Pb})$ (Item IID): Runs 1540A, 1539B, 1539A

Table 20. 5-inch Bonner ball horizontal traverse through midplane at $30 \mathrm{~cm}$ behind a series of configurations (Items IVA, C, G)

Table 21. Fast neutron fluxes $(>0.8 \mathrm{MeV})$ on centerline $42.8 \mathrm{~cm}$ beyond ss $(32.9 \mathrm{~cm}$ beyond $\mathrm{Pb})$ (Item IVC): Run $7860 \mathrm{~A}$

Table 22. Neutron fluxes (50 keV to $1.4 \mathrm{MeV}$ ) on centerline $42.8 \mathrm{~cm}$ beyond SS $(32.9 \mathrm{~cm}$ beyond $\mathrm{Pb}$ ) (Item IVC): Runs 1546A, 1546B, $1547 \mathrm{~A}$ 
Table 23. Bonner ball measurements on centerline behind a series of configurations (Items IVF-I)

Table 24. Bonner ball measurements on centerline midway in the void between Na tanks (Items IVI-M)

Table 25. 5-inch Bonner ball traverses through horizontal midplane $30 \mathrm{~cm}$ behind a series of configurations (Items VA, B, D, G, J)

Table 26. Bonner ball measurements on centerline at $30 \mathrm{~cm}$ behind a series of configurations (Items VA-J)

Table 27. Bonner ball measurements on centerline at 304.8 or $501.3 \mathrm{~cm}$ from the center of the reactor (Items VA-J, VIA-F, VIIA-D)

Table 28. Fast neutron fluxes $(>0.8 \mathrm{MeV})$ on centerline at $51.1 \mathrm{~cm}$ behind the graphite $(36.4 \mathrm{~cm}$ behind the $\mathrm{Pb}$ ) (Item VIA): Run $7856 \mathrm{~A}$

Table 29. Neutron fluxes (50 keV to $1.4 \mathrm{MeV})$ on centerline at $51.1 \mathrm{~cm}$ behind the graphite $(36.4 \mathrm{~cm}$ behind the $\mathrm{Pb})$ (Item VIA): Runs $1542 \mathrm{~A}, 1541 \mathrm{~B}, 1541 \mathrm{~A}$

Table 30. 5-inch Bonner ball horizontal traverses through midplane at 30 $\mathrm{cm}$ behind a series of configurations (Items VIA, B, D, F, VIIAc)

Table 31. Bonner ball measurements on centerline at $30 \mathrm{~cm}$ behind a series of configurations (Items VIA-F, VIIA-D)

Table 32. Fast neutron fluxes $(>0.8 \mathrm{MeV})$ on centerline at $63.2 \mathrm{~cm}$ beyond the graphite in the configuration (Item VIF): Run 7859A

Table 33. Neutron fluxes $(50 \mathrm{keV}$ to $1.4 \mathrm{MeV})$ on centerline at $63.2 \mathrm{~cm}$ beyond the graphite in the configuration (Item VIF): Runs $1544 \mathrm{~A}, 1544 \mathrm{~B}, 1545 \mathrm{~A}$ 
Table 1. Analysis of iron slabs $(\rho 7.86 \mathrm{~g} / \mathrm{cc})$ used in spectrum modifier

\begin{tabular}{lc}
\hline Element & wt $\%$ \\
\hline $\mathrm{Fe}$ & 98.4 \\
$\mathrm{C}$ & .25 \\
$\mathrm{Cr}$ & .15 \\
$\mathrm{Cu}$ & .03 \\
$\mathrm{Mn}$ & 1.0 \\
$\mathrm{Mo}$ & .02 \\
$\mathrm{Ni}$ & .05 \\
$\mathrm{Si}$ & .25 \\
\hline
\end{tabular}

Table 2. Analysis of aluminum slabs ( $2.70 \mathrm{~g} / \mathrm{cc}$ ) used in spectrum modifier

\begin{tabular}{ccr}
\hline Element & wt \% & ppm \\
\hline $\mathrm{Al}$ & 97.5 & \\
$\mathrm{Cr}$ & .22 & \\
$\mathrm{Cu}$ & .23 & \\
$\mathrm{Fe}$ & .47 & \\
$\mathrm{Mg}$ & .86 & \\
$\mathrm{Mn}$ & .01 & \\
$\mathrm{Si}$ & .63 & \\
$\mathrm{Ti}$ & .042 & \\
$\mathrm{Zn}$ & .07 & \\
$\mathrm{Li}$ & & 3 \\
$\mathrm{Ni}$ & & 50 \\
$\mathrm{Sn}$ & & $<10$ \\
$\mathrm{~V}$ & & 150 \\
& & \\
\hline
\end{tabular}


Table 3. Composition of boral slabs used in spectrum modifier

\begin{tabular}{|c|c|c|c|c|}
\hline \multirow[b]{2}{*}{ Component } & \multicolumn{4}{|c|}{$\left(\mathrm{B}_{4} \mathrm{C}-40-43\right.$ vol $\%$ in $\mathrm{B}_{4} \mathrm{C}-\mathrm{Al}$ mixture) } \\
\hline & $\begin{array}{r}\text { Density } \\
(\mathrm{g} / \mathrm{cc})\end{array}$ & $\begin{array}{c}\text { Elemental } \\
\text { Composition } \\
\text { (wt \%) }\end{array}$ & $\mathrm{Al}$ & $\begin{array}{l}\text { With } \\
\text { Cladding } \\
(\text { wt \%) }\end{array}$ \\
\hline $\mathrm{B}_{4} \mathrm{C}$ & 2.3 & & & \\
\hline Al & 2.70 & 65 & & $\sim 75$ \\
\hline$B$ & & 27.5 & & $\sim 19.6$ \\
\hline c & & 7.5 & & $\approx 5.4$ \\
\hline
\end{tabular}


Table 4. Composition of $\mathrm{UO}_{2}$ radial blanket

\begin{tabular}{|c|c|c|c|c|c|}
\hline \multicolumn{2}{|c|}{ Component } & vol & $\%$ & & $\begin{array}{r}\text { Densi } \\
(\mathrm{g} / \mathrm{c}\end{array}$ \\
\hline \multicolumn{2}{|c|}{$\begin{array}{l}\mathrm{UO}_{2} \text { (pellets) } \\
\mathrm{Al}^{2}(8001) \\
\mathrm{Na} \\
\text { Void }\end{array}$} & $\begin{array}{r}64.6 \\
11.2 \\
23.2 \\
1.0\end{array}$ & & & $\begin{array}{r}10.2 \\
2.8 \\
0.9 \\
-\end{array}$ \\
\hline \multicolumn{6}{|c|}{$\mathrm{UO}_{2}$ content $88.18 \mathrm{wt} \%$} \\
\hline \multicolumn{6}{|c|}{ Isotope \% } \\
\hline \multicolumn{2}{|c|}{$\begin{array}{l}234 \mathrm{U} \\
235 \mathrm{U}\end{array}$} & & $\begin{array}{l}236_{U} \\
238_{U}\end{array}$ & \multicolumn{2}{|r|}{99.28} \\
\hline \multicolumn{2}{|c|}{ Metallic } & rit & ies in & $\mathrm{O}_{2}$ & (ppm) \\
\hline Al & $<20$ & $\mathrm{Cu}$ & 1 & $\mathrm{Na}$ & $<20$ \\
\hline$B$ & $<1$ & $\mathbf{F}$ & $<2$ & $\mathrm{Ni}$ & $<10$ \\
\hline $\mathrm{Be}$ & $<2$ & $\mathrm{Fe}$ & $<20$ & $\mathrm{~Pb}$ & $<4$ \\
\hline $\mathrm{Bi}$ & $<2$ & $\mathrm{H}_{2} \mathrm{O}$ & 2.1 & Si & $<20$ \\
\hline$C$ & $<10$ & L & $<1$ & $\mathrm{Sn}$ & $<2$ \\
\hline $\mathrm{Ca}$ & $<20$ & $\mathrm{Mg}$ & $<10$ & $\mathrm{Ta}$ & $<25$ \\
\hline $\mathrm{Cd}$ & $<.5$ & $\mathrm{Mn}$ & $<4$ & $\mathrm{Tu}$ & $<4$ \\
\hline $\mathrm{Cl}$ & $<3.3$ & Mo & $<10$ & $w$ & $<25$ \\
\hline Co & $<2$ & $\mathrm{~N}$ & 54 & $\mathrm{Zr}$ & $<25$ \\
\hline $\mathrm{Cr}$ & $<10$ & & & & \\
\hline
\end{tabular}


Table 5. Analysis of aluminum used in $\mathrm{vO}_{2}$ radial blanket cladding

\begin{tabular}{lcc}
\hline Element & wt \% & ppm \\
& & \\
$\mathrm{Fe}$ & .59 & \\
$\mathrm{Ni}$ & 1.13 & \\
$\mathrm{~B}$ & & $<6$ \\
$\mathrm{Be}$ & $<20$ \\
$\mathrm{Cd}$ & $<20$ \\
$\mathrm{Co}$ & $<20$ \\
$\mathrm{Cr}$ & $<6$ \\
$\mathrm{Cu}$ & 52.9 \\
$\mathrm{Li}$ & 6 \\
$\mathrm{Ng}$ & 3.04 \\
$\mathrm{Mn}$ & 11.2 \\
$\mathrm{Mo}$ & $<6$ \\
$\mathrm{~Pb}$ & $<20$ \\
$\mathrm{Si}$ & 27.5 \\
$\mathrm{Sn}$ & $<60$ \\
$\mathrm{~T}$ & $<2000$ \\
$\mathrm{Ti}$ & 65.5 \\
$\mathrm{~V}$ & 44.2 \\
$\mathrm{~W}$ & $<60$ \\
$\mathrm{Zr}$ & $<20$ \\
& \\
\hline
\end{tabular}

Table 6. Composition of lithiated-paraffin bricks $(\rho 1.15 \mathrm{~g} / \mathrm{cc})$

\begin{tabular}{lc}
\hline Component & wt\% \\
\hline $\mathrm{CH}_{4}$ & 60 \\
$\mathrm{LiCO}_{3}$ & 40 \\
\hline
\end{tabular}


Table 7. Analysis of $61-\mathrm{cm} \times 61-\mathrm{cm} \times 30.5-\mathrm{cm}(\rho 2.4 \mathrm{~g} / \mathrm{cc})$ concrete blocks used to surround configuration

\begin{tabular}{lcll}
\hline Component & wt $\%$ & Component & wt \% \\
\hline $\mathrm{Co}_{3}$ & 41.9 & $\mathrm{Al}_{2} \mathrm{O}_{3}$ & 2.2 \\
$\mathrm{Ca}$ & 27.4 & $\mathrm{Fe}_{2} \mathrm{O}_{3}$ & .60 \\
$\mathrm{SiO}_{2}$ & 18.1 & $\mathrm{SO}_{3}$ & .32 \\
$\mathrm{H}_{2} \mathrm{O}^{2}$ & 4.0 & $\mathrm{P}_{2} \mathrm{O}_{5}$ & .035 \\
$\mathrm{Mg}$ & 3.66 & $\mathrm{~K}$ & .30 \\
$\mathrm{O}_{2}$ & 1.4 & & \\
\hline
\end{tabular}

Table 8. Composition of sodium slabs $(\rho 0.945 \mathrm{~g} / \mathrm{cc})$

\begin{tabular}{lr}
\hline Element & wt \% \\
\hline $\mathrm{Na}$ & 99.7 \\
$\mathrm{Ca}, \mathrm{Zn}$ & 0.3 \\
\hline
\end{tabular}


Table 9. Analysis of type 304 stainless steel slabs used in radial shield

\begin{tabular}{lcc}
\hline Element & $\begin{array}{c}2.54-\mathrm{cm}-\text { thick } \\
(\text { wt \%) }\end{array}$ & $\begin{array}{c}5.08-\mathrm{cm}-\text { thick } \\
(\text { wt \%) }\end{array}$ \\
\hline $\mathrm{Fe}$ & 71.2 & $68.1-69.3$ \\
$\mathrm{Cr}$ & 18.3 & $18.7-19.1$ \\
$\mathrm{Ni}$ & 8.8 & $9.6-9.8$ \\
$\mathrm{Mn}$ & 1.3 & $1.04-1.65$ \\
$\mathrm{Si}$ & .35 & $.33-.65$ \\
$\mathrm{C}$ & .039 & $.024-.085$ \\
$\mathrm{O}_{2}$ & .015 & $.013-.021$ \\
$\mathrm{P}$ & .028 & .028 \\
$\mathrm{~S}$ & .022 & .022 \\
$\mathrm{Mo}$ & .30 & .30 \\
$\mathrm{Cu}$ & .26 & .26 \\
$\mathrm{Co}$ & .10 & .10 \\
\hline
\end{tabular}

Table 10. Analysis of boron carbide used in shield mockups

\begin{tabular}{llr}
\hline Element & wt \% & ppm \\
\hline & & \\
B & 76.7 & \\
$\mathrm{C}$ & 19.52 & \\
$\mathrm{Al}$ & & 50 \\
$\mathrm{Ca}$ & 800 \\
$\mathrm{Cl}$ & & 10 \\
$\mathrm{Co}$ & & $<1$ \\
$\mathrm{Cr}$ & 2 \\
$\mathrm{Cu}$ & & $<1$ \\
$\mathrm{Fe}$ & & 600 \\
$\mathrm{Mg}$ & & 25 \\
$\mathrm{Mn}$ & 10 \\
$\mathrm{Na}$ & & 1 \\
$\mathrm{~Pa}$ & 2 \\
$\mathrm{~S}$ & & 5 \\
$\mathrm{Si}$ & & 50 \\
$\mathrm{Ti}$ & & 25 \\
\hline
\end{tabular}


Table 11. Analysis of graphite used in shield mockup $(\rho 1.62 \mathrm{~g} / \mathrm{cc})$

\begin{tabular}{|c|c|c|c|c|c|}
\hline $\mathrm{Ag}$ & $<\quad 0.5$ & $\mathrm{Ge}$ & $<5$ & $\mathrm{Sb}$ & $<10$ \\
\hline $\mathrm{Al}$ & 12.5 & $\mathrm{Hg}$ & $<10$ & $\mathrm{Si}$ & 100 \\
\hline B & 1.5 & In & $<10$ & $\mathrm{Sn}$ & $<10$ \\
\hline $\mathrm{Ba}$ & $<5$ & $\mathbf{K}$ & $<100$ & $\mathrm{Sr}$ & $<10$ \\
\hline $\mathrm{Be}$ & $<0.5$ & $\mathrm{Li}$ & $<2$ & $\mathrm{Ta}$ & $<10$ \\
\hline $\mathrm{Bi}$ & $<10$ & $\mathrm{Mg}$ & 7.5 & $\mathrm{Te}$ & $<25$ \\
\hline $\mathrm{Ca}$ & 150 & Mn & $<\quad 1$ & $\mathrm{Ti}$ & 30 \\
\hline $\mathrm{cd}$ & $<25$ & Mo & $<5$ & V & 100 \\
\hline Co & $<10$ & $\mathrm{Na}$ & $<5$ & $\mathbf{W}$ & $<5$ \\
\hline $\mathrm{Cr}$ & $<10$ & $\mathrm{Nb}$ & $<5$ & $\mathrm{Zn}$ & 10 \\
\hline $\mathrm{Cu}$ & 7.5 & $\mathrm{Ni}$ & $<10$ & $\mathrm{Zr}$ & $<5$ \\
\hline $\mathrm{Fe}$ & 50 & $\mathrm{~Pb}$ & $<5$ & & \\
\hline $\mathrm{Ga}$ & $<10$ & $\mathrm{Rb}$ & $<5$ & & \\
\hline
\end{tabular}


Table 12. Fast Neutron fluxes $(>0.8 \mathrm{MeV})$ on centerline at $178.8 \mathrm{~cm}$ behind the configuration (Item IA): Run 7855A

\begin{tabular}{|c|c|c|c|c|c|c|c|c|c|}
\hline \multicolumn{2}{|c|}{$\begin{array}{l}\text { Neutron } \\
\text { Energy } \\
(\text { MeV })\end{array}$} & \multicolumn{2}{|c|}{$\begin{array}{l}\text { Flux (neutrons } \\
\text { Lower } \\
\text { Limit }\end{array}$} & $\begin{array}{c}\mathrm{cm}^{-2} \mathrm{MeV}^{-1} \mathrm{~kW}^{-1} \mathrm{~s}^{-1} \text { ) } \\
\text { Upper } \\
\text { Limit }\end{array}$ & $\begin{array}{l}\text { Neutron } \\
\text { Energy } \\
\text { (MeV) }\end{array}$ & \multicolumn{2}{|c|}{$\begin{array}{l}\text { Flux (neutrons } \\
\text { lower } \\
\text { Limit }\end{array}$} & \multicolumn{2}{|c|}{$\begin{array}{c}\mathrm{cm}^{-2} \mathrm{MeV}^{-1} \mathrm{~kW}^{-1} \mathrm{~s}^{-1} \\
\text { Upper } \\
\text { Limit }\end{array}$} \\
\hline $8.11 \mathrm{E}$ & $(-1)$ & & $1.79 \mathrm{E} \quad(4)$ & $1.81 \mathrm{~F}(4)$ & $5.94 \mathrm{E} \quad(0)$ & $4.61 \mathrm{E}$ & (2) & $4.76 \mathrm{E}$ & (2) \\
\hline $9.07 \mathrm{E}$ & $(-1)$ & & $1.68 \mathrm{E}(4)$ & $1.70 \mathrm{E}$ (4) & $6.25 \mathrm{E}(0)$ & $3.98 \mathrm{E}$ & (2) & $4.19 E$ & (2) \\
\hline $1.01 \mathrm{E}$ & $(0)$ & & $1.36 \mathrm{E}$ (4) & $1.37 \mathrm{E}$ (4) & $6.56 \mathrm{~F} \quad(0)$ & $3.33 \mathrm{E}$ & (2) & $3.51 \mathrm{E}$ & (2) \\
\hline $1.11 \mathrm{E}$ & $(0)$ & & $1.18 \mathrm{~F},(4)$ & $1.19 \mathrm{E}$ (4) & $6.84 \mathrm{E}(0)$ & $2.63 \mathrm{E}$ & (2) & $2.75 E$ & (2) \\
\hline $1.20 E$ & $(0)$ & & $1.11 \mathrm{E}$ (4) & $1.12 \mathrm{E}$ (4) & $7.24 \mathrm{E}(0)$ & $1.80 \mathrm{E}$ & (2) & $1.89 E$ & (2) \\
\hline $1.31 E$ & $(0)$ & & $1: 06 \mathrm{E}$ (4) & $1.06 \mathrm{E}(4)$ & $7.74 E \quad(0)$ & $1.63 \mathrm{E}$ & (2) & $1.71 \mathrm{E}$ & (2) \\
\hline $1.41 \mathrm{E}$ & $(0)$ & & $9.85 E \quad(3)$ & $9.94 \mathrm{E}$ (3) & $8.24 \mathrm{E} \quad(0)$ & $1.72 \mathrm{E}$ & (2) & $1.76 \mathrm{E}$ & (2) \\
\hline $1.51 \mathrm{E}$ & (0) & & $9.08 \mathrm{E}$ (3) & $9.16 \mathrm{E} \quad(3)$ & $8.76 \mathrm{E} \quad(0)$ & $1.18 \mathrm{E}$ & (2) & $1.22 \mathrm{E}$ & (2) \\
\hline $1.61 \mathrm{E}$ & $(0)$ & & $8.27 \mathrm{E}$ (3) & $8.35 F \quad$ (3) & $9.26 \mathrm{E}(0)$ & $5.52 E$ & (1) & $5.73 \mathrm{E}$ & (1) \\
\hline $1.71 \mathrm{E}$ & (0) & & $7.54 \mathrm{E} \quad(3)$ & $7.61 \mathrm{E}$ (3) & $9.74 \mathrm{E} \quad(0)$ & $2.06 \mathrm{E}$ & (1) & $2.15 \mathrm{E}$ & (1) \\
\hline $1.81 \mathrm{E}$ & (0) & & $6.96 \mathrm{E} \quad(3)$ & $7.04 \mathrm{E} \quad(3)$ & $1.03 \mathrm{E}$ (1) & $6.36 \mathrm{E}$ & (0) & $6.67 \mathrm{E}$ & $(0)$ \\
\hline $1.93 \mathrm{E}$ & (0) & & $6.52 \mathrm{E} \quad(3)$ & $6.60 \mathrm{E} \quad(3)$ & $1.08 \mathrm{E} \quad(1)$ & $2.20 \mathrm{E}$ & (0) & $2.39 E$ & $(0)$ \\
\hline $2.10 \mathrm{E}$ & $(0)$ & & $6.08 \mathrm{~F} \quad(3)$ & $6.14 \mathrm{~F}$ (3) & $1.12 \mathrm{~F}$ (1) & $1.05 \mathrm{E}$ & $(0)$ & $1.20 \mathrm{E}$ & $(0)$ \\
\hline $2.30 E$ & $(0)$ & & $5.44 E \quad(3)$ & $5.50 \mathrm{E}$ (3) & $1,18 E$ & $5.57 \mathrm{E}$ & $(-1)$ & $6.45 E$ & $(-1)$ \\
\hline $2.50 \mathrm{E}$ & $(0)$ & & $4.64 \mathrm{~F} \quad(3)$ & $4.69 \mathrm{E}$ (3) & $1.24 \mathrm{E}(1)$ & $2.49 \mathrm{E}$ & $(-1)$ & $3.07 \mathrm{E}$ & $(-1)$ \\
\hline $2.70 \mathrm{E}$ & $(0)$ & & $3.77 \mathrm{E}$ (3) & $3.82 \mathrm{E}$ (3) & $1.32 \mathrm{E}(1)$ & $7.12 \mathrm{E}$ & $(-2)$ & $1.08 \mathrm{E}$ & $(-1)$ \\
\hline $2.90 \mathrm{E}$ & $(0)$ & & $2.96 \mathrm{E} \quad(3)$ & $3.01 \mathrm{E}$ (3) & $1.40 \mathrm{~F}$ (1) & $1.05 \mathrm{E}$ & $(-2)$ & $2.47 E$ & $(-2)$ \\
\hline $3.10 E$ & $(0)$ & & $2.26 \mathrm{E}$ (3) & $2.31 \mathrm{E}$ (3) & $1.48 \mathrm{E}(1)$ & $-2.39 E$ & $(-3)$ & $1.84 \mathrm{E}$ & $(-3)$ \\
\hline $3.30 \mathrm{E}$ & $(0)$ & & $1.77 \mathrm{E} \quad(3)$ & $1.81 \mathrm{E} \quad(3)$ & $1.56 \mathrm{~F}(1)$ & $-3.49 \mathrm{E}$ & $(-3)$ & $-2.48 E$ & $(-3)$ \\
\hline $3.50 \mathrm{E}$ & $(0)$ & & $1.46 E \quad(3)$ & $1.51 \mathrm{E}$ (3) & $1.65 \mathrm{E} \quad(1)$ & $-2.74 \mathrm{E}$ & $(-3)$ & $-2.33 \mathrm{E}$ & $(-3)$ \\
\hline $3.71 \mathrm{E}$ & (0) & & $1.29 \mathrm{E}$ (3) & $1.33 E \quad(3)$ & $1.75 \mathrm{~F}$ (1) & $-2.19 \mathrm{E}$ & $(-3)$ & $-1.76 \mathrm{E}$ & $(-3)$ \\
\hline $3.91 \mathrm{E}$ & $(0)$ & & $1.18 \mathrm{E}(3)$ & $1.22 E \quad(3)$ & $1.85 \mathrm{E}(1)$ & $-1.71 E$ & $(-3)$ & $-1.22 \mathrm{E}$ & $(-3)$ \\
\hline $4.15 \mathrm{E}$ & $(0)$ & & $1.09 \mathrm{E}$ (3) & $1.12 \mathrm{E} \quad(3)$ & $1.95 \mathrm{~F} \quad(1)$ & $-1.23 \mathrm{E}$ & $(-3)$ & $-7.49 \mathrm{E}$ & $(-4)$ \\
\hline $4.45 E$ & (0) & & $9.88 \mathrm{E}(2)$ & $1.01 E \quad(3)$ & $2.06 \mathrm{E}$ (1) & $-8.04 \mathrm{E}$ & $(-4)$ & $-3.33 E$ & $(-4)$ \\
\hline $4.75 \mathrm{E}$ & (0) & & $8.73 E$ (2) & $8.96 \mathrm{E} \quad$ (2) & $2.16 \mathrm{E}$ & $-4.60 \mathrm{E}$ & $(-4)$ & $-9.03 E$ & $(-6)$ \\
\hline $5.04 E$ & $(0)$ & & $7.49 \mathrm{E}$ (2) & $7.69 \mathrm{E}$ (2) & $2.26 E$ (1) & $-3.27 E$ & $(-4)$ & $1.28 \mathrm{E}$ & $(-4)$ \\
\hline $5.35 \mathrm{E}$ & $(0)$ & & $6.22 \mathrm{~F},(2)$ & $6.41 \mathrm{E} \quad(2)$ & $2.35 \mathrm{E} \quad(1)$ & $-2.64 \mathrm{E}$ & $(-4)$ & $2.22 F$ & $(-4)$ \\
\hline $5.65 E$ & $(0)$ & & $5.24 E$ (2) & $5.43 \mathrm{E}$ (2) & & & & & \\
\hline
\end{tabular}

\begin{tabular}{|c|c|c|c|}
\hline $\begin{array}{c}\mathrm{E} 1 \\
(\mathrm{MeV})\end{array}$ & $\begin{array}{l}\mathrm{E} 2 \\
(\mathrm{MeV})\end{array}$ & $\begin{array}{c}\text { Integral } \\
\text { (neutrons } \mathrm{cm}^{-2} \mathrm{~kW}^{-1} \mathrm{~s}^{-1} \text { ) }\end{array}$ & $\begin{array}{l}\text { Error } \\
\text { (neutrons } \mathrm{cm}^{-2} \mathrm{~kW}^{-1} \mathrm{~s}^{-1} \text { ) }\end{array}$ \\
\hline 0.811 & 1.000 & $3.13 \mathrm{E}$ & $1.36 \mathrm{~F}$ \\
\hline 1.000 & 1.200 & $2.44 \mathrm{E}$ & 1.03E (1) \\
\hline 1.200 & 1.600 & $3.97 \mathrm{~F}$ & $1.74 \mathrm{~F}$ \\
\hline 1.600 & 2.000 & $2.87 \mathrm{E}$ & $1.46 \mathrm{E}$ \\
\hline 2.000 & 3.000 & $4.60 \mathrm{E}$ & $2.76 \mathrm{E}$ \\
\hline 3.000 & 4.000 & $1.62 \mathrm{E}$ & $1.99 \mathrm{E}$ \\
\hline 4.000 & 6.000 & $1.57 \mathrm{E}$ & $2.16 \mathrm{E}$ \\
\hline 6.000 & 8.000 & $5.27 E$ & $1.25 \mathrm{E}$ \\
\hline 8.000 & 10.000 & $1.85 \mathrm{E}$ & $2.91 \%$ \\
\hline 10.000 & 12.000 & $5.83 \mathrm{E}$ & $1.93 E(-1)$ \\
\hline 12.000 & 16.000 & $3.22 \mathrm{~F} \quad(-1)$ & $4.64 \mathrm{E} \quad(-2)$ \\
\hline 16.000 & 20.000 & $7.02 \mathrm{E} \quad(-3)$ & $9.45 \mathrm{E} \quad(-4)$ \\
\hline 1.500 & 15.000 & 1.23E & $1.03 \mathrm{E}$ \\
\hline 3.000 & 12.000 & $3.91 \mathrm{E}$ & $5.70 E$ \\
\hline
\end{tabular}


Table 13. Neutron fluxes (50 keV to $1.4 \mathrm{MeV}$ ) on centerline $178.8 \mathrm{~cm}$ beyond the SM (Item IA): Runs 1537C, 1537A, 1536A

\begin{tabular}{|c|c|c|c|c|}
\hline $\mathrm{N}$ & \multicolumn{2}{|c|}{$\begin{array}{c}\text { Energy Boundary } \\
(\mathrm{MeV})\end{array}$} & \multirow[t]{2}{*}{ (Neutron $\mathrm{cm}^{-\frac{\text { Flux }}{2}} \mathrm{MeV}^{-1} \mathrm{~kW}^{-1} \mathrm{~s}^{-1}$ ) } & \multirow[t]{2}{*}{$\begin{array}{c}\text { Error } \\
(\%)\end{array}$} \\
\hline & & & & \\
\hline 1 & 0.0548 & 0.0635 & $7.57 \mathrm{E} \quad 05$ & 1.94 \\
\hline 2 & 0.0635 & 0.0756 & $6.83 \mathrm{E} \quad 05$ & 1.62 \\
\hline 3 & 0.0756 & 0.0896 & $5.70 E \quad 05$ & 1.83 \\
\hline 4 & 0.0896 & 0.1052 & $3.93 \mathrm{E} \quad 05$ & 2.57 \\
\hline 5 & 0.1052 & 0.1226 & $4.18 \mathrm{E} \quad 05$ & 2.37 \\
\hline 6 & 0.1226 & 0.1452 & $3.94 E$ o5 & 1.99 \\
\hline 7 & 0.1452 & 0.1695 & $2.97 \mathrm{E} \quad 05$ & 2.65 \\
\hline 8 & 0.1695 & 0.2008 & $2.49 \mathrm{E} \quad 05$ & 2.50 \\
\hline \multicolumn{5}{|c|}{ Run 1537A } \\
\hline 1 & 0.1447 & 0.1706 & $2.91 \mathrm{E} \quad 05$ & 0.98 \\
\hline 2 & 0.1706 & 0.2008 & $2.38 E \quad 05$ & 1.11 \\
\hline 3 & 0.2008 & 0.2354 & $1.99 \mathrm{E} \quad 05$ & 1.25 \\
\hline 4 & 0.2354 & 0.2743 & $1.70 \mathrm{E} \quad 05$ & 1.38 \\
\hline 5 & 0.2743 & 0.3218 & $1.45 \mathrm{~F}, 05$ & 1.39 \\
\hline 6 & 0.3218 & 0.3822 & $1.01 E 05$ & 1.62 \\
\hline 7 & 0.3822 & 0.4470 & $6.59 \mathrm{E} \quad 04$ & 2.54 \\
\hline 8 & 0.4470 & 0.5291 & $6.48 \mathrm{E} \quad 04$ & 2.11 \\
\hline \multicolumn{5}{|c|}{ Run $1536 \mathrm{~A}$} \\
\hline 1 & 0.3793 & 0.4448 & $7.57 \mathrm{E} \quad 04$ & 0.87 \\
\hline 2 & 0.4448 & 0.5291 & $6.41 \mathrm{E} \quad 04$ & 0.84 \\
\hline 3 & 0.5291 & 0.6227 & $6.04 \mathrm{E} \quad 04$ & 0.85 \\
\hline 4 & 0.6227 & 0.7258 & $5.06 \mathrm{E} \quad 04$ & 0.95 \\
\hline 5 & 0.7258 & 0.8569 & $3.29 E \quad 04$ & 1.14 \\
\hline 6 & 0.8569 & 1.0067 & $1.96 \mathrm{E} 04$ & 1.74 \\
\hline 7 & 1.0067 & 1.1846 & $1.28 \mathrm{E} \quad 04$ & 2.35 \\
\hline 8 & 1.1846 & 1.4000 & $1.03 \mathrm{E} \quad 04$ & 2.49 \\
\hline
\end{tabular}


Table 14. Bonner ball measurements on centerline at NE-213 location (I tems IA, IID, IVC, VIA, F)

\begin{tabular}{|c|c|c|c|c|c|c|c|c|}
\hline \multirow{3}{*}{ Configuration ${ }^{A}$} & \multicolumn{8}{|c|}{ Bonner ball count rates $\left(\mathrm{s}^{-1} \mathrm{w}^{-1}\right)$} \\
\hline & \multicolumn{2}{|c|}{ 3-inch Diam Ball } & \multicolumn{2}{|c|}{ 4-inch Diam Bal? } & \multicolumn{2}{|c|}{ 5-inch Diam Ball } & \multicolumn{2}{|c|}{ 10-inch Diam Ball } \\
\hline & Foreground $b$ & Background $\mathbf{C}$ & Foreground & Background & Foreground & Background & Foreground & Background \\
\hline IA & $9.21(1)^{\mathrm{d}}$ & 2.35 (1) & & & $3.67 \quad(2)$ & $5.43(1)$ & $1.31 \quad(2)$ & $1.12(1)$ \\
\hline I ID & $6.68(-3)$ & & & & $4.10(-2)$ & & $2.07(-2)$ & \\
\hline IVE; & $3,36(-2)$ & & & & $1.76(-1)$ & & $6.95 \quad(-2)$ & \\
\hline VऽF & $4.51 \quad(-3)$ & & & & $2.22(-2)$ & & $1.12(-2)$ & \\
\hline
\end{tabular}

a See experimental program plan in Appendix A for description of configurations.

beutron flux without shadow shield between detector and configuration.

Neutron flux with shadow shield between detector and configuration.

Read: $9.21 \times 10^{1}$ 
Table 15. Bonner ball measurements on centerline at $30 \mathrm{~cm}$ behind a series of configurations (Items IA, IIA-E, IIIA-E, IVA-I)

\begin{tabular}{|c|c|c|c|c|c|c|}
\hline \multirow[b]{2}{*}{ Conf iguration ${ }^{a}$} & \multicolumn{6}{|c|}{ Bonner ball count rates $\left(s^{-1} W^{-1}\right)$} \\
\hline & $\begin{array}{l}\text { bare } \\
\text { Detector }\end{array}$ & $\begin{array}{l}\text { CD-covered } \\
\text { Detector }\end{array}$ & $\begin{array}{c}3-\text { inch } \\
\text { diam ball }\end{array}$ & $\begin{array}{c}5 \text {-inch } \\
\text { diam ball }\end{array}$ & $\begin{array}{c}8 \text {-inch } \\
\text { diam ball }\end{array}$ & $\begin{array}{c}10 \text {-inch } \\
\text { diam ball }\end{array}$ \\
\hline IA & & & $5.92(2)^{b}$ & $2.86 \quad(3)$ & $2.10(3)$ & $1.13(3)$ \\
\hline I I A & & & $2.00(2)$ & $8.31(2)$ & $5.68(2)$ & $2.71(2)$ \\
\hline I IH & & & $4.35(0)$ & $2.64(1)$ & $2.04(1)$ & $1.07(1)$ \\
\hline I IC & & & $1.78(-1)$ & $1.26(0)$ & $1.02(0)$ & $5.76(-1)$ \\
\hline I ID & & & $1.03(-2)$ & $7.16(-2)$ & $6.68(-2)$ & $3.89(-2)$ \\
\hline I IE & $1.30(-3)$ & $1.53(-4)$ & $5.20(-3)$ & $2.47(-2)$ & $1.85(-2)$ & $9.63(-3)$ \\
\hline I IF: & $2.52(-4)^{\mathrm{C}}$ & & & & & \\
\hline I I IA & & & $2.88 \quad(2)$ & $1.33(3)$ & $9.17(2)$ & $4.59 \quad(2)$ \\
\hline I I I $B$ & & & $7.26(1)$ & $4.12(2)$ & 3.17 (2) & $1.63 \quad(2)$ \\
\hline IIIC: & & & $1.55 \quad(2)$ & $6.26 \quad(2)$ & $4.2 .4 \quad(2)$ & $2.13 \quad(2)$ \\
\hline I I I & & & 3.49 (1) & $2.02 \quad(2)$ & $1.47(2)$ & 7.36 (1) \\
\hline I I I $E$ & & & $1.07 \quad(1)$ & 6.13 (1) & 4.57 (1) & $2.37 \quad(1)$ \\
\hline IVA & & & $1.06(2)$ & $2.45 \quad(2)$ & $1.25 \quad(2)$ & $5.42(1)$ \\
\hline I VB & & & 2.40 (1) & 4.42 (1) & 1.87 (1) & $7.94 \quad(0)$ \\
\hline IVC & & & $5.09(2)$ & $2.98(-1)$ & $2.15(-1)$ & $1.16(-1)$ \\
\hline IVD & & & $3.11(-2)$ & $1.64(-1)$ & $1.21(-1)$ & $6.35(-2)$ \\
\hline IVE & & & $1.91(-2)$ & $4.93(-2)$ & $2.64(-2)$ & $1.20(-2)$ \\
\hline IVF & & & $5.43(-3)$ & $1.08(-2)$ & $4.82(-3)$ & $2.10(-3)$ \\
\hline IVG & & & $1.26(-3)$ & $2.20(-3)$ & $9.34(-4)$ & $3.95(-4)$ \\
\hline IVH & & & $7.15(-4)$ & $1.32(-3)$ & $5.83(-4)$ & $2.48(-4)$ \\
\hline IVI & & & $5.07(-4)$ & $8.95(-4)$ & $3.73(-4)$ & $1.55(-4)$ \\
\hline
\end{tabular}

${ }^{a}$ See experimental program plan on Appendix A for description of configurations.

$\mathrm{b}_{\text {Read: }} 5.92 \times 10^{2}$

${ }^{c}$ Cadmium enclosure that surrounded the detector exrept on the side of the last slab in the configuration. 
Table 16. Bonner ball measurements on centerline at $304.8 \mathrm{~cm}$ or $457.2 \mathrm{~cm}$ from the center of the reactor (Items IA, IIA-E, IIIA-E, IVA-F)

\begin{tabular}{|c|c|c|c|c|c|c|c|c|c|c|c|c|c|}
\hline \multirow{3}{*}{ configuration ${ }^{a}$} & \multirow{3}{*}{$\begin{array}{l}\text { Locationb } \\
(\mathbf{c o s})\end{array}$} & \multicolumn{12}{|c|}{ Bonner ball count: rates (s ${ }^{2} w^{1}$ ) } \\
\hline & & \multicolumn{2}{|c|}{ Bare Counter } & \multicolumn{2}{|c|}{ CD-Covered Counter } & \multicolumn{2}{|c|}{ 3-inch Dian Ball } & \multicolumn{2}{|c|}{ 5. inch Diam Bat1 } & \multicolumn{2}{|c|}{ 8-inch Dian Ball } & \multicolumn{2}{|c|}{ 10-1nch Diam Ball } \\
\hline & & Foreground ${ }^{c}$ & Background ${ }^{d}$ & Foreground & Background & Foreground & Background & Foreground & Background & Foreground & Background & Foreground & Background \\
\hline IA & 457.2 & & & & & $3.18(1)^{\mathrm{e}}$ & 1.06 (1) & $1.41(2)$ & 2.77 (1) & $9.49(1)$ & $1.44(1)$ & 4. 76 (1) & $6.77(0)$ \\
\hline IA & 304.8 & & & & & $\begin{array}{l}9.28 \text { (1) } \\
3.77(1)\end{array}$ & $\begin{array}{l}2.17 \text { (1) } \\
5.16(0)\end{array}$ & $4.16(2)$ & $5.33(1)$ & 2.93 (2) & 2.85 (11 & 1.45 (2) & 1.32 (1) \\
\hline IIA & 304.8 & & & & & $\begin{array}{l}3.77(1) \\
1.23(0)\end{array}$ & $\begin{array}{l}5.16(0) \\
1.54(-1)\end{array}$ & $\begin{array}{l}1.47(2) \\
6.35(0)\end{array}$ & $\begin{array}{l}1.23(1) \\
4.08(1)\end{array}$ & $\begin{array}{l}9.81(1) \\
4.84(0)\end{array}$ & $\begin{array}{l}6.30(0) \\
2.29 \\
2-1\}\end{array}$ & $\begin{array}{l}4.67(1) \\
2.55 \\
2.50)\end{array}$ & $\begin{array}{l}2.83(0) \\
1.09(-1) \\
1.09\end{array}$ \\
\hline IIB & 304.8 & & & & & $6.13(-2)$ & $\begin{array}{l}1.54(-1) \\
7.76(-3)\end{array}$ & $\begin{array}{l}6.35 \\
4.03(-1)\end{array}$ & $\begin{array}{l}4.08(-1) \\
2.15(-2)\end{array}$ & $\begin{array}{l}4.84(0) \\
3.29(-1)\end{array}$ & $\begin{array}{l}2.29(-1) \\
1.20(-2)\end{array}$ & $\begin{array}{l}2.55(0) \\
1.87(-1)\end{array}$ & $\begin{array}{l}1.09(-1) \\
6.12(-3)\end{array}$ \\
\hline IIC & $\begin{array}{l}304.8 \\
304.8\end{array}$ & & & & & $5.04\{(-3\}$ & $7.80(-4)$ & $3.17(-2)$ & $1.85(-3)$ & $2.95(-2)$ & $1.13(-3)$ & $1.71(-2)$ & $5.71(-4)$ \\
\hline $\begin{array}{l}\operatorname{IID}_{11 \mathrm{E}} \\
\mathrm{In}\end{array}$ & $\begin{array}{l}304.8 \\
304.8\end{array}$ & $1.38(-3)$ & $1.00(-3)$ & $1.44(-4)$ & $6.06 \quad(-5)$ & $4.02(-3)$ & $4.87(-4)$ & $1.84(-2)$ & $9.75(-4)$ & $1.37(-2)$ & $4.82(-4)$ & $7.11(-3)$ & $2.25(-4)$ \\
\hline $\mathrm{HE}^{f}$ & $\begin{array}{l}304.8 \\
330.8\end{array}$ & 1.17 $17-3)$ & & & & $5.22(1)$ & & & & & & & \\
\hline 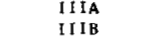 & $\begin{array}{l}304.8 \\
304.8\end{array}$ & & & & & 1.50 (11) & $2.42(0)$ & $7.82(1)$ & $6.65(0)$ & $5.82(1)$ & $3.63(0)$ & 2.86 (1) & $1.70(0)$ \\
\hline 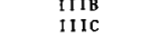 & $\begin{array}{l}304.8 \\
304.8\end{array}$ & & & & & $3.07(11)$ & $4.28(0)$ & $\begin{array}{l}18(2) \\
1.18(2)\end{array}$ & $1.03(1)$ & 7.87 (1) & $5.22(0)$ & $3.86(1)$ & $2.32(0)$ \\
\hline III & 304.8 & & & & & $7.96(0)$ & $1.04(0)$ & $4.18(1)$ & $2.84(0)$ & 3.11 (1) & $1.67(0)$ & 1.52 (1) & $7.52\{-1\}$ \\
\hline IIIE & 304.8 & & & & & $2.68(0)$ & $4.23(-1)$ & $1.47(1)$ & $1.14(0)$ & $1.06(1)$ & $5.75(-1)$ & $5.54(0)$ & 2. $68(-1)$ \\
\hline IVA & 304.8 & & & & & $2.31(1)$ & $2.43(0)$ & $5.28(1)$ & $4.27(0)$ & $2.63(1)$ & $1.80(0)$ & 1. $12(1)$ & $7.47(-1)$ \\
\hline IVB & $\begin{array}{l}304.8 \\
304.8\end{array}$ & & & & & $\begin{array}{l}6.34(0) \\
2.04\end{array}$ & $\begin{aligned} 3.36 & (-1) \\
2.28 & (-3)\end{aligned}$ & $\begin{array}{l}1.16(1) \\
1.13(-1)\end{array}$ & $\begin{array}{l}5.56(-1) \\
5.50(-3)\end{array}$ & $\begin{array}{l}4.93(0) \\
8.19^{(02)}\end{array}$ & $\begin{array}{l}2.07(-1) \\
2.84(-3)\end{array}$ & $\begin{array}{l}2.06(0) \\
4.49(-1)\end{array}$ & $8.75(-2)$ \\
\hline Twe & $\begin{array}{l}304.8 \\
457.2\end{array}$ & & & & & $\begin{array}{l}2.04(-2) \\
5.56(-3)\end{array}$ & $\begin{array}{l}2.28 \\
1.84(-3) \\
1.34\end{array}$ & $\begin{array}{l}1.13(-1) \\
2.66(-2)\end{array}$ & $\begin{array}{l}5.50(-3) \\
4.51(-3)\end{array}$ & $\begin{array}{l}8.19(-2) \\
1.91(-2)\end{array}$ & $\begin{array}{l}2.84(-3) \\
2.42(-3)\end{array}$ & $\begin{array}{l}4.49(-1) \\
1.03(-2)\end{array}$ & $\begin{array}{l}1.41(-3) \\
1.18(-3)\end{array}$ \\
\hline $\begin{array}{l}\text { IV } \\
\text { IVD }\end{array}$ & $\begin{array}{l}\begin{array}{l}457.2 \\
457.2\end{array} \\
457\end{array}$ & & & & & $\begin{array}{l}5.56(-3) \\
3.69(-3)\end{array}$ & $\begin{array}{l}1.84(-3) \\
1.33(-3)\end{array}$ & $\begin{array}{l}2,66 \\
1.54(-2)\}\end{array}$ & $3.06(-3)$ & $\left.\begin{array}{l}1.91(1-2) \\
1.10\end{array}-2\right\}$ & $1.60(-3)$ & $5.67(-3)$ & $7.30(-4)$ \\
\hline IVE & 457.2 & & & & & $\begin{array}{l}2.53(-3) \\
1.21(-3)\end{array}$ & $\begin{array}{l}8.50(-1) \\
7.04 \\
(-4)\end{array}$ & $\begin{array}{l}5.89(-3) \\
2.41(-3)\end{array}$ & $\begin{array}{l}1.63(-3) \\
1.34 \\
(-3)\end{array}$ & $\begin{array}{l}3.09(-3) \\
1.08(-3)\end{array}$ & $\begin{array}{l}7.40(-4) \\
6.08(-4)\end{array}$ & $\begin{array}{l}1.39(-3) \\
4.64(-4)\end{array}$ & $\begin{array}{l}3.40(-4) \\
2.70(-4)\end{array}$ \\
\hline IVF & 457.2 & & & & & $1.21(-3)$ & $7.04(-4)$ & $2.41(-3)$ & $1.34(-3)$ & $1.08(-3)$ & $0.08(-4)$ & $4.64(-4)$ & $2.70(-4)$ \\
\hline
\end{tabular}

experintal program plan in Appendix A for description of configurations.

Distance from center of reactor to center of detector.

Neut ron flux without shadow shield between detector and consuguration.

Pead: $3.18 \times 10^{1}$. 
Table 17. 5-inch Bonner Ball horizontal traverses through

midplane at $30 \mathrm{~cm}$ behind a series of configurations

(Items IA, IIA, D, E, IIIA, B, C, E)

\begin{tabular}{|c|c|c|c|c|c|c|c|c|c|c|c|c|}
\hline \multirow{2}{*}{$\begin{array}{c}\text { Distanre from } \\
\text { centerline } \\
(\mathrm{cm})\end{array}$} & \multicolumn{12}{|c|}{ Bonner Ball count rates $\left(\mathrm{s}^{-1} \mathrm{~W}^{-1}\right)$} \\
\hline & Item & $I A^{a}$ & Item & $\operatorname{II} A^{a}$ & Item & $\operatorname{IID} \mathrm{D}^{\mathrm{a}}$ & Item & $I I E^{a}$ & Item $111 A^{a}$ & Item $\operatorname{II} \mathrm{B}^{\mathrm{a}}$ & Item $111 \mathrm{IC}^{\mathrm{a}}$ & Item IIIE $\mathrm{E}^{\mathbf{a}}$ \\
\hline 76.25 & & & 2.85 & $(2)^{b}$ & 4.40 & $(-2)$ & 1.23 & $(-2)$ & $4.38 \quad(2)$ & 1.44 (2) & $2.20 \quad(2)$ & 2.29 (1) \\
\hline 70.3 & 1.30 & (3) & & & & & & & & & & \\
\hline 60 & 1.56 & (3) & 4.31 & (2) & 4.00 & $(-2)$ & 1.56 & $(-2)$ & $6.71(2)$ & $2.19(2)$ & $3.35(2)$ & $3.40(1)$ \\
\hline 45 & 2.03 & (3) & 5.76 & (2) & 5.28 & $(-2)$ & 1. . 90 & $(-2)$ & $8.99(2)$ & $2.88(2)$ & 4.43 (2) & 4.49 (1) \\
\hline 30 & 2.46 & $(3)$ & 7.07 & (2) & 6.33 & $(-2)$ & 2.19 & $(-2)$ & $1.10 \quad(3)$ & 3.51 (2) & 5.42 (2) & 5.48 (1) \\
\hline 15 & 2.68 & (3) & 8.15 & (2) & 6.91 & $(-2)$ & 2.44 & $(-2)$ & 1.17 (3) & 3.95 (2) & $6.06 \quad(2)$ & 6.00 (1) \\
\hline 0 & 2.89 & (3) & 8.29 & (2) & 7.21 & $(-2)$ & 2.48 & $(-2)$ & $1.28 \quad(3)$ & 4.14 (2) & $6.29(2)$ & 6.19 (1) \\
\hline 15 & 2.77 & (3) & 7.94 & (2) & 7.03 & $(-2)$ & 2.44 & $(-2)$ & 1.24 (3) & 3.94 (2) & 6.03 (2) & 5.96 (1) \\
\hline 30 & 2.38 & (3) & 7.16 & (2) & 6.32 & $(-2)$ & 2.27 & $(-2)$ & 1.12 (3) & 3.49 (2) & 5.47 (2) & 5.27 (1) \\
\hline 45 & 1.95 & (3) & 5.73 & (2) & 5.23 & $(-2)$ & 1.99 & $(-2)$ & 8.87 (2) & $2.84 \quad(2)$ & 4.47 (2) & 4.30 (1) \\
\hline 60 & 1.46 & (3) & 4.23 & (2) & 4.04 & $(-2)$ & 1.61 & $(-2)$ & 6.72 (2) & 2.09 (2) & $3.50(2)$ & 3.22 (1) \\
\hline 75 & 1.03 & (3) & & & & & & & & & & \\
\hline $76.2 \mathrm{~N}$ & & & 2.74 & (2) & 3.09 & $(-2)$ & 1.23 & $(-2)$ & $4.46 \quad(2)$ & $1.35 \quad(2)$ & $2.31 \quad(2)$ & 2.14 (1) \\
\hline
\end{tabular}

a see experimental program plan in Appendix A for description of experiments.

Bead: $2.85 \times 10^{2}$ 
Table 18. Past neutron fluxes $(>0.8 \mathrm{MeV})$ on centerline at $47.3 \mathrm{~cm}$ behind the $\mathrm{B}_{4} \mathrm{C}(33.0 \mathrm{~cm}$ behind lead) (Item IID): Run 7857A

\begin{tabular}{|c|c|c|c|c|c|c|c|c|c|}
\hline \multicolumn{2}{|c|}{$\begin{array}{l}\text { Neutron } \\
\text { Energy } \\
(\mathrm{MeV})\end{array}$} & \multicolumn{3}{|c|}{$\begin{array}{l}\text { Flux (neutrons } \\
\text { Lower } \\
\text { Limit }\end{array}$} & \multicolumn{2}{|c|}{$\begin{array}{c}\left.\mathrm{cm}^{-2} \mathrm{MeV}^{-1} \mathrm{~kW}^{-1} \mathrm{~s}^{-1}\right) \\
\text { Upper } \\
\text { Limit }\end{array}$} & $\begin{array}{l}\text { Neutron } \\
\text { Energy } \\
(\mathrm{MeV})\end{array}$ & $\begin{array}{l}\text { Flux fneutrons } \\
\text { Lower } \\
\text { Limit }\end{array}$ & $\begin{array}{c}\left.\mathrm{cm}^{-2} \mathrm{MeV}^{-1} \mathrm{~kW}^{-1} \mathrm{~s}^{-1}\right) \\
\text { Upper } \\
\text { Limit }\end{array}$ \\
\hline $8.11 \mathrm{E}$ & $(-1)$ & & $8.75 \mathrm{E}$ & $(0)$ & $8.81 \mathrm{E}$ & $(0)$ & $5.94 \mathrm{E}(0)$ & 4. $46 \mathrm{E} \quad(-2)$ & $4.73 E(-2)$ \\
\hline $9.07 E$ & $(-1)$ & & $8.61 E$ & $(0)$ & $8.64 E$ & $(0)$ & $6.25 E(0)$ & $4.03 E \quad(-2)$ & $4.38 E(-2)$ \\
\hline $1.01 \mathrm{E}$ & $(0)$ & & $6.25 \mathrm{E}$ & $(0)$ & $6.27 \mathrm{E}$ & $(0)$ & $6.56 \mathrm{E}(0)$ & $3.73 E(-2)$ & 4.02E $(-2)$ \\
\hline $1.11 \mathrm{E}$ & $(0)$ & & $3.96 \mathrm{E}$ & $(0)$ & $3.98 \mathrm{E}$ & $(0)$ & $6.84 \mathrm{E}(0)$ & $3.40 E(-2)$ & $3.61 \mathrm{E}(-2)$ \\
\hline $1.20 \mathrm{E}$ & $(0)$ & & $2.61 \mathrm{E}$ & (0) & $2.63 \mathrm{E}$ & (0) & $7.24 \mathrm{E}(0)$ & $2.90 \mathrm{~F} \quad(-2)$ & $3.08 \mathrm{E}(-2)$ \\
\hline $1.31 \mathrm{E}$ & $(0)$ & & $1.95 E$ & $(0)$ & $1.96 \mathrm{E}$ & $(0)$ & $7.74 \mathrm{E}(0)$ & $2.44 E \quad(-2)$ & 2.63E $(-2)$ \\
\hline $1.41 \mathrm{E}$ & $(0)$ & & $1.68 \mathrm{E}$ & $(0)$ & $1.69 \mathrm{E}$ & $(0)$ & $8.24 E(0)$ & $2.01 \mathrm{E}(-2)$ & $2.18 \mathrm{E}(-2)$ \\
\hline $1.51 \mathrm{E}$ & $(0)$ & & $1.52 \mathrm{E}$ & $(0)$ & $1.54 \mathrm{E}$ & (0) & $8.76 \mathrm{E} \quad(0)$ & $1.54 \mathrm{E} \quad(-2)$ & $1.65 \mathrm{E}(-2)$ \\
\hline $1.61 \mathrm{E}$ & (0) & & $1.39 \mathrm{E}$ & (0) & $1.40 \mathrm{E}$ & (0) & $9.26 \mathrm{E} \quad(0)$ & 1.23E $(-2)$ & 1. $33 E(-2)$ \\
\hline $1.71 \mathrm{E}$ & $(0)$ & & $1.27 \mathrm{E}$ & (0) & $1.28 \mathrm{E}$ & $(0)$ & $9.74 \mathrm{E} \quad(0)$ & $9.96 \mathrm{E}(-3)$ & $1.09 \mathrm{E}(-2)$ \\
\hline $1.81 E$ & (0) & & $1.15 \mathrm{E}$ & (0) & $1.16 \mathrm{E}$ & (0) & 1.03E (1) & $7.26 \mathrm{E}(-3)$ & $8.05 E(-3)$ \\
\hline $1.93 \mathrm{E}$ & $(0)$ & & $1.03 E$ & $(0)$ & $1.04 \mathrm{E}$ & $(0)$ & $1.08 E(1)$ & $5.32 E(-3)$ & $5.99 \hat{E} \quad(-3)$ \\
\hline $2.10 \mathrm{E}$ & $(0)$ & & $8.24 \mathrm{E}$ & $(-1)$ & $8.34 E$ & $(-1)$ & $1.12 \mathrm{E}$ (1) & $4.28 E(-3)$ & $4.89 \mathrm{E} \quad(-3)$ \\
\hline $2.30 \mathrm{E}$ & $(0)$ & & $5.98 \mathrm{E}$ & $(-1)$ & $6.07 \mathrm{E}$ & $(-1)$ & $1.18 \mathrm{E}(1)$ & $3.52 E(-3)$ & $4.05 E(-3)$ \\
\hline $2.50 \mathrm{E}$ & $(0)$ & & $4.45 \mathrm{E}$ & $(-1)$ & $4.53 E$ & $(-1)$ & $1.24 E(1)$ & $2.56 E(-3)$ & $3.01 E(-3)$ \\
\hline $2.70 \mathrm{E}$ & (0) & & $3.52 \mathrm{E}$ & $(-1)$ & $3.59 \mathrm{E}$ & $(-1)$ & $1.32 E$ (1) & 1. $26 \mathrm{E}(-3)$ & 1.62E $(-3)$ \\
\hline $2.90 \mathrm{E}$ & $(0)$ & & $2.98 \mathrm{E}$ & $(-1)$ & $3.05 \mathrm{E}$ & $(-1)$ & $1.40 \mathrm{E}(1)$ & $4.84 E(-4)$ & 8. $32 E(-4)$ \\
\hline $3.10 \mathrm{E}$ & $(0)$ & & $2.60 \mathrm{E}$ & $(-1)$ & $2.67 \mathrm{E}$ & $(-1)$ & $1.48 \mathrm{E}(1)$ & $4.70 E(-4)$ & $9.03 E(-4)$ \\
\hline $3.30 E$ & $(0)$ & & $2.29 E$ & $(-1)$ & $2.35 \mathbf{E}$ & $(-1)$ & $1.56 \mathrm{E} \quad(1)$ & $6.94 E(-4)$ & $1.10 E(-3)$ \\
\hline $3.50 \mathrm{E}$ & (0) & & $2.03 E$ & $(-1)$ & $2.09 \mathrm{E}$ & $(-1)$ & $1.65 \mathrm{E}$ (1) & $6.02 E(-4)$ & $9.65 \mathrm{E}(-4)$ \\
\hline $3.71 \mathrm{E}$ & (0) & & $1.80 \mathrm{E}$ & $(-1)$ & $1.85 \mathrm{E}$ & $(-1)$ & $1.75 \mathrm{E}$ (1) & $2.27 \mathrm{E}(-4)$ & $6.16 E(-4)$ \\
\hline $3.91 \mathrm{E}$ & $(0)$ & & $1.60 \mathrm{E}$ & $(-1)$ & $1.64 \mathrm{E}$ & $(-1)$ & $1.85 \mathrm{E}$ (1) & $-1.05 E \quad(-4)$ & $3.53 E(-4)$ \\
\hline $4.15 \mathrm{E}$ & $(0)$ & & 1. $36 \mathrm{E}$ & $(-1)$ & $1.40 \mathrm{~F}$ & $(-1)$ & $1.95 \mathrm{E} \quad(1)$ & $-2.40 \mathrm{E}(-4)$ & $2.17 \mathrm{E}(-4)$ \\
\hline $4.45 E$ & (0) & & $1.06 \mathrm{E}$ & $(-1)$ & $1.10 \mathrm{E}$ & $(-1)$ & $2.06 \mathrm{E}$ (1) & $-3.00 \mathrm{E}(-4)$ & 1. $57 \mathrm{E}(-4)$ \\
\hline $4.75 \mathrm{E}$ & $(0)$ & & $8.10 \mathrm{E}$ & $(-2)$ & $8.43 \mathrm{~F}$ & $(-2)$ & $2.16 \mathrm{E} \quad(1)$ & $-2.16 \mathrm{E}(-4)$ & $2.26 \mathrm{E}(-4)$ \\
\hline $5.04 \mathrm{E}$ & $(0)$ & & $6.54 \mathrm{E}$ & $(-2)$ & $6.83 \mathrm{E}$ & $(-2)$ & $2.26 \mathrm{E}$ (1) & $-2.02 E \quad(-4)$ & $2.51 E(-4)$ \\
\hline $5.35 \mathbf{E}$ & $(0)$ & & $5.58 \mathrm{E}$ & $(-2)$ & $5.86 \mathrm{~F}$ & $(-2)$ & $2.35 \mathrm{E}$ (1) & $-1.95 \mathrm{E}(-4)$ & $2.97 \mathrm{E} \quad(-4)$ \\
\hline $5.65 \mathrm{E}$ & (0) & & $4.94 \mathrm{E}$ & $(-2)$ & $5.25 \mathrm{E}$ & $(-2)$ & & & \\
\hline
\end{tabular}

\begin{tabular}{rrrrr}
\hline $\begin{array}{c}\mathrm{EI} \\
(\mathrm{MeV})\end{array}$ & $\begin{array}{c}\mathrm{E2} \\
(\mathrm{MeV})\end{array}$ & $\begin{array}{c}\text { Integral } \\
\left.\text { (neutrons } \mathrm{cm}^{-2} \mathrm{~kW}^{-1} \mathrm{~s}^{-1}\right)\end{array}$ & $\begin{array}{c}\text { Error } \\
\left.\text { (neutrons } \mathrm{cm}^{-2} \mathrm{~kW}^{-1} \mathrm{~s}^{-1}\right)\end{array}$ \\
\hline 0.811 & 1.000 & $1.57 \mathrm{E}(0)$ & $2.96 \mathrm{E}(-3)$ \\
1.000 & 1.200 & $8.58 \mathrm{E}(-1)$ & $1.83 \mathrm{E}(-3)$ \\
1.200 & 1.600 & $7.22 \mathrm{E}(-1)$ & $2.86 \mathrm{E}(-3)$ \\
1.600 & 2.000 & $4.71 \mathrm{E}(-1)$ & $2.19 \mathrm{E}(-3)$ \\
2.000 & 3.000 & $5.08 \mathrm{E}(-1)$ & $3.96 \mathrm{E}(-3)$ \\
3.000 & 4.000 & $2.10 \mathrm{E}(-1)$ & $2.90 \mathrm{E}(-3)$ \\
4.000 & 6.000 & $1.61 \mathrm{E}(-1)$ & $3.22 \mathrm{E}(-3)$ \\
6.000 & 8.000 & $6.67 \mathrm{E}(-2)$ & $2.35 \mathrm{E}(-3)$ \\
8.000 & 10.000 & $3.00 \mathrm{E}(-2)$ & $1.20 \mathrm{E}(-3)$ \\
10.000 & 12.000 & $1.09 \mathrm{E}(-2)$ & $6.50 \mathrm{E}(-4)$ \\
12.000 & 16.000 & $5.17 \mathrm{E}(-3)$ & $8.01 \mathrm{E}(-4)$ \\
16.000 & 20.000 & $1.33 \mathrm{E}(-3)$ & $8.36 \mathrm{E}(-4)$ \\
1.500 & 15.000 & $1.61 \mathrm{E}(0)$ & $1.77 \mathrm{E}(-2)$ \\
3.000 & 12.000 & $4.78 \mathrm{E}(-1)$ & $1.03 \mathrm{E}(-2)$ \\
\hline
\end{tabular}


Table 19. Neutron fluxes (50 keV to $1.4 \mathrm{MeV}$ ) on centerline $47.3 \mathrm{~cm}$ beyond $\mathrm{B}_{4} \mathrm{C}$ ( $33.0 \mathrm{~cm}$ beyond $\mathrm{Pb}$ ) (Item IID): Runs 1540A, 1539B, 1539A

$\mathrm{N} \quad \begin{gathered}\text { Energy Boundary } \\ (\mathrm{MeV})\end{gathered}$

$$
\text { (Neutrons } \mathrm{cm}^{-\mathrm{Fl}} \mathrm{MeV}^{-1} \mathrm{kw}^{-1} \mathrm{~s}^{-1} \text { ) }
$$

Error

$(\%)$

Run 1540A

$\begin{array}{rll}1 & 0.0535 & 0.0626 \\ 2 & 0.0626 & 0.0735 \\ 3 & 0.0735 & 0.0862 \\ 4 & 0.0862 & 0.1025 \\ 5 & 0.1025 & 0.1207 \\ 6 & 0.1207 & 0.1406 \\ 7 & 0.1406 & 0.1661 \\ 8 & 0.1661 & 0.1951 \\ 9 & 0.1951 & 0.2296 \\ 10 & 0.2296 & 0.2713\end{array}$

$\begin{array}{lll}8.04 \mathrm{E} & 01 & 3.99 \\ 7.40 \mathrm{E} & 01 & 4.01 \\ 7.00 \mathrm{E} & 01 & 4.02 \\ 5.79 \mathrm{E} & 01 & 4.10 \\ 5.34 \mathrm{E} & 01 & 4.51 \\ 5.28 \mathrm{E} & 01 & 4.63 \\ 4.44 \mathrm{E} & 01 & 4.62 \\ 4.03 \mathrm{E} & 01 & 4.91 \\ 3.46 \mathrm{E} & 01 & 5.21 \\ 3.02 \mathrm{E} & 01 & 5.27\end{array}$

Run 1539B

$\begin{array}{lll}1 & 0.1938 & 0.2303 \\ 2 & 0.2303 & 0.2713 \\ 3 & 0.2713 & 0.3215 \\ 4 & 0.3215 & 0.3762 \\ 5 & 0.3762 & 0.4400 \\ 6 & 0.4400 & 0.5221\end{array}$

$\begin{array}{lll}3.33 \mathrm{E} & 01 & 2.07 \\ 2.98 \mathrm{E} & 01 & 2.30 \\ 2.70 \mathrm{E} & 01 & 2.27 \\ 2.29 \mathrm{E} & 01 & 2.73 \\ 1.82 \mathrm{E} & 01 & 3.23 \\ 1.91 \mathrm{E} & 01 & 2.49\end{array}$

Run $1539 \mathrm{~A}$

$\begin{array}{lll}1 & 0.3835 & 0.4482 \\ 2 & 0.4482 & 0.5221 \\ 3 & 0.5221 & 0.6145 \\ 4 & 0.6145 & 0.7254 \\ 5 & 0.7254 & 0.8548 \\ 6 & 0.8548 & 1.0119 \\ 7 & 1.0119 & 1.1875 \\ 8 & 1.1875 & 1.4000\end{array}$

$\begin{array}{lll}1.76 \mathrm{E} & 01 & 1.11 \\ 1.85 \mathrm{E} 01 & 1.01 \\ 1.80 \mathrm{E} 01 & 0.86 \\ 1.62 \mathrm{E} 01 & 0.82 \\ 1.31 \mathrm{E} 01 & 0.86 \\ 1.01 \mathrm{E} 01 & 0.86 \\ 5.20 \mathrm{E} 00 & 1.38 \\ 2.20 \mathrm{E} 00 & 2.55\end{array}$


Table 20. 5-inch Bonner Ball horizontal traverse through midplane at $30 \mathrm{~cm}$ behind a series of configurations (I tems IVA, C, G).

\begin{tabular}{|c|c|c|c|c|c|}
\hline \multirow{2}{*}{$\begin{array}{c}\text { Distance from } \\
\text { centerline } \\
(\mathrm{cm})\end{array}$} & \multicolumn{5}{|c|}{ Bonner Ball count rates $\left(s^{-1} W^{-1}\right)$} \\
\hline & Item IV-A & Item & IV $-C$ & Item & $I V-G^{a}$ \\
\hline $76.2 \mathrm{~s}$ & $8.57(1)^{b}$ & 1.05 & $(-1)$ & 1.07 & $(-3)$ \\
\hline 60 & 1.32 (2) & 1.56 & $(-1)$ & 1.38 & $(-3)$ \\
\hline 45 & 1.77 (2) & 2.06 & $(-1)$ & 1.68 & $(-3)$ \\
\hline 30 & $2,14 \quad(2)$ & 2.50 & $(-1)$ & 1.95 & $(-3)$ \\
\hline 15 & $2.43 \quad(2)$ & 2.72 & $(-1)$ & 2.06 & $(-3)$ \\
\hline 0 & $2.50 \quad(2)$ & 2.78 & $(-1)$ & 2.13 & $(-3)$ \\
\hline 15 & $2.40 \quad(2)$ & 2.63 & $(-1)$ & 2.08 & $(-3)$ \\
\hline 30 & 2.09 (2) & 2.31 & $(-1)$ & 1.93 & $(-3)$ \\
\hline 45 & $1.60 \quad(2)$ & 1.89 & $(-1)$ & 1.69 & $(-3)$ \\
\hline 60 & 1.25 (2) & 1.39 & $(-1)$ & 1.42 & $(-3)$ \\
\hline 76.2 & 8.10 (1) & 9.04 & $(-2)$ & 1,17 & $(-3)$ \\
\hline 91.2 & & & & 1.01 & $(-3)$ \\
\hline 106.2 & & & & 9.17 & $(-4)$ \\
\hline 121.2 & & & & 9.08 & $(-4)$ \\
\hline $136.2 \mathrm{~N}$ & & & & 9.23 & $(-4)$ \\
\hline
\end{tabular}

a See experimental program plan in Appendix $A$ for description of configurations .

$b_{\text {Read: }} 8.57 \times 10^{1}$. 
Table 21. Fast neutron fluxes $(>0.8 \mathrm{MeV})$ on centerline $42.8 \mathrm{~cm}$ beyond sS $(32.9 \mathrm{~cm}$ beyond $\mathrm{Pb}$ ) (Item IVC): Run 7860A.

\begin{tabular}{|c|c|c|c|c|c|c|c|c|c|}
\hline \multicolumn{2}{|c|}{$\begin{array}{l}\text { Neutron } \\
\text { Energy } \\
(\mathrm{MeV})\end{array}$} & \multirow{2}{*}{ Flux } & \multicolumn{2}{|c|}{$\begin{array}{l}\text { (neutrons } \\
\text { Lower } \\
\text { Limit }\end{array}$} & \multicolumn{2}{|c|}{$\begin{array}{c}\mathrm{cm}^{-2} \mathrm{MeV}^{-1} \mathrm{kw}^{-1} \mathrm{~s}^{-1} \text {, } \\
\text { Upper } \\
\text { I.imit }\end{array}$} & $\begin{array}{l}\text { Neutron } \\
\text { Energy } \\
(\mathrm{MeV})\end{array}$ & $\begin{array}{l}\text { Flux (neutrons } \\
\text { Lower } \\
\text { limit }\end{array}$ & \multirow{2}{*}{$\frac{\begin{array}{c}\mathrm{cm}^{-2} \mathrm{MeV}^{-1} \mathrm{~kW}^{-1} \mathrm{~s}^{-1} \\
\text { Upper } \\
\mathrm{Limit}\end{array}}{1.55 \mathrm{E}(-1)}$} \\
\hline $8.11 E$ & $(-1)$ & & $2.29 \mathrm{~F}$ & (1) & $2.31 \mathrm{~F}$ & (1) & $5.94 E \quad(0)$ & $1.46 \mathrm{~F}(1)$ & \\
\hline $9.07 \mathrm{E}$ & $(-1)$ & & $2.34 E$ & (1) & $2.35 \mathrm{E}$ & (1) & $6.25 E(0)$ & $1.34 E(-1)$ & $1.45 \mathrm{E}(-1)$ \\
\hline $1.01 \mathrm{E}$ & $(0)$ & & $1.81 \mathrm{E}$ & (1) & $1.81 \mathrm{E}$ & (1) & $6.56 \mathrm{~F} \quad(0)$ & 1. $16 \mathrm{~F}(-1)$ & $1.26 E(-1)$ \\
\hline $1.11 \mathrm{E}$ & $(0)$ & & $1.22 E$ & (1) & 1. $23 \mathrm{E}$ & (1) & $6.84 \mathrm{E}(0)$ & $9.92 E(-2)$ & $1.06 \mathrm{E}(-1)$ \\
\hline $1.20 \mathrm{E}$ & $(0)$ & & $8.40 F$ & $(0)$ & $8.45 \mathrm{E}$ & (0) & $7.24 \mathrm{E}(0)$ & $7.87 E(-2)$ & $8.46 \mathrm{E}(-2)$ \\
\hline $1.31 \mathrm{E}$ & $(0)$ & & $6.23 E$ & (0) & $6.28 E$ & (0) & $7.74 \mathrm{E}(0)$ & $6.13 E(-2)$ & $6.77 E(-2)$ \\
\hline $1.41 \mathrm{E}$ & (0) & & $5.19 \mathrm{~F}$ & $(0)$ & $5.24 \mathrm{E}$ & (0) & $8.24 \mathrm{E} \quad(0)$ & $5.30 \mathrm{E} \quad(-2)$ & $5.86 \mathrm{E},(-2)$ \\
\hline $1.51 \mathrm{E}$ & (0) & & $4.57 \mathrm{E}$ & (0) & $4.61 \mathrm{E}$ & (0) & $8.76 \mathrm{E} \quad(0)$ & $4.46 \mathrm{E}(-2)$ & $4.87 \mathrm{E}(-2)$ \\
\hline $1.61 \mathrm{E}$ & $(0)$ & & $4.03 \mathrm{E}$ & (0) & $4.06 \mathrm{~F}$ & $(0)$ & $9.26 \mathrm{E} \quad(0)$ & $3.44 E(-2)$ & $3.79 \mathrm{E}(-2)$ \\
\hline $1.7 \mathrm{tE}$ & (0) & & $3.52 E$ & (0) & $3.55 \mathrm{E}$ & (a) & $9.74 \mathrm{E}(0)$ & $2.69 E(-2)$ & $3.02 E(-2)$ \\
\hline $1.81 \mathrm{E}$ & $(0)$ & & $3.05 \mathrm{~F}$ & (0) & $3.08 \mathrm{E}$ & $(0)$ & $1.03 E$ (1) & $2.22 \mathrm{E} \quad(-2)$ & $2.51 E(-2)$ \\
\hline $1.93 \mathrm{E}$ & (0) & & $2.57 \mathrm{E}$ & (0) & $2.60 \mathrm{E}$ & (0) & $1.08 \mathrm{E}$ (1) & $1.90 \mathrm{E}(-2)$ & $2.15 E(-2)$ \\
\hline $2.10 \mathrm{E}$ & $(0)$ & & $1.98 \mathrm{E}$ & (0) & $2.00 \mathrm{E}$ & (0) & $1.12 \mathrm{E}$ (1) & $1.60 E(-2)$ & $1.83 E(-2)$ \\
\hline $2.30 \mathrm{E}$ & (0) & & $1.40 \mathrm{E}$ & (0) & $1.42 \mathrm{E}$ & (0) & $1.18 \mathrm{E}(1)$ & $1.31 E(-2)$ & 1.50E $(-2)$ \\
\hline $2.50 \mathrm{E}$ & (0) & & $9.68 \mathrm{E}$ & $(-1)$ & $9.88 \mathrm{E}$ & $(-1)$ & $1.24 \mathrm{E}$ & $9.50 \mathrm{E}(-3)$ & 1. $12 \mathrm{E}:(-2)$ \\
\hline $2.70 \mathrm{E}$ & (0) & & $7.16 \mathrm{E}$ & $(-1)$ & $7.37 \mathrm{E}$ & $(-1)$ & $1.32 E(1)$ & $5.71 E(-3)$ & $6.88 \mathrm{E}(-3)$ \\
\hline $2.90 \mathrm{~F}$ & $(0)$ & & $6.03 E$ & $(-1)$ & $6.23 \mathrm{E}$ & $(-1)$ & $1.40 \mathrm{E}$ (1) & $3,81 \mathrm{E}(-3)$ & $4.84 \mathrm{E} \quad(-3)$ \\
\hline $3.10 \mathrm{E}$ & $(0)$ & & $5.00 \mathrm{E}$ & $(-1)$ & $5.22 E$ & $(-1)$ & $1.48 \mathrm{E}(1)$ & $3.05 E(-3)$ & $3.81 E(-3)$ \\
\hline $3.30 \mathrm{E}$ & $(0)$ & & $3.84 \mathrm{E}$ & $(-1)$ & $4.01 \mathrm{E}$ & $(-1)$ & $1.56 \mathrm{E} \quad(1)$ & $1.98 E(-3)$ & $2.61 \mathrm{E}(-3)$ \\
\hline $3.50 \mathrm{E}$ & $(0)$ & & $3.17 \mathrm{E}$ & $(-1)$ & $3.37 \mathrm{E}$ & $(-1)$ & $1.65 \mathrm{E}(1)$ & $8.12 E(-4)$ & 1. $30 \mathrm{E}(-3)$ \\
\hline $3.71 \mathrm{E}$ & $(0)$ & & $3.10 \mathrm{E}$ & $(-1)$ & $3.26 \mathrm{E}$ & $(-1)$ & $1.75 E^{(1)}$ & $5.49 \mathrm{E} \quad(-5)$ & $5.05 \mathrm{E}(-4)$ \\
\hline $3.91 \mathrm{E}$ & (0) & & $3.12 \mathrm{E}$ & $(-1)$ & $3.25 \mathrm{E}$ & $(-1)$ & $1.85 \mathrm{E}(1)$ & $-2.57 \mathrm{E}(-4)$ & $2.19 E(-4)$ \\
\hline $4.15 \mathrm{E}$ & (0) & & $2.94 \mathrm{E}$ & $(-1)$ & $3.08 \mathrm{E}$ & $(-1)$ & $1.95 \mathrm{E}$ (1) & $-3.00 \mathrm{E}(-4)$ & $1.56 E(-4)$ \\
\hline $4.45 \mathrm{E}$ & (0) & & $2.54 \mathrm{E}$ & $(-1)$ & $2.65 \mathrm{E}$ & $(-1)$ & $2.06 \mathrm{E}(1)$ & $-3.02 E(-4)$ & $1.52 E(-4)$ \\
\hline $4.75 \mathrm{E}$ & (0) & & $2.18 \mathrm{E}$ & $(-1)$ & $2.29 \mathrm{E}$ & $(-1)$ & $2.16 \mathrm{E}(1)$ & $-2.10 \mathrm{E}(-4)$ & $2.28 \mathrm{E}(-4)$ \\
\hline $5.04 E$ & (0) & & $1.88 E$ & $(-1)$ & $1.98 \mathrm{E}$ & $(-1)$ & $2.26 \mathrm{E}$ (1) & $-2.04 \mathrm{E}(-4)$ & $2.46 E(-4)$ \\
\hline $5.35 \mathrm{E}$ & (0) & & $1.66 \mathrm{E}$ & $(-1)$ & $1.75 \mathrm{E}$ & $(-1)$ & $2.35 E(1)$ & $-2.03 E(-4)$ & $2.82 \mathrm{E}(-4)$ \\
\hline $5.65 \mathrm{E}$ & (0) & & $1.54 \mathrm{E}$ & $(-1)$ & $1.64 \mathrm{E}$ & $(-1)$ & & & \\
\hline
\end{tabular}

\begin{tabular}{rrrrr}
\hline $\begin{array}{c}\mathrm{E} 1 \\
(\mathrm{MeV})\end{array}$ & $\begin{array}{c}\mathrm{E} 2 \\
(\mathrm{MeV})\end{array}$ & $\begin{array}{c}\text { Integral } \\
\left.\text { (neutrons } \mathrm{cm}^{-2} \mathrm{~kW}^{-1} \mathrm{~s}^{-1}\right)\end{array}$ & $\begin{array}{c}\text { Error } \\
\left(\text { neutrons } \mathrm{cm}^{-2} \mathrm{~kW}^{-1} \mathrm{~s}^{-1}\right)\end{array}$ \\
\hline 0.811 & 1.000 & $4.26 \mathrm{E}(0)$ & $0.66 \mathrm{E}(-3)$ \\
1.000 & 1.200 & $2.60 \mathrm{E}(0)$ & $6.03 \mathrm{E}(-3)$ \\
1.200 & 1.600 & $2.25 \mathrm{E}(0)$ & $8.95 \mathrm{E}(-3)$ \\
1.600 & 2.000 & $1.26 \mathrm{E}(0)$ & $6.46 \mathrm{E}(-3)$ \\
2.000 & 3.000 & $1.15 \mathrm{E}(0)$ & $1.14 \mathrm{E}(-2)$ \\
3.000 & 4.000 & $3.73 \mathrm{E}(-1)$ & $8.82 \mathrm{E}(-3)$ \\
4.000 & 6.000 & $4.22 \mathrm{E}(-1)$ & $1.06 \mathrm{~F}(-2)$ \\
6.000 & 8.000 & $1.96 \mathrm{E}(-1)$ & $7.57 \mathrm{E}(-3)$ \\
8.000 & 10.000 & $8.37 \mathrm{E}(-2)$ & $4.09 \mathrm{E}(-3)$ \\
10.000 & 12.000 & $3.77 \mathrm{E}(-2)$ & $2.39 \mathrm{E}(-3)$ \\
12.000 & 16.000 & $2.14 \mathrm{E}(-2)$ & $2.10 \mathrm{~F}(-3)$ \\
16.000 & 20.000 & $1.37 \mathrm{E}(-3)$ & $9.43 \mathrm{E}(-4)$ \\
1.500 & 15.000 & $3.98 \mathrm{E}(0)$ & $5.51 \mathrm{E}(-2)$ \\
3.000 & 12.000 & $1.11 \mathrm{E}(0)$ & $3.35 \mathrm{E}(-2)$ \\
\hline
\end{tabular}


Table 22. Neutron fluxes (50 keV to $1.4 \mathrm{MeV})$ on

centerline $42.8 \mathrm{~cm}$ beyond $S S(32.9 \mathrm{~cm}$ beyond $\mathrm{Pb})$

(Iten IVC): Runs 1546A, 1546B, 1547A.

\begin{tabular}{|c|c|c|c|c|}
\hline $\mathrm{N}$ & \multicolumn{2}{|c|}{$\begin{array}{c}\text { Energy Boundary } \\
(\mathrm{MeV})\end{array}$} & (Neutron $\mathrm{cm}^{-\mathrm{F} l \mathrm{ux}} \mathrm{MeV}^{-1} \mathrm{~kW}^{-1} \mathrm{~s}^{-1}$ ) & \multirow[t]{2}{*}{$\begin{array}{l}\text { Error } \\
(\%)\end{array}$} \\
\hline \multicolumn{4}{|r|}{ Run 1546A } & \\
\hline 1 & 0.0542 & 0.0640 & $3.73 \mathrm{E} \quad 02$ & 3.08 \\
\hline 2 & 0.0640 & 0.0758 & $3.36 \mathrm{E} \quad 02$ & 3.13 \\
\hline 3 & 0.0758 & 0.0876 & $2.98 \mathrm{E} \quad 02$ & 3.91 \\
\hline 4 & 0.0876 & 0.1034 & $2.28 \mathrm{E} \quad 02$ & 4.08 \\
\hline 5 & 0.1034 & 0.1231 & $2.12 \mathrm{E} \quad 02$ & 3.81 \\
\hline 6 & 0,1231 & 0.1447 & $2.12 \mathrm{E} \quad 02$ & 3.82 \\
\hline 7 & 0.1447 & 0.1703 & $1.53 \mathrm{E} \quad 02$ & 4.80 \\
\hline \multicolumn{5}{|c|}{ Run $1546 \mathrm{~B}$} \\
\hline 1 & 0.1199 & 0.1451 & $2.08 \mathrm{E} \quad 02$ & 1.35 \\
\hline 2 & 0.1451 & 0.1703 & $1.46 \mathrm{E} \quad 02$ & 2.14 \\
\hline 3 & 0,1703 & 0.1998 & $1.31 \mathrm{E} 02$ & 2.26 \\
\hline 4 & 0.1998 & 0.2334 & $1.09 \mathrm{E} \quad 02$ & 2.58 \\
\hline 5 & 0.2334 & 0.2755 & $9.69 \mathrm{E} \quad 01$ & 2.50 \\
\hline 6 & 0.2755 & 0.3218 & $8.99 \mathrm{E} \quad 01$ & 2.70 \\
\hline 7 & 0.3218 & 0.3807 & $7.08 \mathrm{E} \quad 01$ & 2.84 \\
\hline 8 & 0.3807 & 0.4480 & $5.24 \mathrm{E} \quad 01$ & 3.68 \\
\hline 9 & 0.4480 & 0.5279 & $5.65 \mathrm{E} \quad 01$ & 3.06 \\
\hline \multicolumn{5}{|c|}{ Run $1547 \mathrm{~A}$} \\
\hline 1 & 0.3810 & 0.4452 & $5.23 E 01$ & 1.61 \\
\hline 2 & 0.4452 & 0.5279 & $5.48 \mathrm{E} \quad 01$ & 1.27 \\
\hline 3 & 0.5279 & 0.6197 & $5.13 \mathrm{E} \quad 01$ & 1.29 \\
\hline 4 & 0.6197 & 0.7298 & $4.46 E \quad 01$ & 1.24 \\
\hline 5 & 0.7298 & 0.8584 & $3.29 \mathrm{E} \quad 01$ & 1.41 \\
\hline 6 & 0.8584 & 1.0052 & $2.53 \mathrm{E} 01$ & 1.53 \\
\hline 7 & 1.0052 & 1.1889 & $1.42 \mathrm{E} \quad 01$ & 1.94 \\
\hline 8 & 1.1889 & 1.4000 & $6.38 \mathrm{E} 00$ & 3.54 \\
\hline
\end{tabular}


Table 23. Bonner Ball measurements on centerline behind a series of configurations (Items IVF - I).

\begin{tabular}{|c|c|c|c|c|c|c|c|c|c|}
\hline \multirow{3}{*}{ Configuration ${ }^{a}$} & \multirow{3}{*}{$\begin{array}{l}\text { Location } \\
\quad(\mathrm{cm})\end{array}$} & \multicolumn{8}{|c|}{ Bonner Ball count rates $\left(s^{-1} w^{-1}\right)$} \\
\hline & & \multicolumn{2}{|c|}{ 3-inch Dian Ball } & \multicolumn{2}{|c|}{ 5-inch Diam Ball } & \multicolumn{2}{|c|}{ 8-inch Diam Ball } & \multicolumn{2}{|c|}{ 10-inch Diam Ball } \\
\hline & & Foreground $^{c}$ & Background ${ }^{\mathrm{a}}$ & Foreground & Background & Foreground & Background & Foreground & Background \\
\hline IVF & 72.4 & $2.77(-3)$ & $3.60(-4)$ & $5.37(-3)$ & $6.50(-4)$ & $2.48(-3)$ & $2.83(-4)$ & $1.06(-3)$ & $1.19(-4)$ \\
\hline IVG & 53.6 & $1.02(-3)$ & $3.53(-4)$ & $1.79(-3)$ & $6.54(-4)$ & $7.65(-4)$ & $2.75(-4)$ & $3.23(-4)$ & $1.20(-4)$ \\
\hline IVH & 52.1 & $6.54(-4)$ & $3.59(-4)$ & $1.20(-3)$ & $6.43(-4)$ & $5.32(-4)$ & $2.84(-4)$ & $2.25(-4)$ & $1.21(-4)$ \\
\hline IVI & 49.5 & $5.03(-4)$ & $3.51(-4)$ & $9.30(-4)$ & $6.39(-4)$ & $3.93(-4)$ & 2. $73(-4)$ & $1.64(-4)$ & $1.17(-4)$ \\
\hline
\end{tabular}

${ }^{a}$ See experimental program plan in Appendix A for description of configurations.

$b_{1) i s t a n c e}$ from center of detector to back of configuration.

deutron flux without shadow shield between detector and configuration.

$d_{\text {Neutron }}$ flux with 12-inch LiH slab between detector and configuration.
Read: $2.77 \times 10^{-3}$. 
Table 24. Bonner Ball measurements on centerline midway in the void ${ }^{a}$ between Na tanks (Item IVI - M).

\begin{tabular}{|c|c|c|c|c|c|c|c|}
\hline \multirow[b]{2}{*}{ Configuration ${ }^{b}$} & \multicolumn{7}{|c|}{ Bonner Ball count } \\
\hline & $\begin{array}{c}\text { bare } \\
\text { detector }\end{array}$ & $\begin{array}{l}\text { CD-covered } \\
\text { detector }\end{array}$ & $\begin{array}{c}3 \text {-inch } \\
\text { diam ball }\end{array}$ & \multicolumn{2}{|c|}{$\begin{array}{c}\text { 5-inch } \\
\text { diam ball }\end{array}$} & $\begin{array}{c}\text { 8-inch } \\
\text { diam ball }\end{array}$ & $\begin{array}{c}\text { 10-inch } \\
\text { diam ball }\end{array}$ \\
\hline IVI & $6.54(-5)^{\mathrm{C}}$ & $2.85(-5)$ & $3.12(-4)$ & 5.40 & $(-4)$ & $2.11(-4)$ & $8.82(-5)$ \\
\hline IVJ & $2.92(-5)$ & $1.12(-5)$ & $1.14(-4)$ & 1.82 & $(-4)$ & $6.60(-5)$ & $2.48(-5)$ \\
\hline IVK & $1.38(-5)$ & $4.85(-6)$ & $4.83(-5)$ & 7.04 & $(-5)$ & $2.40(-5)$ & $9.34(-6)$ \\
\hline IVL & $5.95(-6)$ & $1.92(-6)$ & $1.76(-5)$ & 2.51 & $(-5)$ & $8.20(-6)$ & $3.10(-6)$ \\
\hline & $6.40(-6)$ & & $1.76(-5)$ & 2.48 & $(-5)$ & & $3.14(-6)$ \\
\hline IVM & $3.00(-6)$ & $7.34(-7)$ & $6.60(-6)$ & 8.50 & $(-6)$ & $2.66(-6)$ & $1.04(-6)$ \\
\hline
\end{tabular}

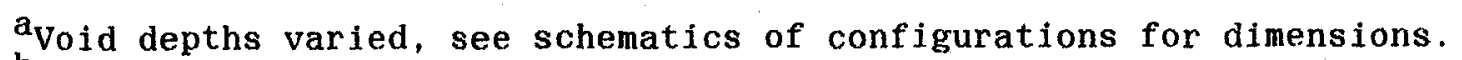

bee experimental program plan in Appendix A for description of configuration.

Read: $6.54 \times 10^{-5}$ 
Table 25. 5-inch Bonner ball traverses through horizontal midplane $30 \mathrm{~cm}$ behind a series of configurations (Itens VA, B, D, G, J).

\begin{tabular}{|c|c|c|c|c|c|c|}
\hline \multirow{2}{*}{$\begin{array}{l}\text { Distance from } \\
\text { centerline } \\
(\mathrm{cm})\end{array}$} & \multicolumn{6}{|c|}{ Bonner Ball count rates $\left(s^{-1} W^{-1}\right)$} \\
\hline & Item $\mathrm{VA}^{\mathrm{a}}$ & Item $\mathrm{VB}^{\mathrm{a}}$ & Item & $V^{a}$ & Item $V_{G}{ }^{a}$ & Item $\mathrm{VJ}^{\mathrm{a}}$ \\
\hline $77.5 \mathrm{~s}$ & & $7.84(0)^{b}$ & 1.27 & $(0)$ & $7.47 \quad(-2)$ & \\
\hline $76.2 \mathrm{~s}$ & & & & & & $2.32(-2)$ \\
\hline 75 & 2.69 (1) & & & & & \\
\hline 61.3 & & $1.19(1)$ & 1.78 & $(0)$ & & \\
\hline 60 & $3.55 \quad(1)$ & & & & $1.05(-1)$ & $3.19(-2)$ \\
\hline 46.3 & & $1.53(1)$ & 2.20 & $(0)$ & & \\
\hline 45 & 4.41 (1) & & & & $1.29(-1)$ & $3.93(-2)$ \\
\hline 31.3 & & 1.82 (1) & 2.55 & $(0)$ & & \\
\hline 30 & 5.04 (1) & & & & $1.44(-1)$ & $4.48 \quad(-2)$ \\
\hline 16.3 & & $1.98(1)$ & 2.78 & $(0)$ & & \\
\hline 15 & 5.42 (1) & & & & $1.55(-1)$ & $4.85(-2)$ \\
\hline 1.3 & & $2.03(1)$ & 2.86 & $(0)$ & & \\
\hline 0 & 5.62 (1) & & & & $1.61(-1)$ & $4.96(-2)$ \\
\hline 13.7 & & $1.99(1)$ & 2.80 & $(0)$ & & \\
\hline 15 & $5.49(1)$ & & & & $1.56(-1)$ & $4.81 \quad(-2)$ \\
\hline 28.7 & & 1.82 (1) & 2.60 & $(0)$ & & \\
\hline 30 & $5.13(1)$ & & & & $1.43(-1)$ & $4.43 \quad(-2)$ \\
\hline 43.7 & & $1.53(1)$ & 2.27 & $(0)$ & & \\
\hline 45 & $4.47 \quad(1)$ & & & & $1.25(-1)$ & $3.88(-2)$ \\
\hline 58.7 & & 1.19 (1) & 1.83 & $(0)$ & & \\
\hline 60 & 3.66 (1) & & & & $1.01(-1)$ & $3.18(-2)$ \\
\hline 72.9 & & $8.53(0)$ & & & & \\
\hline 73.7 & & & 1.37 & $(0)$ & & \\
\hline 75 & 2.75 (1) & & & & $7.75 \quad(-2)$ & $2.40 \quad(-2)$ \\
\hline $90 \quad \mathrm{~N}$ & & & & & $4.86(-2)$ & \\
\hline
\end{tabular}

${ }^{a}$ See experimental program plan in Appendix A for description of experiments.

$b_{\text {Read: }} 7.84 \times 10^{0}$ 
Table 26. Bonner Ball measurements on centerline at $30 \mathrm{~cm}$ behind a series of configurations (Items VA - J).

\begin{tabular}{|c|c|c|c|c|c|c|c|}
\hline \multirow[b]{2}{*}{ Conf iguration ${ }^{a}$} & \multicolumn{7}{|c|}{ Bonner Ball count rates $\left(s^{-1} w^{-1}\right)$} \\
\hline & $\begin{array}{c}\text { bare } \\
\text { detector }\end{array}$ & $\begin{array}{l}\text { CI) covered } \\
\text { detector }\end{array}$ & $\begin{array}{c}3-\text { inch } \\
\text { diam ball }\end{array}$ & $\begin{array}{l}\text { 5--inc } \\
\text { diam }\end{array}$ & $\begin{array}{l}\text { ch } \\
\text { ball l }\end{array}$ & $\begin{array}{c}\text { 8-inch } \\
\text { diam ball }\end{array}$ & $\begin{array}{c}\text { 10-inch } \\
\text { diam ball }\end{array}$ \\
\hline VA & $5.98(0)^{\mathrm{b}}$ & $2.92 \quad(0)$ & 3.44 (1) & 5.61 & (1) & $2.12(1)$ & $8.24(0)$ \\
\hline VA & $5.41(0)^{\mathrm{C}}$ & $3.02(0)^{\mathrm{C}}$ & & & & & \\
\hline VB & $1.61(0)$ & $1.11(0)$ & $1.25(1)$ & 2.03 & $(1)$ & $7.94(0)$ & $3.20 \quad(0)$ \\
\hline VB & $1.49(0)^{\mathrm{c}}$ & $1.14(0)^{c}$ & & & & & \\
\hline VC & $9.37(-1)$ & $5.23(-1)$ & $5.42(0)$ & 7.66 & $(0)$ & $2.79(0)$ & $1.09 \quad(0)$ \\
\hline VC & $8.31(-1)^{\mathrm{c}}$ & $5.42(-1)^{\mathrm{C}}$ & & & & & \\
\hline VD & $4.12(-1)$ & $2.21(-1)$ & $1.97(0)$ & 2.68 & $(0)$ & $9.37(-1)$ & $3.65(-1)$ \\
\hline VD & $3.71(-1)^{\mathrm{c}}$ & & & & & & \\
\hline VF & $1.09(-2)$ & $4.32(-3)$ & $1.99(-1)$ & 9.02 & $(-1)$ & $5.93(-1)$ & $2.98(-1)$ \\
\hline VE & $7.70(-3)^{c}$ & & & & & & \\
\hline vF & $1.33(-2)$ & $4.91(-3)$ & $1.27(-1)$ & 3.91 & $(-1)$ & $2.12(-1)$ & $9.76(-2)$ \\
\hline $\mathrm{VF}$ & $9.91(-3)^{\mathrm{C}}$ & & & & & & \\
\hline$V G$ & $8.53(-3)$ & $3.49(-3)$ & $6.33(-2)$ & 1.50 & $(-1)$ & $7.20(-2)$ & $3.13(-2)$ \\
\hline VG & $7.04(-3)^{\mathrm{c}}$ & $3.64(-3)^{\mathrm{c}}$ & & 1.55 & $(-1)$ & & \\
\hline VH & $3.77(-3)$ & $1.02(-3)$ & $5.43(-2)$ & 2.83 & $(-1)$ & $2.11(-1)$ & $1.14(-1)$ \\
\hline $\mathrm{VH}$ & $1.55(-3)^{\mathrm{C}}$ & $1.03(-3)^{c}$ & & & & & \\
\hline VI & $3.79(-3)$ & $1.18(-3)$ & $3.40(-2)$ & 1.19 & $(-1)$ & $7.18(-2)$ & $3.51(-2)$ \\
\hline VI & $2.11(-3)^{\mathrm{C}}$ & & & & & & \\
\hline VJ & $3.11(-3)$ & $9.23(-4)$ & $1.34(-2)$ & 4.94 & $(-2)$ & $2.52(-2)$ & $1.16(-2)$ \\
\hline $\mathrm{VJ}$ & 1. $74(-3)^{\mathrm{c}}$ & . & & & & & \\
\hline
\end{tabular}

\footnotetext{
a See experimental program plan in Appendix A for description of configurations.

$b_{\text {Read } 5.98 \times 10^{\circ}}$

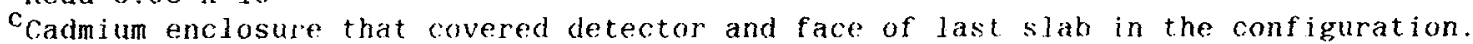


Table 27. Bonner ball measurements on centerline at 304.8 or $501.3 \mathrm{~cm}$ from the center of the reactor (Itens VA - J, VIA - F, VIIA - D)

\begin{tabular}{|c|c|c|c|c|c|c|c|c|c|c|c|c|c|c|c|}
\hline \multirow{3}{*}{ Configuration ${ }^{a}$} & \multirow{3}{*}{$\begin{array}{l}\text { Location } \\
\quad(\mathrm{cm})\end{array}$} & \multicolumn{14}{|c|}{ Bonner ball count rates $\left(\mathrm{s}^{-1} \mathrm{w}^{-1}\right)$} \\
\hline & & \multicolumn{3}{|c|}{ Bare Counter } & \multicolumn{2}{|c|}{ CD-Covered Counter } & \multicolumn{3}{|c|}{ 3-inch Diam Ball } & \multicolumn{2}{|c|}{ 5-1nch Diam Ball } & \multicolumn{2}{|c|}{ 8-inch Diam Ball } & \multicolumn{2}{|c|}{ 10-1nch Diam Ball } \\
\hline & & Foregr & round $^{b}$ & Background ${ }^{c}$ & Foreground & Background & Foreground & Backgro & ound & Foreground & Background & Foreground & Background & Foreground & Background \\
\hline $\begin{array}{l}v_{A} \\
v_{A}\end{array}$ & $\begin{array}{l}501.3 \\
501.3\end{array}$ & $\begin{array}{l}1.40 \\
8.96\end{array}$ & $\begin{array}{l}(0)^{d} \\
(-1)^{e}\end{array}$ & $4.62(-1)$ & $4.92(-1)$ & $5.78(-2)$ & $5.49(0)$ & .492 & $(-1)$ & $8.87(0)$ & $6.92(-1)$ & $3.35(0)$ & $2.45(-1)$ & $1.27(0)$ & $9.53(-2)$ \\
\hline $\begin{array}{l}\text { vB } \\
\text { vB }\end{array}$ & $\begin{array}{l}501.3 \\
501.3\end{array}$ & $\begin{array}{l}3.59 \\
2.81\end{array}$ & $(-1)$ & $1.27(-1)$ & $1.76(-1)$ & $1.78(-2)$ & $1.91(0)$ & 1.50 & $(-1)$ & $3.96(0)$ & $2.10(-1)$ & $1.18(0)$ & $7.55(-1)$ & $4.74(-1)$ & $3.19(-2)$ \\
\hline $\begin{array}{l}\mathrm{vo} \\
\mathrm{vc} \\
\mathrm{vc}\end{array}$ & $\begin{array}{l}501.3 \\
501.3\end{array}$ & $\begin{array}{l}2.89 \\
2.05\end{array}$ & $(-1)^{e}$ & $9.46(-2)$ & $1.25(-1)$ & $1.24(-2)$ & $1.22(0)$ & 1.01 & $(-1)^{\circ}$ & $1.73(0)$ & $1.19(-1)$ & $6.25(-1)$ & $3.94(-2)$ & $2.45(-1)$ & $1.56(-2)$ \\
\hline $\begin{array}{l}\text { vD } \\
\text { vD }\end{array}$ & $\begin{array}{l}501.3 \\
501.3\end{array}$ & $\begin{array}{l}1.69 \\
1.06\end{array}$ & $(-1)$ & $2.36(-2)$ & $7.46(-2)$ & $3.24(-3)$ & $6.46(-1)$ & 2.43 & $(-2)$ & $8.48(-1)$ & $3.11(-2)$ & $3.02(-1)$ & $1.04(-2)$ & $1.21(-1)$ & $4.36(-3)$ \\
\hline $\begin{array}{l}\mathrm{VE} \\
\mathrm{VE}\end{array}$ & $\begin{array}{l}501.3 \\
501.3\end{array}$ & $\begin{array}{l}8.54 \\
7.83\end{array}$ & $(-3)$ & $4.84(-3)$ & $1.77(-3)$ & $5.68(-4)$ & $4.13(-2)$ & 6.14 & $(-3)$ & $1.61|-1|$ & $1.50(-2)$ & $1.06(-1)$ & $7.49(-3)$ & $5.31(-2)$ & $3.21 \quad(-3)$ \\
\hline $\begin{array}{l}\mathrm{VF} \\
\mathrm{VF}\end{array}$ & $\begin{array}{l}501.3 \\
501.3\end{array}$ & $\begin{array}{l}7.71 \\
5.45\end{array}$ & $\left(\begin{array}{l}(-3) \\
(-3)^{\mathrm{e}}\end{array}\right.$ & $4.01(-3)$ & $1.79(-3)$ & $3.87(-4)$ & $3.51(-2)$ & 4.29 & $(-3)$ & $9.55(-2)$ & $8.42(-3)$ & $5.29(-2)$ & $3.51(-3)$ & $2.45(-2)$ & $1.66(-3)$ \\
\hline $\begin{array}{l}\text { vG } \\
\text { vG }\end{array}$ & $\begin{array}{l}501.3 \\
501.3\end{array}$ & $\begin{array}{l}5.76 \\
4.15\end{array}$ & $\begin{array}{l}(-3) \\
(-3)^{\mathrm{e}}\end{array}$ & $2.55(-3)$ & $1.55(-3)$ & $2.08(-4)$ & $2.44(-2)$ & 1.98 & $(-3)$ & $5.53(-2)$ & $3.76(-3)$ & $2.70(-2)$ & $1.52(-3)$ & $1.17(-2)$ & $6.92(-4)$ \\
\hline $\begin{array}{l}\text { vH } \\
\text { vH. }\end{array}$ & $\begin{array}{l}501.3 \\
501.3\end{array}$ & $\begin{array}{l}4.37 \\
4.08\end{array}$ & $\begin{array}{l}(-3) \\
(-3)^{e}\end{array}$ & $2.71(-3)$ & $6.21(-4)$ & $2.36(-4)$ & $1.37 \cdot(-2)$ & 2.59 & $(-3)$ & $5.64(-2)$ & $5.88(-3)$ & $4.11(-2)$ & $3.00(-3)$ & $2.24(-2)$ & $1.57(-3)$ \\
\hline $\begin{array}{l}\text { VI } \\
\text { VI }\end{array}$ & $\begin{array}{l}501.3 \\
501.3\end{array}$ & $\begin{array}{l}3.08 \\
2.48\end{array}$ & $(-3)^{\mathrm{e}}$ & $2.14(-3)$ & $5.08(-4)$ & $1.52(-4)$ & $1.02(-2)$ & 1.66 & $(-3)$ & $3.28(-2)$ & $3.47(-3)$ & $1.95(-2)$ & $1.57(-3)$ & $9.57(-3)$ & $7.21(-4)$ \\
\hline $\begin{array}{ll}\mathrm{V}^{2} \\
\mathrm{vu}\end{array}$ & 501.3 & 2.73 & $(-3)$ & $1.83(-3)$ & $4.85(-4)$ & $1.20(-4)$ & $8.01(-3)$ & 1.15 & $(-3)$ & $2.01(-2)$ & $2.19(-3)$ & $1.04(-2)$ & $9.42(-4)$ & $4.77(-3)$ & $4.41 \quad(-4)$ \\
\hline $\begin{array}{r}\text { VJ } \\
\text { VIA }\end{array}$ & $\begin{array}{l}501.3 \\
304.8\end{array}$ & $\begin{array}{l}2.22 \\
8.44\end{array}$ & $\begin{array}{l}(-3)^{\mathrm{e}} \\
(0)^{\mathrm{d}}\end{array}$ & $3: 46(0)$ & $2.62(0)$ & $3.53(-1)$ & $4.06 .(1)$ & 3.77 & (0) & & & & $2.89(0)$ & 2.22 (1) & 1.25 \\
\hline VIA & 304.8 & 5.51 & $(0)^{\mathrm{e}}$ & 0.30101 & 2.0687 & $3.001-1)$ & 9.00 .111 & 3.11 & 107 & $1.00(2)$ & $7.07(0)$ & $5.10(1)$ & 2.89101 & $2.22(1)$ & $1.25 \quad(0)$ \\
\hline $\begin{array}{l}\text { VIB } \\
\text { VIB }\end{array}$ & $\begin{array}{l}304.8 \\
304.8\end{array}$ & $\begin{array}{l}2.10 \\
5.54\end{array}$ & $\begin{array}{l}(1) \\
(0)^{\mathrm{e}}\end{array}$ & $4.06(0)$ & $2.95(0)$ & $2.33(-1)$ & 2.98 (1) & 2.00 & (0) & $5.42(1)$ & $3.17(0)$ & $2.26(1)$ & $1.14(0)$ & $9.41(0)$ & $4.88(-1)$ \\
\hline $\begin{array}{l}\text { VIC } \\
\text { VIC }\end{array}$ & $\begin{array}{l}304.8 \\
304.8\end{array}$ & $\begin{array}{l}3.11 \\
3.08\end{array}$ & (1) ${ }^{\mathrm{e}}$ & $3.17(0)$ & $1.88(0)$ & $9.00(-1)$ & 1.57 (1) & 6.65 & $(-1)$ & $2.34(1)$ & $9.55(-1)$ & $9.00(0)$ & $3.45(-1)$ & $3.64(0)$ & $1.45(-1)$ \\
\hline $\begin{array}{l}\text { VID } \\
\text { VID }\end{array}$ & $\begin{array}{l}304.8 \\
304.8\end{array}$ & $\begin{array}{l}3.33 \\
1.42\end{array}$ & $\begin{array}{l}\text { (1) } \\
(0)^{e}\end{array}$ & $2.60(0)$ & $8.80(-1)$ & $3.22(-2)$ & $6.27(0)$ & 2.20 & $(-1)$ & $8.23(0)$ & $2.87(-1)$ & $3.02(0)$ & $9.41(-2)$ & $1.21(0)$ & $3.98(-2)$ \\
\hline $\begin{array}{l}\text { VIE } \\
\text { VIE }\end{array}$ & $\begin{array}{l}304.8 \\
304.8\end{array}$ & $\begin{array}{l}3.28 \\
6.21\end{array}$ & $(1)$ & $1.80(0)$ & $3.60(-1)$ & $8.97(-3)$ & $.44(0)$ & 5.63 & $(-2)$ & $2.96(0)$ & $7.08(-2)$ & $1.02(0)$ & $2.30(-2)$ & $4.13(-1)$ & $1.10(-2)$ \\
\hline $\begin{array}{l}\text { VIF } \\
\text { VIF }\end{array}$ & $\begin{array}{l}304.8 \\
304.8\end{array}$ & $\begin{array}{l}2.98 \\
2.62\end{array}$ & $\left(\begin{array}{l}1) \\
(-1)^{e}\end{array}\right.$ & $1.10(0)$ & $1.29(-1)$ & $2.03(-3)$ & $8.05 \cdot(-1)$ & 1.28 & $(-2)$ & $9.86(-1)$ & $1.56(-2)$ & $3.23(-1)$ & $5.42(-3)$ & $1.37(-1)$ & $2.54(-3)$ \\
\hline VIIA & 304.8 & & & & & & $1.55\{1\}$ & 1.78 & 10 & $5.90(1)$ & 4.1810 & $4(1)$ & 2.4011 & 1.70 (1) & $8.98(-1)$ \\
\hline VII & & & & & & & 110 & 5.03 & $1-$ & 2.1211 & 1.2110 & $1.30 \quad(1)$ & $5.33(-1)$ & $5.89(0)$ & $2.55 \quad(-1)$ \\
\hline VIIC & 304.8 & & & & & & $1.26(-1)$ & 8.11 & $(-3)$ & $6.27 .(-1)$ & $2.04(-2)$ & $4.26(-1)$ & $9.81(-3)$ & $2.02(-1)$ & $4.39(-3)$ \\
\hline $\begin{array}{l}\text { VIID } \\
\text { VIID }\end{array}$ & 304.8 & 9.12 & $(-3)$ & $2.68(-3)$ & $3.05(-3)$ & $2.81(-4)$ & $1.04(-1)$ & 3.25 & $(-3)$. & $3.711(-1)$ & $6.98(-3)$ & $2.08(-1)$ & $2.98(-3)$ & $9.17(-2)$ & $1.40(-3)$ \\
\hline VIID & 304.8 & & 1 & & & & & & & & & & & & \\
\hline
\end{tabular}

a See experimental program plan in Appendix A for description of configurations.

Weutron $f l u x$ without shadow shield between detector and configuration.

${ }^{c}$ Neutron $f l u x$ with shadow shield between detector and configuration.

Read: $1.40 \times 10^{\circ}$

ecadmium over face of last slab in configuration.

$\mathrm{f}$ Background taken at $311.5 \mathrm{~cm}$. 
Table 28. Fast neutron fluxes $(>0.8 \mathrm{MeV})$ on centerline at $51.1 \mathrm{~cm}$ behind the graphite $(36.4 \mathrm{~cm}$ behind the lead) (Item VIA): Run 7856A.

\begin{tabular}{|c|c|c|c|c|c|c|c|c|c|c|}
\hline \multicolumn{2}{|c|}{$\begin{array}{l}\text { Neutron } \\
\text { Energy } \\
(\mathrm{MeV})\end{array}$} & \multirow{2}{*}{ Flux } & \multirow{2}{*}{$\begin{array}{c}\begin{array}{c}\text { (neutrons } \\
\text { Lower } \\
\text { Limit }\end{array} \\
3.47 \mathrm{E} \text { (3) }\end{array}$} & \multirow{2}{*}{$\mathrm{cm}^{-2}$} & \multirow{2}{*}{$\begin{array}{c}\begin{array}{c}\mathrm{MeV}^{1} \mathrm{~kW}^{-1} \mathrm{~s}^{-1} \text { ) } \\
\text { Limit }\end{array} \\
3.51 \mathrm{~F} \text { (3) }\end{array}$} & $\begin{array}{l}\text { Neutron } \\
\text { Energy } \\
(\mathrm{MeV})\end{array}$ & \multicolumn{2}{|c|}{$\begin{array}{l}\text { Flux (neutrons } \\
\text { lower } \\
\text { l.imit }\end{array}$} & \multicolumn{2}{|c|}{$\begin{array}{c}\mathrm{cm}^{-2} \mathrm{MeV}^{-1} \mathrm{~kW}^{-1} \mathrm{~s}^{-1} \text { ) } \\
\text { Upper } \\
\text { Limit }\end{array}$} \\
\hline $8.11 \mathrm{E}$ & $(-1)$ & & & & & $5.94 \mathrm{E}(0)$ & $2.44 \mathrm{E}$ & (1) & $2.58 \mathrm{E}$ & (1) \\
\hline $9.07 \mathrm{E}$ & $(-1)$ & & $3.73 \mathrm{~F},(3)$ & & $3.75 \mathrm{E}$ (3) & $6.25 E \quad(0)$ & $2.05 \mathrm{E}$ & (1) & $2.23 E$ & (1) \\
\hline $1.01 \mathrm{E}$ & (0) & & $3.28 \mathrm{E} \quad(3)$ & & $3.29 \mathrm{E} \quad(3)$ & $6.56 \mathrm{~F} \quad(0)$ & $1.74 \mathrm{E}$ & (1) & $1.90 \mathrm{E}$ & (1) \\
\hline $1.11 \mathrm{E}$ & $(0)$ & & $2.70 E \quad(3)$ & & $2.71 \mathrm{E}$ (3) & $6.84 \mathrm{E}(0)$ & $1.50 \mathrm{~F}$ & (1) & $1.60 \mathrm{E}$ & (1) \\
\hline $1.20 \mathrm{E}$ & (0) & & $2.25 E$ & & $2.26 \mathrm{E}$ (3) & $7.24 \mathrm{E} \quad(0)$ & 1. $22 \mathrm{E}$ & (1) & $1.31 \mathrm{E}$ & (1) \\
\hline $1.31 \mathrm{E}$ & $(0)$ & & $1.91 \mathrm{E}$ (3) & & $1.92 \mathrm{E}$ (3) & $7.74 \mathrm{E} \quad(0)$ & $9.32 \mathrm{E}$ & $(0)$ & $1.03 \mathrm{E}$ & (1) \\
\hline $1.41 \mathrm{E}$ & (0) & & $1.65 \mathrm{E} \quad(3)$ & & $1.66 \mathrm{E} \quad(3)$ & $8.24 E(0)$ & $6.79 \mathrm{~F}$ & $(0)$ & $7.54 \mathrm{E}$ & $(0)$ \\
\hline $1.51 E$ & $(0)$ & & $1.44 E \quad(3)$ & & $1.44 \mathrm{E} \quad(3)$ & $8.76 \mathrm{E}(0)$ & $5.63 \mathrm{E}$ & $(0)$ & $6.16 \mathrm{E}$ & (0) \\
\hline $1.61 \mathrm{E}$ & $(0)$ & & $1.23 \mathrm{E}$ (3) & & $1.23 \mathrm{E} \quad(3)$ & $9.26 \mathrm{~F},(0)$ & $5.25 \mathrm{E}$ & (0) & $5.70 \mathrm{E}$ & $(0)$ \\
\hline $1.71 \mathrm{E}$ & (0) & & 1.03E (3) & & $1.04 E \quad(3)$ & $9.74 \mathrm{E}(0)$ & $4.33 E$ & $(0)$ & $4.7 O E$ & $(0)$ \\
\hline $1.81 \mathrm{E}$ & (0) & & $8.56 \mathrm{E} \quad(2)$ & & $8.63 E$ (2) & $1.03 E(1)$ & $3.07 \mathrm{E}$ & $(0)$ & $3.34 \mathrm{E}$ & (0) \\
\hline $1.93 \mathrm{E}$ & (0) & & $6.97 \mathrm{E}$ (2) & & $7.04 \mathrm{E}$ (2) & $1,08 \mathrm{E}(1)$ & $2.29 E$ & $(0)$ & $2.49 \mathrm{E}$ & $(0)$ \\
\hline $2.10 E$ & (a) & & $5.29 E \quad$ (2) & & $5.35 E$ (2) & $1.12 \mathrm{E}$ (1) & $2.02 E$ & $(0)$ & $2.17 \mathrm{E}$ & $(0)$ \\
\hline $2.30 \mathrm{E}$ & $(0)$ & & $3.85 \mathrm{E} \quad(2)$ & & $3.90 \mathrm{E}$ (2) & $1.18 \mathrm{E}$ (1) & $1.88 \mathrm{E}$ & $(0)$ & $2.02 \mathrm{E}$ & (0) \\
\hline $2.50 \mathrm{E}$ & (0) & & $2.76 \mathrm{E} \quad(2)$ & & $2.80 \mathrm{E} \quad$ (2) & $1.24 \mathrm{E}$ (1) & $1.52 \mathrm{E}$ & $(0)$ & $1.64 \mathrm{E}$ & $(0)$ \\
\hline $2.70 \mathrm{E}$ & (0) & & $1.95 \mathrm{E}$ & & 1.99E (2) & $1.32 \mathrm{E}$ (1) & $8.02 \mathrm{E}$ & $(-1)$ & $8.79 \mathrm{E}$ & $(-1)$ \\
\hline $2.90 \mathrm{E}$ & $(0)$ & & $1.45 \mathrm{~F} \quad(2)$ & & $1.49 \mathrm{E}$ (2) & 1. 40F (1) & $2.69 \mathrm{~F}$ & $(-1)$ & $3.03 E$ & $(-1)$ \\
\hline $3.10 \mathrm{E}$ & (a) & & 1.08E (2) & & $1.12 E$ (2) & $1.48 \mathrm{E}$ (1) & $6.35 E$ & $(-2)$ & $7.73 E$ & $(-2)$ \\
\hline $3.30 \mathrm{E}$ & (0) & & $8.24 \mathrm{E}$ (1) & & $8.56 \mathrm{E}$ (1) & $1.56 \mathrm{E} \quad(1)$ & $3.62 \mathrm{E}$ & $(-3)$ & $8.88 \mathrm{E}$ & $(-3)$ \\
\hline $3.50 \mathrm{E}$ & (0) & & $6.55 \mathrm{E}$ (1) & & $6.92 \mathrm{E}(1)$ & $1.65 E$ (1) & $-5.46 \mathrm{E}$ & $(-3)$ & $-3.91 \mathrm{E}$ & $(-3)$ \\
\hline $3.71 F$ & $(0)$ & & $5.51 \mathrm{E}$ (1) & & $5.81 E \quad(1\rangle$ & $1.75 F(1)$ & $-5.09 \mathrm{E}$ & $(-3)$ & $-4.53 E$ & $(-3)$ \\
\hline $3.91 \mathrm{E}$ & $(0)$ & & $4.92 E$ (1) & & $5.18 \mathrm{E} \quad(1)$ & $1.85 E(1)$ & $-4.29 E$ & $(-3)$ & $-3.81 E$ & $(-3)$ \\
\hline $4.15 \mathrm{E}$ & (0) & & $4.75 \mathrm{E} \quad$ (1) & & $5.01 \mathrm{E}$ (1) & $1.95 \mathrm{E} \quad(1)$ & $-3.49 \mathrm{E}$ & $(-3)$ & $-3.01 \mathrm{E}$ & $(-3)$ \\
\hline $4.45 \mathrm{E}$ & (0) & & $5.03 E \quad(1)$ & & $5.25 \mathrm{E}$ (1) & $2.06 \mathrm{E}(1)$ & $-2.53 E$ & $(-3)$ & $-2.06 \mathrm{E}$ & $(-3)$ \\
\hline $4.75 \mathrm{E}$ & $(0)$ & & $4.96 \mathrm{~F} \quad$ (1) & & $5.16 \mathrm{E}$ (1) & 2. 16F (1) & $-1.60 \mathrm{E}$ & $(-3)$ & $-1.14 \mathrm{E}$ & $(-3)$ \\
\hline $5.04 E$ & $(0)$ & & $4.24 E(1)$ & & $4.41 E$ (1) & $2.26 E(1)$ & $-9.71 E$ & $(-4)$ & $-5.14 \mathrm{E}$ & $(-4)$ \\
\hline $5.35 \mathrm{E}$ & (0) & & $3.41 \mathrm{E}(1)$ & & $3.57 \mathrm{~F} \quad(1)$ & $2.35 E$ (1) & $-5.87 \mathrm{E}$ & $(-4)$ & $-1.01 \mathbf{E}$ & $(-4)$ \\
\hline $5.65 \mathrm{E}$ & $(0)$ & & $2.85 \mathrm{E}(1)$ & & $3.01 E(1)$ & & & & & \\
\hline
\end{tabular}

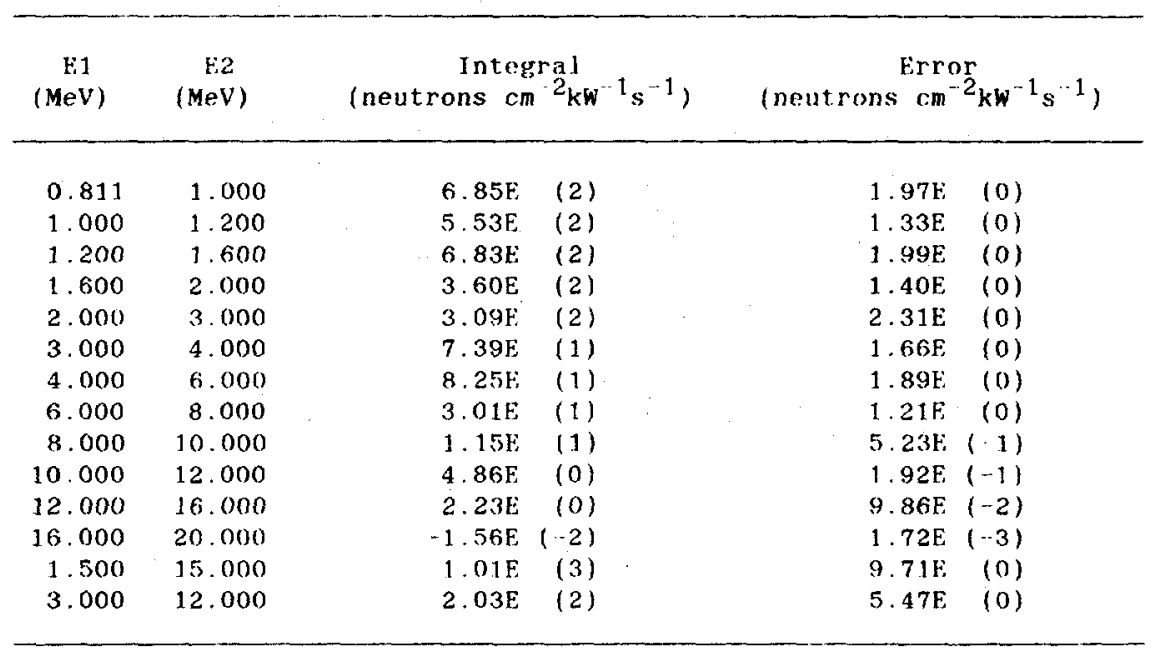


Table 29. Neutron fluxes ( $50 \mathrm{keV}$ to $1.4 \mathrm{MeV}$ ) on centerline at $51.1 \mathrm{~cm}$ behind the graphite $(36.4 \mathrm{~cm}$ behind the lead)

(Iten VIA): Runs 1542A, 1541B, 1541A.

\begin{tabular}{|c|c|c|c|c|}
\hline $\mathrm{N}$ & \multicolumn{2}{|c|}{$\begin{array}{c}\text { Energy Boundary } \\
(\mathrm{MeV})\end{array}$} & (Neutron $\mathrm{cm}^{-\mathrm{Flux}} \mathrm{MeV}^{-1} \mathrm{~kW}^{-1} \mathrm{~s}^{-1}$ ) & \multirow[t]{2}{*}{$\begin{array}{l}\text { Error } \\
(\%)\end{array}$} \\
\hline \multicolumn{4}{|r|}{ Run 1542A } & \\
\hline 1 & 0.0542 & 0.0633 & $2.77 \mathrm{E} \quad(5)$ & 1.32 \\
\hline 2 & 0.0633 & 0.0762 & $1.92 \mathrm{E} \quad(5)$ & 1.42 \\
\hline 3 & 0.0762 & 0.0890 & $1.64 \mathrm{E}$ (5) & 1.82 \\
\hline 4 & 0.0890 & 0.1037 & $1.35 \mathrm{E} \quad(5)$ & 2.06 \\
\hline 5 & 0.1037 & 0.1221 & $1.14 \mathrm{E}(5)$ & 2.03 \\
\hline 6 & 0.1221 & 0.1441 & $9.98 \mathrm{E} \quad$ (4) & 2.03 \\
\hline 7 & 0.1441 & 0.1698 & $7.69 \mathrm{E} \quad(4)$ & 2.39 \\
\hline 8 & 0.1698 & 0.1992 & $6.51 \mathrm{E}(4)$ & 2.61 \\
\hline 9 & 0.1992 & 0.2359 & $5.45 \mathrm{E} \quad(4)$ & 2.52 \\
\hline \multicolumn{5}{|c|}{ Run $1541 \mathrm{~B}$} \\
\hline 1 & 0.1672 & 0.1993 & $6.13 E \quad(4)$ & 0.85 \\
\hline 2 & 0.1993 & 0.2359 & $5.22 \mathrm{E} \quad(4)$ & 0.93 \\
\hline 3 & 0.2359 & 0.2771 & $4.19 \mathrm{E} \quad(4)$ & 1.10 \\
\hline 4 & 0.2771 & 0.3230 & $3.46 \mathrm{E} \quad(4)$ & 1.27 \\
\hline 5 & 0.3230 & 0.3825 & $2.70 \mathrm{E} \quad(4)$ & 1.26 \\
\hline 6 & 0.3825 & 0.4466 & $2.04 \mathrm{E} \quad(4)$ & 1.64 \\
\hline 7 & 0.4466 & 0.5291 & $1.73 E \quad(4)$ & 1.48 \\
\hline \multicolumn{5}{|c|}{ Run 1541A } \\
\hline 1 & 0.3793 & 0.4448 & $2.14 \mathrm{E} \quad(4)$ & 0.82 \\
\hline 2 & 0.4448 & 0.5291 & $1.78 \mathrm{E} \quad(4)$ & 0.77 \\
\hline 3 & 0.5291 & 0.6227 & $1.30 \mathrm{E} \quad(4)$ & 0.95 \\
\hline 4 & 0.6227 & 0.7258 & $9.39 \mathrm{E} \quad(3)$ & 1.21 \\
\hline 5 & 0.7258 & 0.8569 & $5.83 \mathrm{E} \quad(3)$ & 1.49 \\
\hline 6 & 0.8569 & 1.0067 & $4.16 \mathrm{E}$ & 1.86 \\
\hline 7 & 1.0067 & 1.1846 & $3.07 \mathrm{E}(3)$ & 2.09 \\
\hline 8 & 1.1846 & 1.4000 & $2.17 \mathrm{E} \quad(3)$ & 2.33 \\
\hline
\end{tabular}


Table 30. 5-inch Bonner ball horizontal traverses through midplane at $30 \mathrm{~cm}$ behind a series of configurations (Items VIA, B, D, F, VIIA - C).

\begin{tabular}{|c|c|c|c|c|c|c|c|c|c|c|c|}
\hline \multirow{2}{*}{$\begin{array}{l}\text { Distance from } \\
\text { centerline } \\
(\mathrm{cm})\end{array}$} & \multicolumn{11}{|c|}{ Bonner Bald count rates $\left(s^{-1} W^{-1}\right)$} \\
\hline & Item VIA & Item VIB ${ }^{a}$ & Item $v$ & VID ${ }^{a}$ & Item & VIF ${ }^{a}$ & Item VIIA ${ }^{a}$ & Item & $\operatorname{VIIB} \mathrm{a}^{\mathrm{a}}$ & Item $v$ & $\operatorname{VII} \mathrm{C}^{\mathrm{a}}$ \\
\hline $76.2 S$ & $1.78(2)^{\mathrm{b}}$ & $7.99 \quad(1)$ & 9.60 & $(0)$ & 7.63 & $(-1)$ & $9.63(1)$ & 2.58 & (1) & 6.24 & $(-1)$ \\
\hline 60 & 2.79 (2) & $1.26 \quad(2)$ & 1.55 & (1) & 1.24 & (0) & 1.43 (2) & 4.01 & (1) & 9.62 & $(-1)$ \\
\hline 45 & $3.80 \quad(2)$ & 1.69 (2) & 2.13 & (1) & 1.71 & (0) & 1.89 (2) & 5.39 & (1) & 1.27 & (0) \\
\hline 30 & $4.55 \quad(2)$ & $2.08 \quad(2)$ & 2.66 & (1) & 2.11 & (0) & 2.26 (2) & 6.52 & (1) & 1.50 & (0) \\
\hline 15 & 5.12 (2) & $2.34 \quad(2)$ & 2.99 & $(1)$ & 2.37 & (0) & 2.51 (2) & 7.24 & (1) & 1.64 & (0) \\
\hline 0 & $5.38 \quad(2)$ & 2.43 (2) & 3.10 & $(1)$ & 2.45 & (0) & 2.64 (2) & 7.53 & (1) & 1.65 & (0) \\
\hline 15 & 5.06 (2) & 2.36 (2) & 2.98 & (1) & 2.34 & (0) & $2.51 \quad(2)$ & 7.05 & (1) & 1.57 & (0) \\
\hline 30 & 4.50 (2) & $2.08 \quad(2)$ & 2.63 & (1) & 2.05 & (0) & $2.19 \quad(2)$ & 6.28 & (1) & 1.39 & (0) \\
\hline 45 & 3.53 (2) & 1.67 (2) & 2.12 & $(1)$ & 1,64 & (0) & 1.79 (2) & 5.09 & (1) & 1.14 & (0) \\
\hline 60 & 2.58 (2) & 1.23 (2) & 1.53 & (1) & 1.16 & (0) & $1.32 \quad(2)$ & 3.74 & (1) & 8.44 & $(\cdots 1)$ \\
\hline $76.2 \mathrm{~N}$ & 1.62 (2) & 7.93 (1) & 9.48 & $(0)$ & 7.31 & $(-1)$ & $8.90 \quad(1)$ & 2.36 & (1) & 5.62 & $(-1)$ \\
\hline
\end{tabular}

a See experimental program plan in Appendix A for description of experiments.

Read: $1.78 \times 10^{2}$. 
Table 31. Bonner Ball measurements on centerline at $30 \mathrm{~cm}$ behind a series of configurations (Itens VIA - F, VIIA - D).

\begin{tabular}{|c|c|c|c|c|c|c|c|c|c|c|c|c|}
\hline \multirow{3}{*}{ Configuration ${ }_{\text {VIA }}^{a}$} & \multicolumn{12}{|c|}{ Bonner Ball count } \\
\hline & \multicolumn{2}{|c|}{$\begin{array}{c}\text { bare } \\
\text { detector }\end{array}$} & \multicolumn{2}{|c|}{$\begin{array}{l}\mathrm{CD} \text {-covered } \\
\text { detector }\end{array}$} & \multicolumn{2}{|c|}{$\begin{array}{c}3 \text {-inch } \\
\text { diam ball }\end{array}$} & \multicolumn{2}{|c|}{$\begin{array}{c}\text { 5-inch } \\
\text { diam ball }\end{array}$} & \multicolumn{2}{|c|}{$\begin{array}{c}\text { 8-inch } \\
\text { diam ball }\end{array}$} & \multicolumn{2}{|c|}{$\begin{array}{c}\text { 10 inch } \\
\text { diam ball }\end{array}$} \\
\hline & 2.87 & $(1)^{b}$ & 1.25 & (1) & 2.01 & (2) & 5.15 & (2) & 2.61 & (2) & 1.10 & (2) \\
\hline VIA & 2.48 & $(1)^{\circ}$ & & & & & & & & & & \\
\hline VIB & 8.40 & (1) & 1.30 & (1) & 1.35 & (2) & 2.46 & (2) & 1.03 & (2) & 4.25 & (1) \\
\hline VIB & 7.65 & $(1)^{c}$ & & & & & & & & & & \\
\hline VIC & 1.19 & (2) & 7.42 & $(0)$ & 6.30 & (1) & 9.45 & (1) & 3.62 & (1) & 1.47 & (1) \\
\hline VIC & 1.08 & $(2)^{\mathrm{C}}$ & & & & & & & & & & \\
\hline VID & 1.13 & (2) & 3.09 & $(0)$ & 2.23 & (1) & 2.97 & (1) & 1.06 & (1) & 4.32 & $(0)$ \\
\hline VID & 1.05 & $(2)^{c}$ & & & & & & & & & & \\
\hline VIE & 9.70 & (1) & 1.08 & $(0)$ & 7.44 & $(0)$ & 9.04 & (0) & 3.08 & $(0)$ & 1.25 & (0) \\
\hline VIE & 9.19 & $(1)^{c}$ & & & & & & & & & & \\
\hline VIF & 7.52 & (1) & 3.39 & $(-1)$ & 2.10 & $(0)$ & 2.42 & (0) & 8.44 & $(-1)$ & 3.58 & $(-1)$ \\
\hline VIF & 7.49 & $(1)^{\mathrm{c}}$ & & & & & & & & & & \\
\hline VIIA & & & & & 6.57 & (1) & 2.61 & (2) & 1.69 & (2) & 7.77 & (1) \\
\hline VIIB & & & & & 1.92 & (1) & 7.69 & (1) & 4.64 & (1) & 2.13 & (1) \\
\hline VIIC & & & & & 3.04 & $(-1)$ & 1.61 & (0) & 1.08 & (0) & 5.03 & $(-1)$ \\
\hline VIID & 1.01 & $(-2)$ & 4.00 & $(-3)$ & 1.52 & $(-1)$ & 5.46 & $(-1)$ & 3.11 & $(-1)$ & 1. 37 & $(-1)$ \\
\hline VIII) & 6.39 & $(-3)^{c}$ & & & & & & & & & & \\
\hline
\end{tabular}

a See experimental program plan on Appendix A for description of configurations.

Bead: $2.87 \times 10^{1}$.

${ }^{c}$ Cadmium enclosure that surrounded the detector except on the side of the last slab in the configuration. 
Table 32. Fast Neutron fluxes $(>0.8 \mathrm{MeV})$ on centerline at $63.2 \mathrm{~cm}$ beyond the graphite in the configuration (Item VIF): Run 7859A

\begin{tabular}{|c|c|c|c|c|c|c|c|c|c|}
\hline \multicolumn{2}{|c|}{$\begin{array}{l}\text { Neutron } \\
\text { Energy } \\
(\mathrm{MeV})\end{array}$} & \multirow{2}{*}{ Flux } & \multicolumn{2}{|c|}{$\begin{array}{l}\text { (neutrons } \\
\text { Lower } \\
\text { Limit }\end{array}$} & \multicolumn{2}{|c|}{$\begin{array}{c}\left.\mathrm{cm}^{-2} \mathrm{MeV}^{-1} \mathrm{~kW}^{-1} \mathrm{~s}^{-1}\right) \\
\text { Upper } \\
\text { I.imit }\end{array}$} & $\begin{array}{l}\text { Neutron } \\
\text { Energy } \\
(\mathrm{MeV})\end{array}$ & $\begin{array}{l}\text { Flux Ineutrons } \\
\text { Lower } \\
\text { Limit }\end{array}$ & 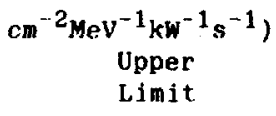 \\
\hline $8.11 E$ & $(-1)$ & & $4.48 E$ & $(0)$ & $4.54 E$ & (0) & $5.94 E \quad(0)$ & $1.06 \mathrm{E}(-1)$ & $1.10 E(-1)$ \\
\hline $9.07 \mathrm{E}$ & $(-1)$ & & $4.93 \mathrm{E}$ & $(0)$ & $4.97 \mathrm{E}$ & $(0)$ & $6.25 \mathrm{E}(0)$ & $1.00 \mathrm{E}(-1)$ & $1.06 \mathrm{E}(-1)$ \\
\hline $1.01 \mathrm{~F}$ & $(0)$ & & $4.36 \mathrm{E}$ & $(0)$ & $4.38 \mathrm{~F}$ & $(0)$ & $6.56 \mathrm{E} \quad(0)$ & $9.36 \mathrm{E} \quad(-2)$ & $9.81 E(-2)$ \\
\hline $1.11 \mathrm{E}$ & $(0)$ & & $3.54 E$ & $(0)$ & $3.57 \mathrm{E}$ & $(0)$ & $6.84 \mathrm{E}(0)$ & $8.23 E \quad(-2)$ & 8.53E $(-2)$ \\
\hline $1.20 \mathrm{~F}$ & $(0)$ & & $2.97 \mathrm{E}$ & $(0)$ & $2.99 \mathrm{E}$ & (0) & $7.24 \mathrm{E}(0)$ & $6.04 \mathrm{E}(-2)$ & $6.27 E(-2)$ \\
\hline $1.31 E$ & $(0)$ & & $2.61 \mathrm{E}$ & $(0)$ & $2.63 E$ & (0) & $7.74 \mathrm{E} \quad(0)$ & $3.59 \mathrm{E} \quad(-2)$ & $3.83 E(-2)$ \\
\hline $1.41 \mathrm{E}$ & $(0)$ & & $2.38 \mathrm{E}$ & $(0)$ & $2.40 \mathrm{~F}$ & (0) & 8. $24 \mathrm{E}(0)$ & $2.15 F(-2)$ & 2. 33E $(-2)$ \\
\hline $1.51 \mathrm{E}$ & $(0)$ & & $2.16 \mathrm{E}$ & $(0)$ & $2.18 \mathrm{E}$ & $(0)$ & $8.76 \mathrm{E}(0)$ & $1,45 \mathrm{E}(-2)$ & 1. $58 \mathrm{E}(-2)$ \\
\hline $1.61 \mathrm{~F}$ & (0) & & $1.93 \mathrm{E}$ & $(0)$ & $1.95 \mathrm{E}$ & $(0)$ & $9.26 \mathrm{E}(0)$ & $1.13 \mathrm{E}(-2)$ & $1.24 \mathrm{E}(-2)$ \\
\hline $1.71 \mathrm{E}$ & $(0)$ & & $1.69 \mathrm{E}$ & $(0)$ & $1.70 \mathrm{E}$ & $(0)$ & $9.74 \mathrm{E}(0)$ & $8.35 \mathrm{E}(-3)$ & $9.30 \mathrm{E}(-3)$ \\
\hline $1.81 E$ & $(0)$ & & $1.45 \mathrm{E}$ & $(0)$ & $1.47 \mathrm{E}$ & (0) & 1.03E (1) & $5.17 \mathrm{~F},(-3)$ & $5.94 E(-3)$ \\
\hline $1.93 \mathrm{E}$ & $(0)$ & & $1.22 \mathrm{E}$ & $(0)$, & $1.23 E$ & (0) & $1.08 \mathrm{E}$ (1) & $3.21 \mathrm{E} \quad(-3)$ & $3.87 E(-3)$ \\
\hline $2.10 \mathrm{E}$ & $(0)$ & & $9.49 E$ & $(-1)$ & $9.61 \mathrm{E}$ & $(-1)$ & $1.12 E(1)$ & $2.53 E(-3)$ & $3.11 E(-3)$ \\
\hline $2.30 \mathrm{E}$ & (0) & & $6.86 \mathrm{E}$ & $(-1)$ & $6.97 \mathrm{E}$ & $(-1)$ & $1.18 \mathrm{E} \quad(1)$ & $2.13 E(-3)$ & $2.66 E(-3)$ \\
\hline $2.50 \mathrm{E}$ & $(0)$ & & $4.87 E$ & $(-1)$ & $4.96 \mathrm{E}$ & $(-1)$ & $1.24 \mathrm{E}$ (1) & $1.48 \mathrm{E}(-3)$ & $1.91 \mathrm{E}(-3)$ \\
\hline $2.70 E$ & (0) & & $3.53 \mathrm{E}$ & $(-1)$ & $3.62 \mathrm{E}$ & $(-1)$ & $1.32 \mathrm{E}$ (1) & $7.20 \mathrm{E} \quad(-4)$ & $1.07 \mathrm{E}(-3)$ \\
\hline $2.90 \mathrm{E}$ & (0) & & $2.70 \mathrm{E}$ & $(-1)$ & $2.79 \mathrm{E}$ & $(-1)$ & $1.40 \mathrm{E}(1)$ & $4.31 \mathrm{E}(-4)$ & $7.24 \mathrm{E}(-4)$ \\
\hline $3.10 E$ & $(0)$ & & $2.09 E$ & $(-1)$ & $2.19 E$ & $(-1)$ & $1.48 E(1)$ & $3.31 E(-4)$ & $7.40 E(-4)$ \\
\hline $3.30 \mathrm{E}$ & $(0)$ & & $1.66 \mathrm{E}$ & $(-1)$ & $1.74 \mathrm{E}$ & $(-1)$ & $1.56 \mathrm{E} \quad(1)$ & $2.70 \mathrm{E}(-4)$ & $7.29 \mathrm{E} \quad(-4)$ \\
\hline $3.50 \mathrm{E}$ & $(0)$ & & $1.46 \mathrm{E}$ & $(-1)$ & $1.55 \mathrm{E}$ & $(-1)$ & $1.65 \mathrm{E}$ (1) & 1.52E $(-4)$ & $4.96 E(-4)$ \\
\hline $3.71 \mathrm{E}$ & $(0)$ & & $1.41 \mathrm{~F}$ & $(-1)$ & $1,48 \mathrm{E}$ & $(-1)$ & $1.75 \mathrm{E}$ (1) & $-8.63 E(-5)$ & $3.49 \mathrm{E} \quad(-4)$ \\
\hline $3.91 \mathrm{E}$ & $(0)$ & & $1.36 \mathrm{E}$ & $(-1)$ & $1.42 \mathrm{E}$ & $(-1)$ & $1.85 \mathrm{E}(1)$ & $-2.33 E(-4)$ & $2.52 E(-4)$ \\
\hline $4.15 \mathrm{E}$ & $(0)$ & & $1.30 \mathrm{E}$ & $(-1)$ & $1.37 \mathrm{E}$ & $(-1)$ & $1.95 \mathrm{E}(1)$ & $-2.31 \mathrm{E}(-4)$ & $2.21 \mathrm{E}(-4)$ \\
\hline $4.45 E$ & $(0)$ & & $1.32 \mathrm{E}$ & $(-1)$ & $1.38 \mathrm{E}$ & $(-1)$ & $2.06 E$ (1) & $-2.53 E(-4)$ & $2.05 E(-4)$ \\
\hline $4.75 E$ & $(0)$ & & $1.28 E$ & $(-1)$ & $1.34 E$ & $(-1)$ & $2.16 E(1)$ & $-1.82 E(-4)$ & $2.64 E(-4)$ \\
\hline $5.04 E$ & $(0)$ & & $1.17 \mathrm{E}$ & $(-1)$ & $1.22 \mathrm{E}$ & $(-1)$ & $2.26 \mathrm{E}(1)$ & $-1.91 E(-4)$ & $2.63 E(-4)$ \\
\hline $5.35 \mathrm{E}$ & $(0)$ & & $1.11 \mathrm{~F}$ & $(-1)$ & $1.15 \mathrm{E}$ & $(-1)$ & $2.35 \mathrm{E}$ (1) & $-2.00 \mathrm{E} \quad(-4)$ & $2.93 E(-4)$ \\
\hline $5.65 \mathrm{E}$ & $(0)$ & & $1.09 E$ & $(-1)$ & 1. $14 \mathrm{E}^{\prime}$ & $(-1)$ & & & \\
\hline
\end{tabular}

\begin{tabular}{rrrr}
$\begin{array}{c}\text { N1 } \\
(\mathrm{MeV})\end{array}$ & $\begin{array}{c}\mathrm{E} 2 \\
(\mathrm{MeV})\end{array}$ & $\begin{array}{c}\text { Integral } \\
\left.\text { (neutrons } \mathrm{cm}^{-2} \mathrm{~kW}^{-1} \mathrm{~s}^{-1}\right)\end{array}$ & $\begin{array}{c}\text { Error } \\
\left.\text { (neutrons } \mathrm{cm}^{-2} \mathrm{~kW}^{-1} \mathrm{~s}^{-1}\right)\end{array}$ \\
\hline 0.811 & 1.000 & $9.04 \mathrm{E}(-1)$ & $3.44 \mathrm{E}(-3)$ \\
1.000 & 1.200 & $7.31 \mathrm{E}(-1)$ & $2.45 \mathrm{E}(-3)$ \\
1.200 & 1.600 & $9.73 \mathrm{E}(-1)$ & $3.81 \mathrm{E}(-3)$ \\
1.600 & 2.000 & $6.02 \mathrm{E}(-1)$ & $2.81 \mathrm{E}(-3)$ \\
2.000 & 3.000 & $5.55 \mathrm{E}(-1)$ & $5.05 \mathrm{E}(-3)$ \\
3.000 & 4.000 & $1.64 \mathrm{E}(-1)$ & $4.08 \mathrm{E}(-3)$ \\
4.000 & 6.000 & $2.45 \mathrm{E}(-1)$ & $5.26 \mathrm{E}(-3)$ \\
6.000 & 8.000 & $1.44 \mathrm{E}(-1)$ & $3.34 \mathrm{E}(-3)$ \\
8.000 & 10.000 & $2.93 \mathrm{E}(-2)$ & $1.29 \mathrm{E}(-3)$ \\
10.000 & 12.000 & $7.25 \mathrm{E}(-3)$ & $6.39 \mathrm{E}(-4)$ \\
12.000 & 16.000 & $3.37 \mathrm{E}(-3)$ & $7.78 \mathrm{E}(-4)$ \\
16.000 & 20.000 & $4.74 \mathrm{E}(-4)$ & $2.68 \mathrm{E}(-4)$ \\
1.500 & 15.000 & $1.96 \mathrm{E}(0)$ & $1.46 \mathrm{E}(-2)$ \\
3.000 & 12.000 & $5.89 \mathrm{E}(-1)$ & \\
\hline
\end{tabular}


Table 33. Neutron fluxes (50 keV to $1.4 \mathrm{MeV}$ ) on centerline at $63.2 \mathrm{~cm}$ beyond the graphite in the configuration (Item VIF): Runs 1544A, 1544B, 1545A.

\begin{tabular}{|c|c|c|c|c|}
\hline $\mathrm{N}$ & Energy & $\begin{array}{l}\text { oundary } \\
\mathrm{eV})\end{array}$ & 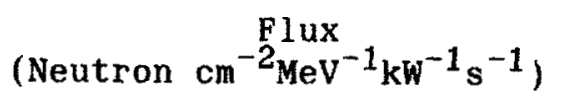 & $\begin{array}{c}\text { Error } \\
(\%)\end{array}$ \\
\hline \multicolumn{5}{|c|}{ Run 1544A } \\
\hline 1 & 0.0536 & 0.0630 & $3.62 \mathrm{E}$ (1) & 7.65 \\
\hline 2 & 0.0630 & 0.0743 & $3.43 \mathrm{E} \quad(1)$ & 7.46 \\
\hline 3 & 0.0743 & 0.0875 & $3.28 \mathrm{E}$ (1) & 7.37 \\
\hline 4 & 0.0875 & 0.1026 & $2.52 E(1)$ & 9.25 \\
\hline 5 & 0.1026 & 0.1214 & $1.94 \mathrm{E}(1)$ & 10.55 \\
\hline 6 & 0.1214 & 0.1421 & $1.84 \mathrm{E}$ (1) & 11.39 \\
\hline 7 & 0.1421 & 0.1666 & $1.62 E(1)$ & 12.06 \\
\hline 8 & 0.1666 & 0.1967 & $1.53 \mathrm{E}$ (1) & 11.27 \\
\hline 9 & 0.1967 & 0.2324 & $1.32 \mathrm{E}(1)$ & 11.96 \\
\hline \multicolumn{5}{|c|}{ Run 1544B } \\
\hline 1 & 0.1696 & 0.1989 & $1.37 E \quad(1)$ & 5.47 \\
\hline 2 & 0.1989 & 0.2324 & $1.34 \mathrm{E}$ (1) & 5.45 \\
\hline 3 & 0.2324 & 0.2743 & $1.17 \mathrm{E}$ (1) & 5.50 \\
\hline 4 & 0.2743 & 0.3204 & $1.09 E(1)$ & 6.06 \\
\hline 5 & 0.3204 & 0.3790 & $9.24 \mathrm{E} \quad(0)$ & 6.06 \\
\hline 6 & 0.3790 & 0.4460 & $7.88 \mathrm{E}(0)$ & 6.94 \\
\hline 7 & 0.4460 & 0.5256 & $8.69 \mathrm{E}(0)$ & 5.72 \\
\hline \multicolumn{5}{|c|}{ Run 1545A } \\
\hline 1 & 0.3767 & 0.4512 & $7.81 \mathrm{E} \quad(0)$ & 2.20 \\
\hline 2 & 0.4512 & 0.5256 & $8.32 E(0)$ & 2.34 \\
\hline 3 & 0.5256 & 0.6186 & $7.31 E(0)$ & 2.26 \\
\hline 4 & 0.6186 & 0.7302 & $6.66 \mathrm{E} \quad(0)$ & 2.19 \\
\hline 5 & 0.7302 & 0.8605 & $5.48 \mathrm{E}(0)$ & 2.40 \\
\hline 6 & 0.8605 & 1.0093 & $4.43 E \quad(0)$ & 2.70 \\
\hline 7 & 1.0093 & 1.1860 & $3.33 E \quad(0)$ & 3.04 \\
\hline 8 & 1.1860 & 1.4000 & $2.36 \mathrm{E}(0)$ & 3.50 \\
\hline
\end{tabular}




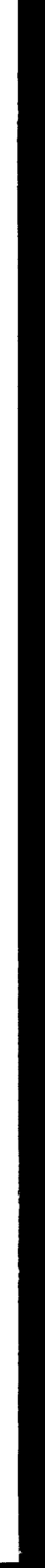




\section{APPENDIX C}

\section{List of Pigures}

Figure 1. Schematic of SM1 (Fe + Al + boral + radial blanket) (Item IA). Note: Lithiated paraffin covers lateral sides of configuration

Figure 2. Schematic of radial blanket slab containing $\mathrm{UO}_{2}$

Figure 3. Schematic of iron, aluminum, boral and sodium spectrum modifier (SM2) (Item VA). Note: Lithiated paraffin covers lateral sides of configuration

Figure 4. Schematic of stainless steel containers used for boron carbide shield slabs

Figure 5. Spectrum of high-energy neutrons $(>0.8 \mathrm{MeV})$ on centerline 178.8 $\mathrm{cm}$ beyond SM1 (Item IA): Run 7856A

Figure 6. Neutron spectrum (50 keV to $1.4 \mathrm{MeV})$ on centerline $178.8 \mathrm{~cm}$ beyond SM1 (Item IA): Runs 1537C, 1537A, 1536A

Figure 7. Schematic of SM1 plus shield configurations for Items IIA, B, $C, D, E$. Note: Lithiated paraffin covers lateral sides of configuration

Figure 8, Schematic of SM1 plus shield configurations for Item IID1, 2. Note: Lithiated paraffin covers lateral sides of configuration

Figure 9. Spectrum of high-energy neutrons (>0.8 MeV) on centerline 47.3 $\mathrm{cm}$ beyond $\mathrm{B}_{4} \mathrm{C}(33.6 \mathrm{~cm}$ beyond $\mathrm{Pb})$ (Item IID): Run $7857 \mathrm{~A}$

Figure 10. Neutron spectrum (50 keV to $1.4 \mathrm{MeV})$ on centerline $47.3 \mathrm{~cm}$ beyond $\mathrm{B}_{4} \mathrm{C}(33.6 \mathrm{~cm}$ beyond $\mathrm{Pb})$ (Item IID): Runs 1540A, 1539B, 1539A

Figure 11. Schematic of SM1 plus shield configurations for Items IIIA, C, $D$, E. Note: Lithiated paraffin covers lateral sides of configuration

Figure 12. Schematic of SM1 plus shield configuration for Item IIIB. Note: Lithiated paraffin covers lateral sides of configuration

Figure 13. Schematic of SM1 plus shield configurations for Item IVA. Note: Lithiated paraffin covers lateral sides of configuration

Figure 14. Schematic of SM1 plus shield configuration for Item IVB. Note: Lithiated paraffin covers lateral sides of configuration

Figure 15. Schematic of SM1 plus shield configuration Item IVC for spectral measurements. Note: Lithiated paraffin covers lateral sides of configuration

Figure 16. Spectrum of high-energy neutrons $(>0.8 \mathrm{MeV})$ on centerline 42.8 $\mathrm{cm}$ beyond $\mathrm{SS}(32.9 \mathrm{~cm}$ beyond $\mathrm{Pb})$ (Item IVC): Run 7860A

Figure 17. Neutron spectrum $(50 \mathrm{keV}$ to $1.4 \mathrm{MeV})$ on centerline $42.8 \mathrm{~cm}$ beyond SS $(32.9 \mathrm{~cm}$ beyond $\mathrm{Pb})$ (Item IVC): Runs 1546A, 1546B, $1547 \mathrm{~A}$ 
Figure 18. Schematic of SM1 plus shield configurations for Items IVC-I. Note: Lithiated paraffin covers lateral sides of configuration

Figure 19. Schematic of SM1 plus shield configuration for Item IVIA. Note: Lithiated paraffin covers lateral sides of configuration

Figure 20. Schematic of SM1 plus shield configuration for Item IVJ. Note: Lithiated paraffin covers lateral sides of configuration

Figure 21. Schematic of SM1 plus shield configuration for IVK. Note: Lithiated paraffin covers lateral sides of configuration

Figure 22. Schematic of SM1 plus shield configuration for Item IVL. Note: Lithiated paraffin covers lateral sides of configuration

Figure 23. Schematic of SM1 plus shield configuration for Item IVM. Note: Lithiated paraffin covers lateral sides of configuration

Figure 24. Schematic of SM2 plus shield configurations for Items VB, C, D. Note: Lithiated parafin covers lateral sides of configurations

Figure 25. Schematic of SM2 plus shield configurations for Items VE, F, $G$. Note: Lithiated parafin covers lateral sides of configurations

Figure 26. Schematic of SM2 plus shield configurations for Items VH, I, J. Note: Lithiated parafin covers lateral sides of configurations

Figure 27 Schematic of SM1 plus shield configuration for Item VIA. Note: Lithiated parafin covers lateral sides of configuration

Figure 28. Fast neutron fluxes $(>0.8 \mathrm{MeV})$ on centerline at $51.1 \mathrm{~cm}$ beyond the graphite $(36.4 \mathrm{~cm}$ behind the lead) (Item VIA): Run 7858A

Figure 29. Neutron fluxes $(50 \mathrm{keV}$ to $1.4 \mathrm{MeV})$ on centerline at $51.1 \mathrm{~cm}$ beyond the graphite $(36.4 \mathrm{~cm}$ behind the lead) (Item VIA): Runs $1542 \mathrm{~A}, 1541 \mathrm{~B}, 1541 \mathrm{~A}$

Figure 30. Schematic of SM1 plus shield configurations for Items VIA - F. Note: Lithiated paraffin covers lateral sides of configurations

Figure 31. Schematic of SM1 plus shield configuration for Item VIF. Note: Lithiated paraffin covers lateral sides of configuration

Figure 32. Spectrum of high-energy neutrons $(>0.8 \mathrm{MeV})$ on centerline 63.2 $\mathrm{cm}$ beyond the graphite in the configuration (Item VIF): Run $7859 \mathrm{~A}$

Figure 33. Neutron spectrum (50 keV to $1.4 \mathrm{MeV}$ ) on centerline $63.2 \mathrm{~cm}$ beyond the graphite in the configuration (Item VIF): Runs $1544 \mathrm{~A}, 1544 \mathrm{~B}, 1545 \mathrm{~A}$

Figure 34. Schematic of SM1 plus shield configurations for Items VIIA-D. Note: Lithiated paraffin covers lateral sides of configurations 
0
0
0
0
0
0
0
$\vdots$
0
$\vdots$
$\vdots$

0

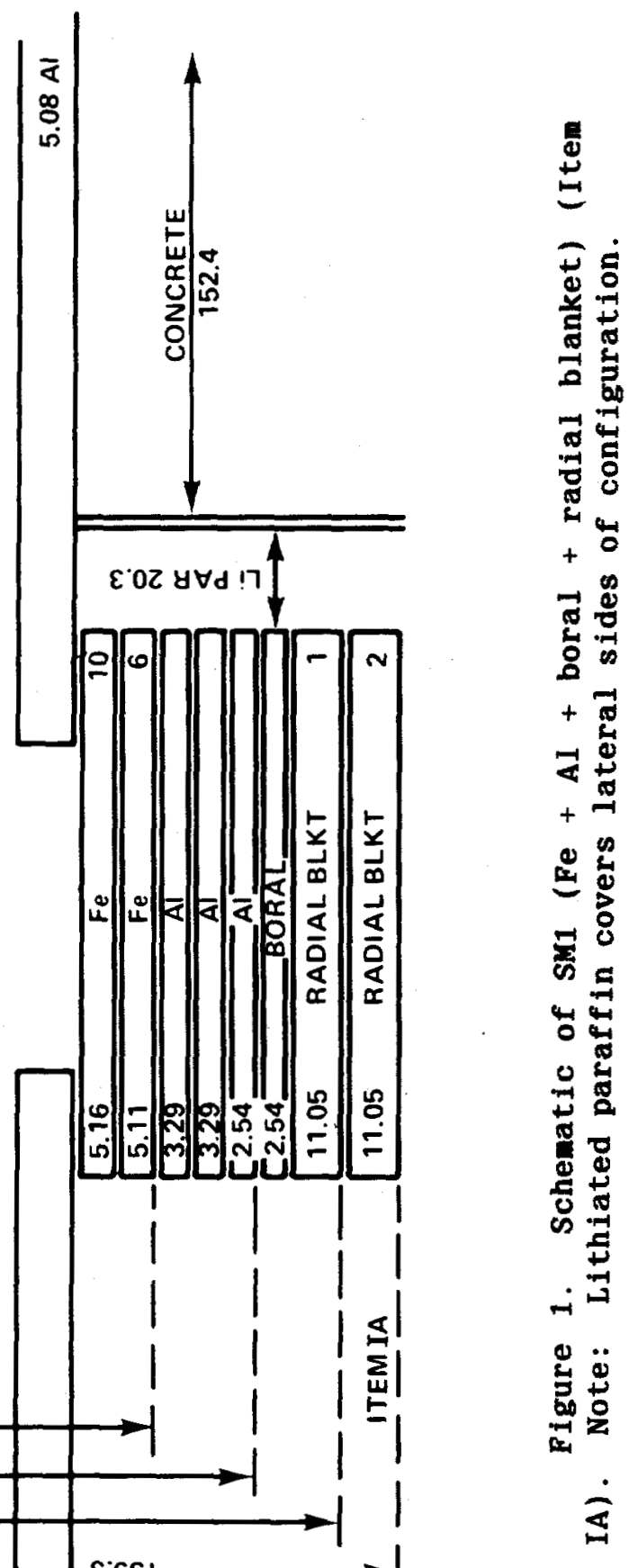


RADIAL BLANKET
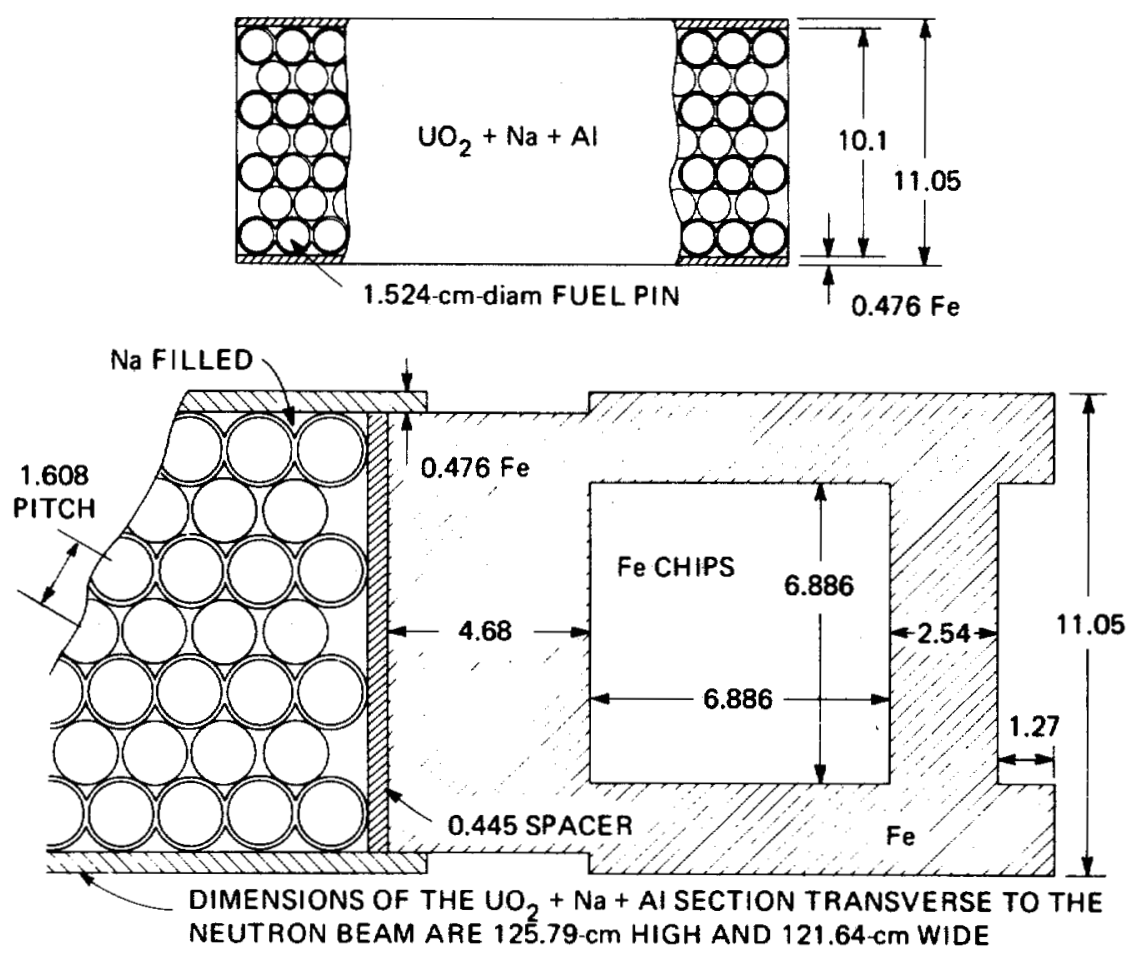

THEORETICAL DENSITY $=10.96 \mathrm{~g} / \mathrm{cc}$

ACTUAL DENSITY (0.94 THEO.) $=10.28 \mathrm{~g} / \mathrm{cc}$

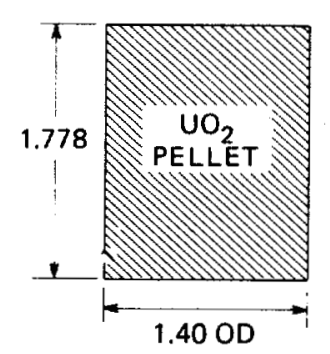
$1.40 \mathrm{OD}\left(\mathrm{UO}_{2}\right) \longrightarrow 0.010$

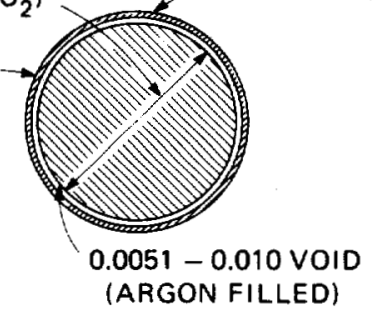

DIMENSIONS IN cm

Figure 2. Schematic of radial blanket slab containing $\mathrm{UO}_{2}$. 


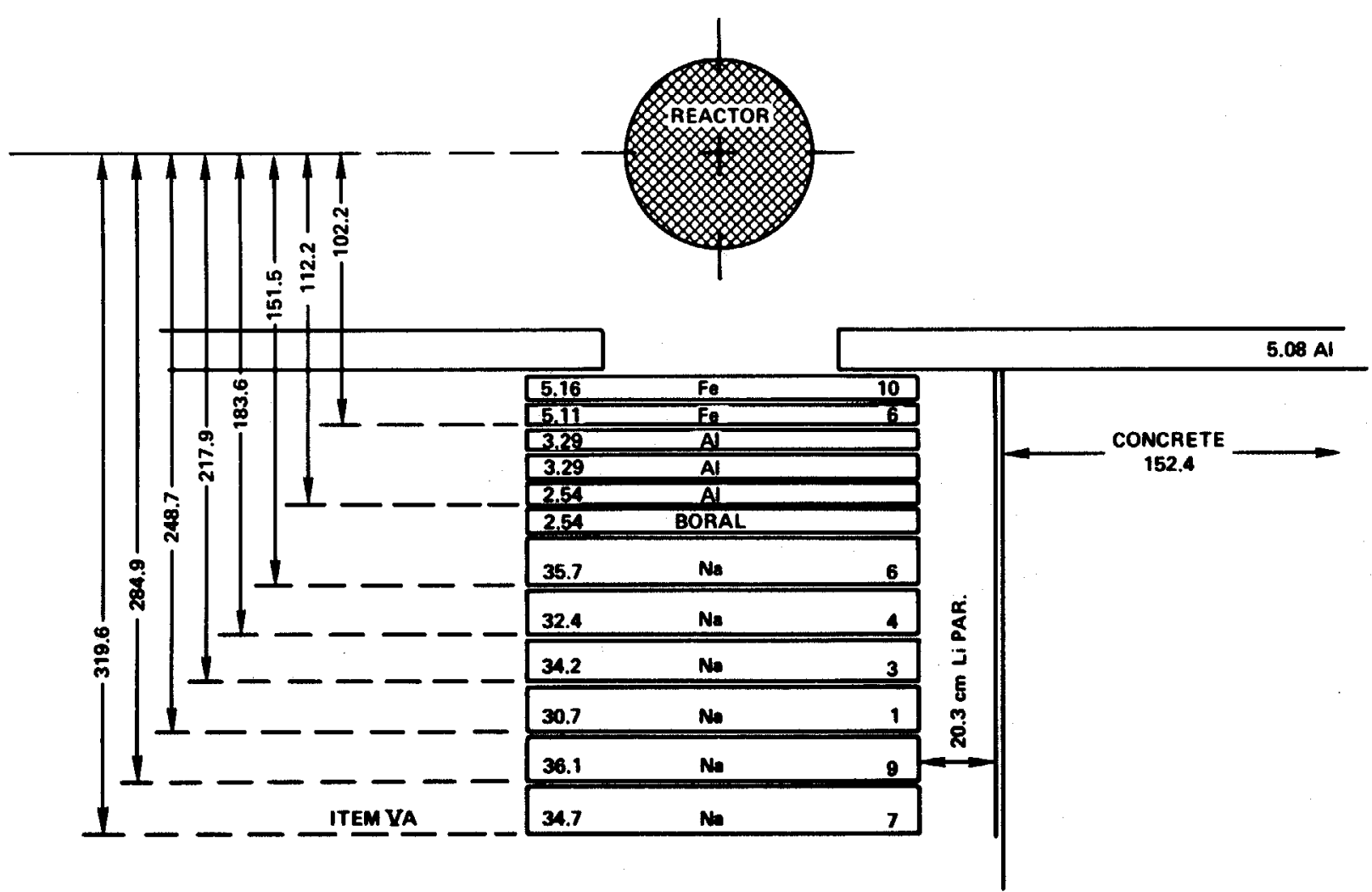

Figure 3. Schematic of iron, aluminum, boral and sodium spectrum modifier (SM2) (Item VA). Note: Lithiated paraffin covers lateral sides of configuration. 
ORNL-DWG 86-12606

\section{B, C CONTAINERS}
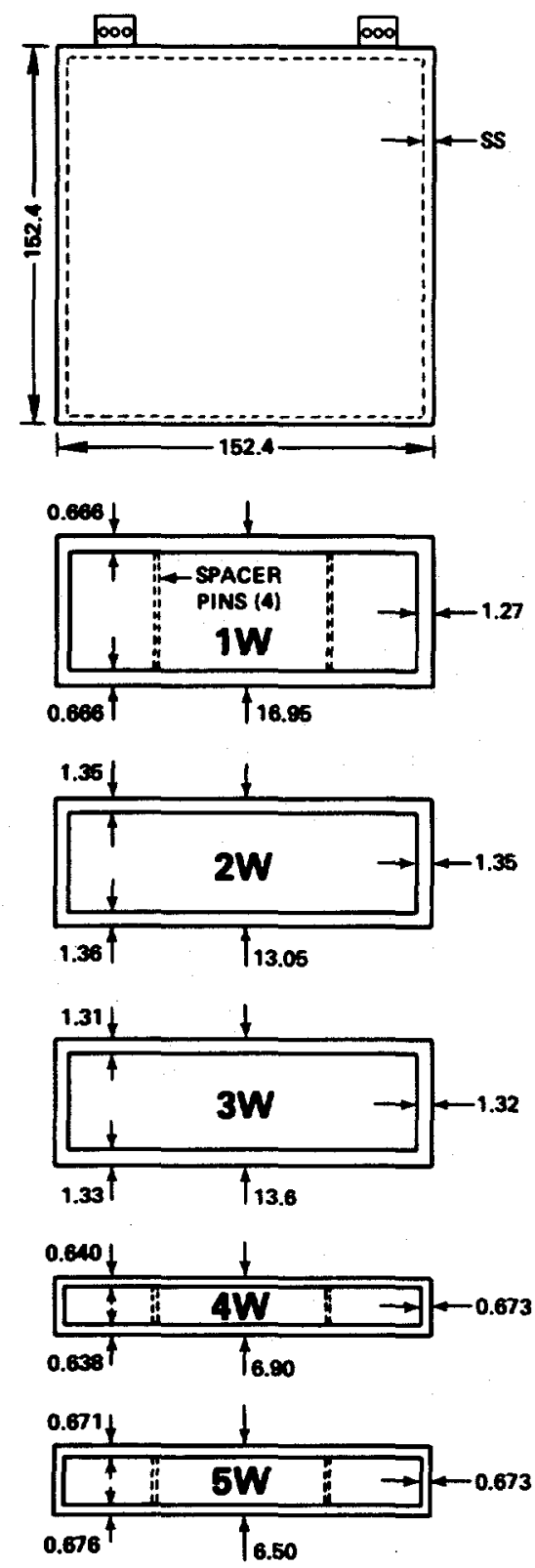

(ALL DIMENSIONS ARE IN CENTIMETERS)

Figure 4. Schematic of stainless steel containers used for boron carbide shield slabs. 


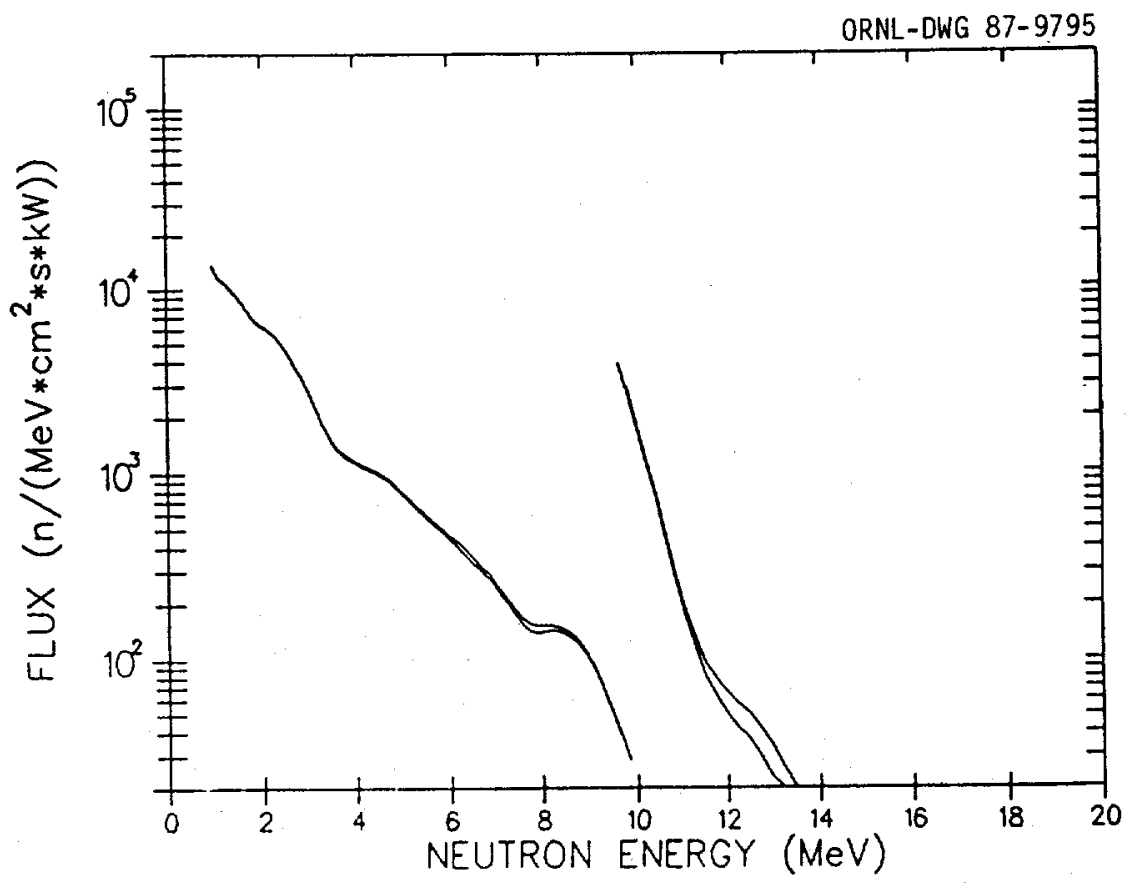

Pigure 5. Spectrum of high-energy neutrons $(>0.8 \mathrm{MeV})$ on centerline $178.8 \mathrm{~cm}$ beyond SMI (Item IA): Run 7856A.

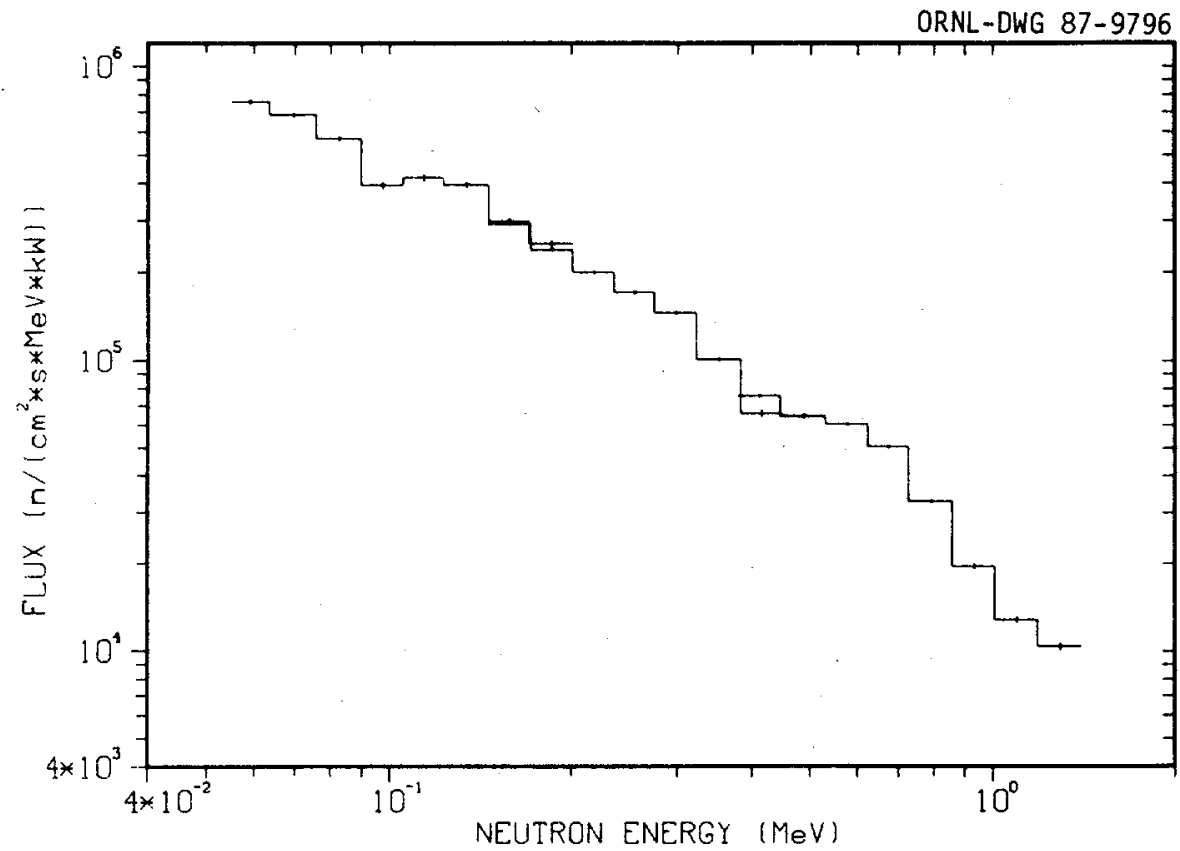

Figure 6. Neutron spectrum (50 keV to $1.4 \mathrm{MeV})$ on centerline 178.8 cm beyond the SM1 (Iten IA): Runs 1537C, 1537A, 1536A. 
号

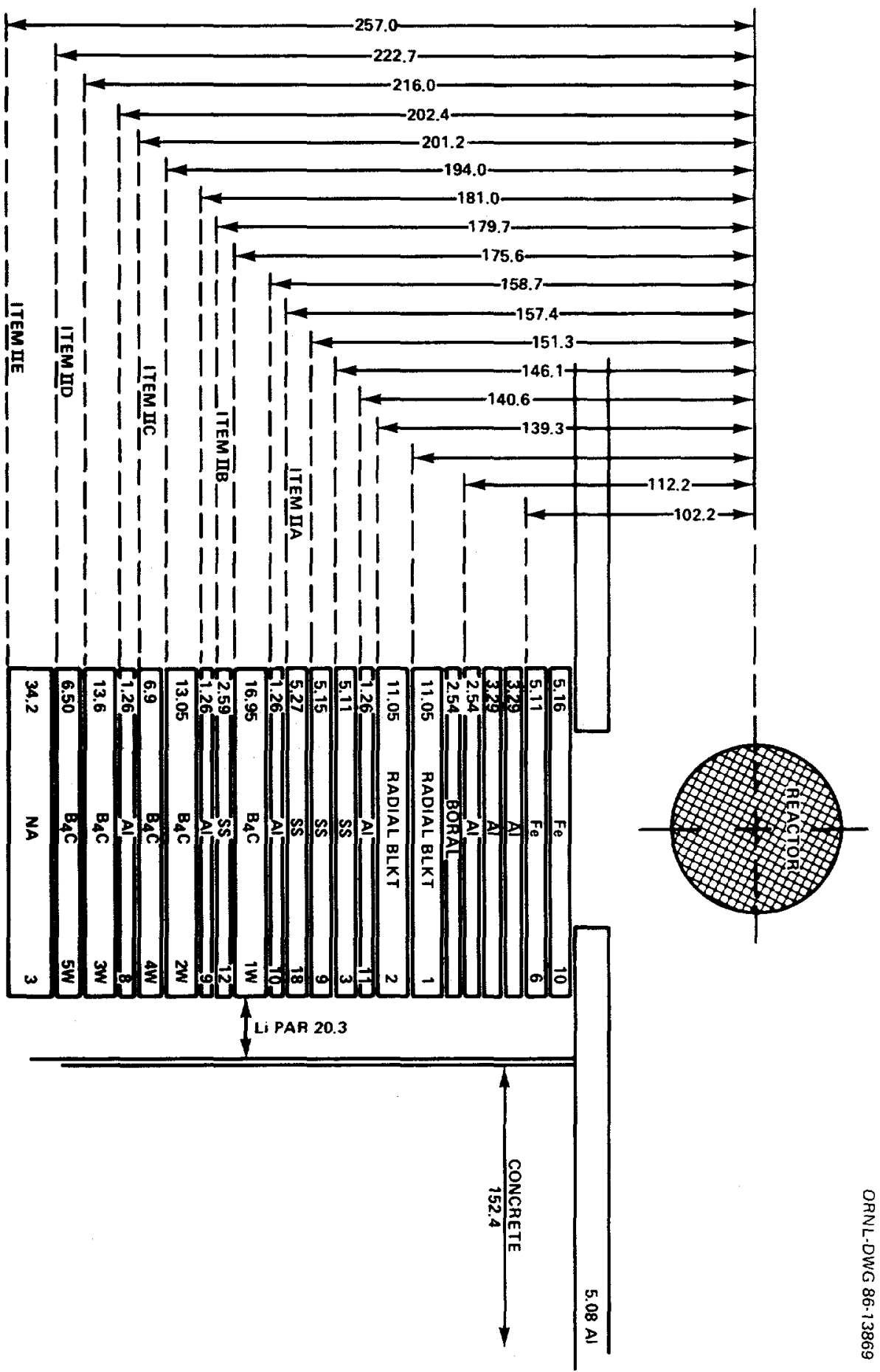




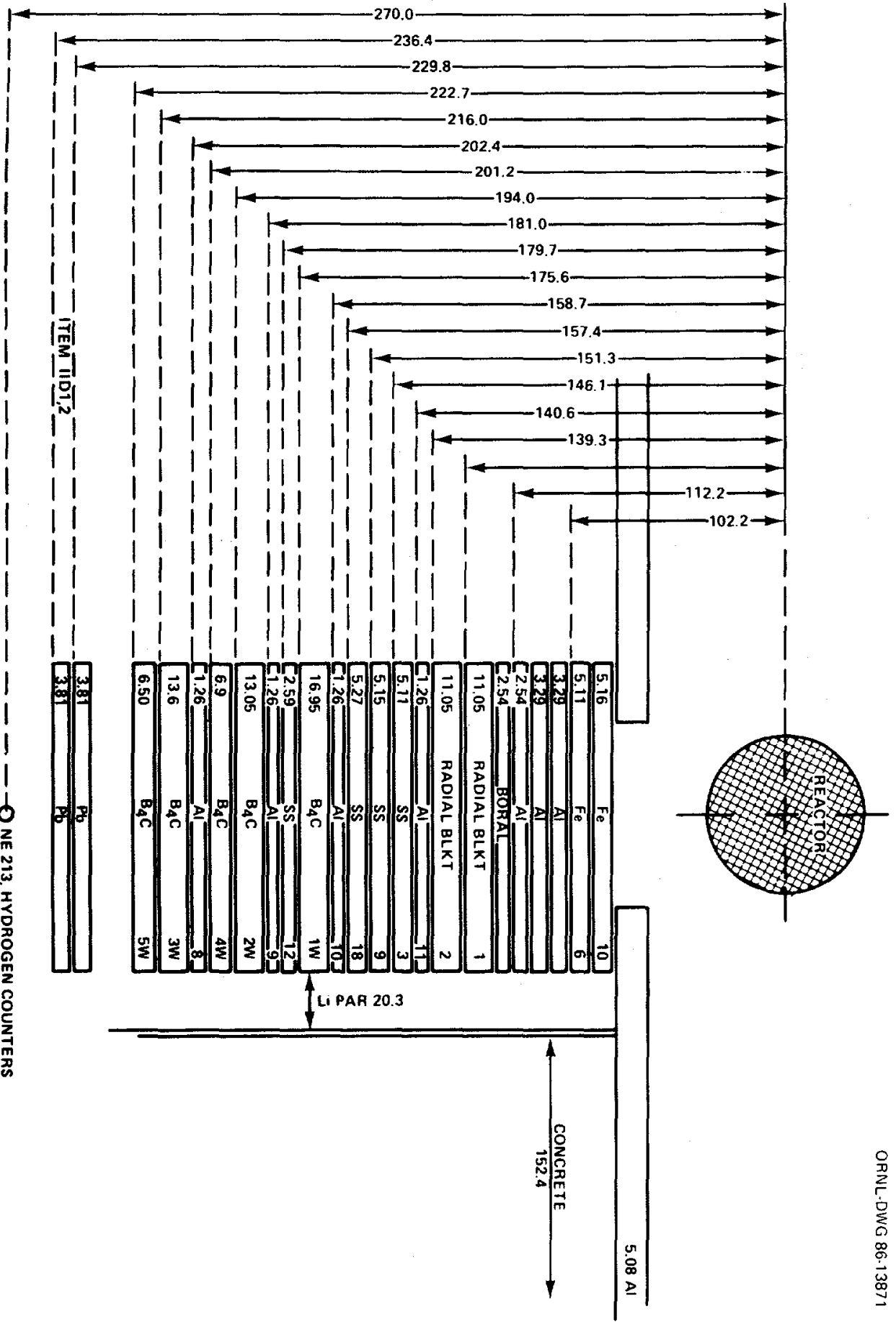




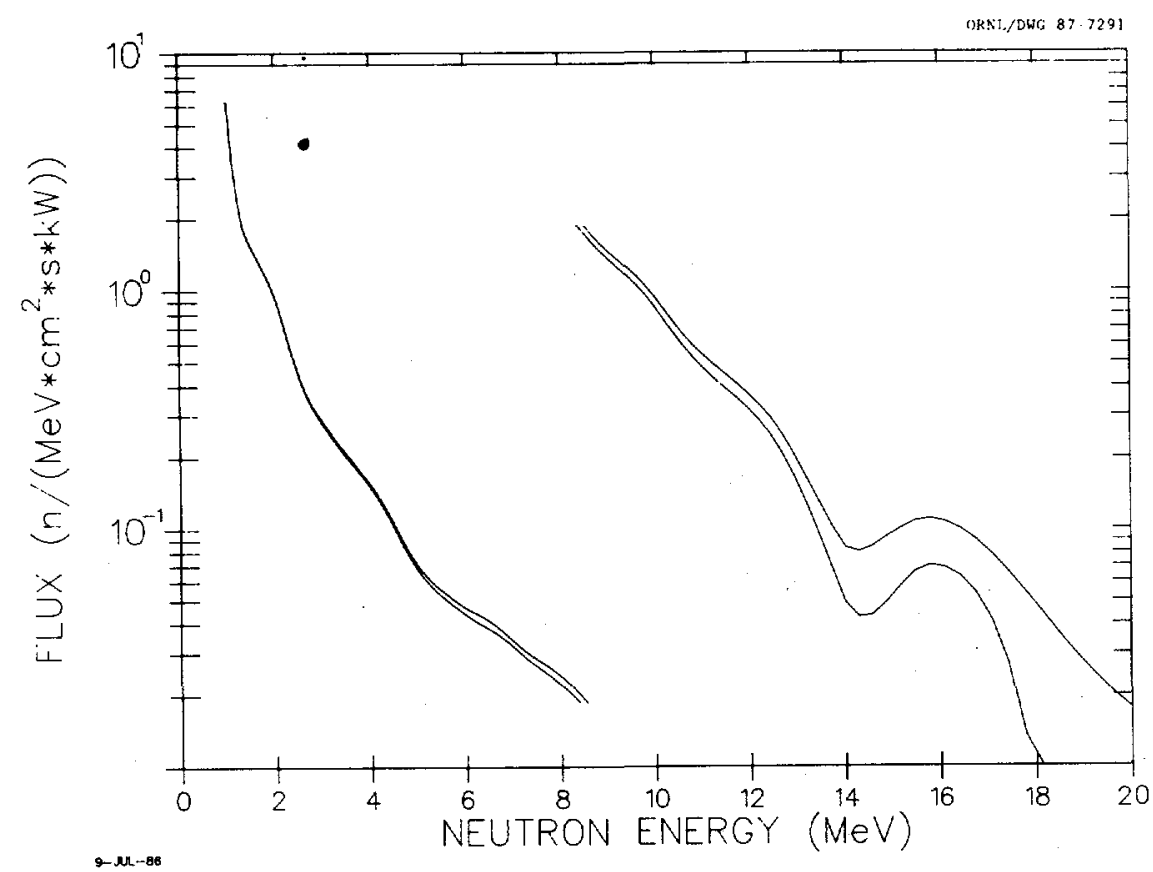

Figure 9. Spectrun of high-energy neutrons $(>0.8 \mathrm{MeV})$ on centerline $47.3 \mathrm{~cm}$ beyond $\mathrm{B}_{4} \mathrm{C}(33.6 \mathrm{~cm}$ beyond $\mathrm{Pb})$ (Item IID): Run 7857A.

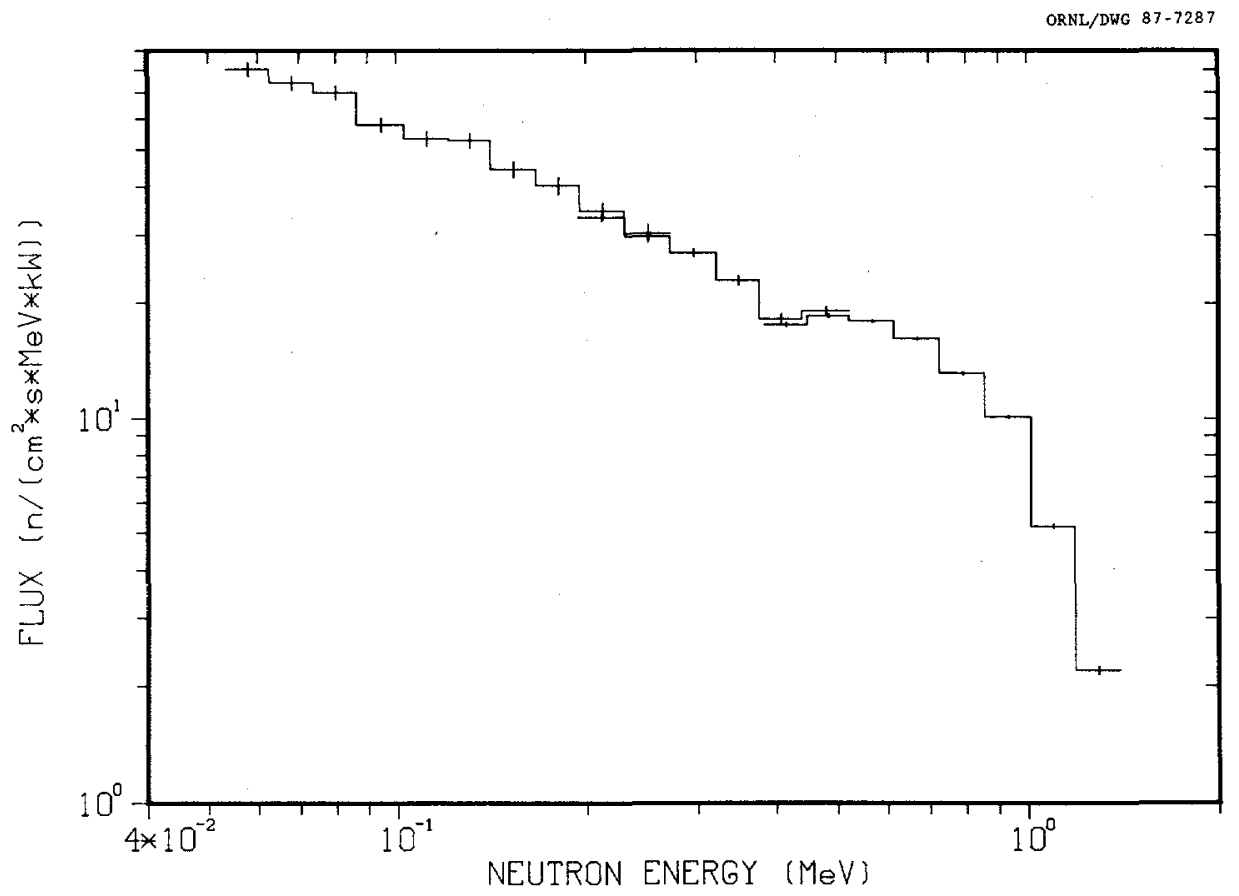

Figure 10. Neutron spectrum (50 keV to $1.4 \mathrm{MeV}$ ) on centerline 47.3 $\mathrm{cm}$ beyond $\mathrm{B}_{4} \mathrm{C}(33.6 \mathrm{~cm}$ beyond $\mathrm{Pb})$ (Item IID): Runs 1540A, 1539B, 1539A. 


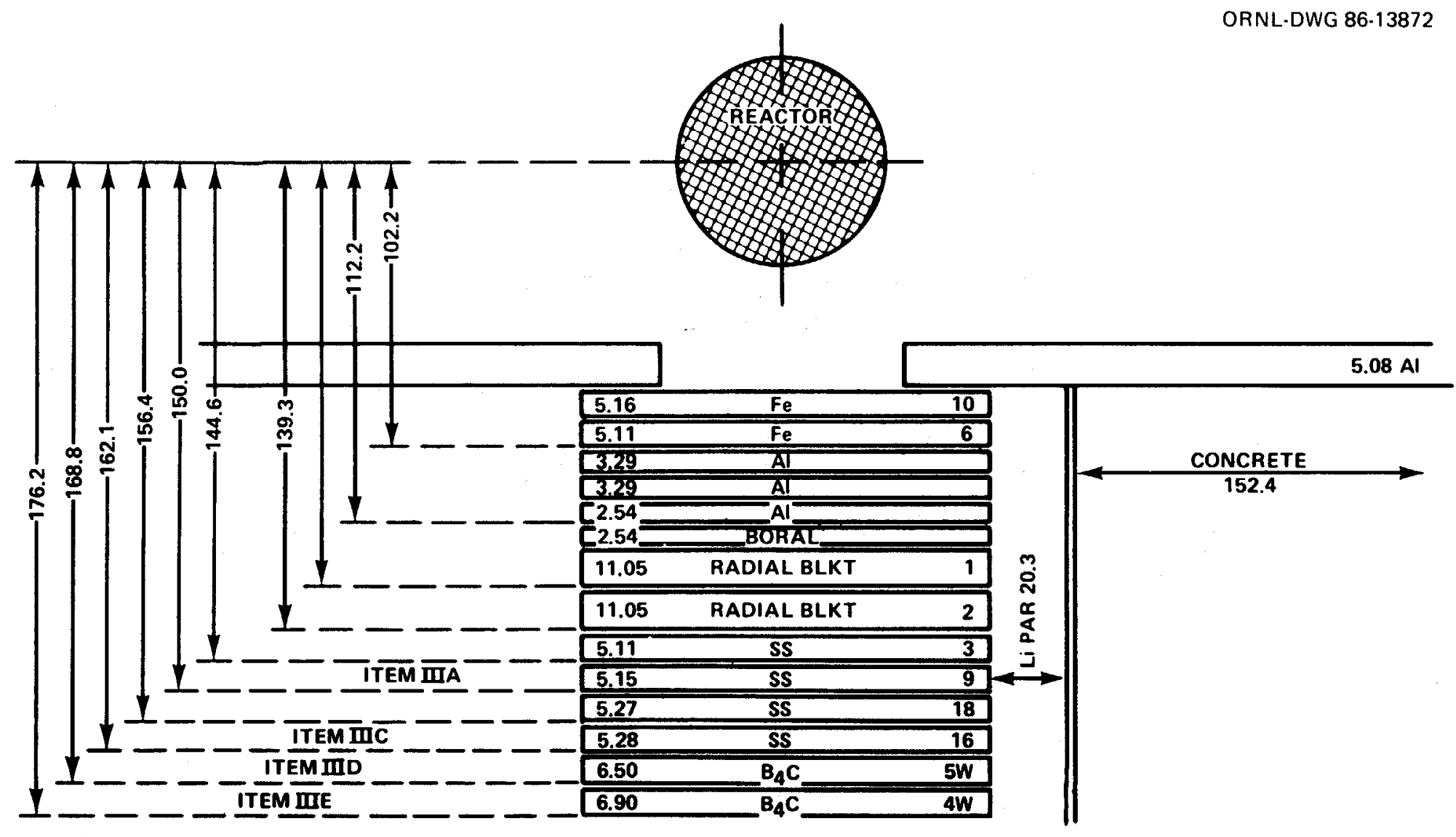

Figure 11. Schematic of SM1 plus shield configurations for Items IIIA, C, D, E. Note: Lithiated paraffin covers lateral sides of configuration. 

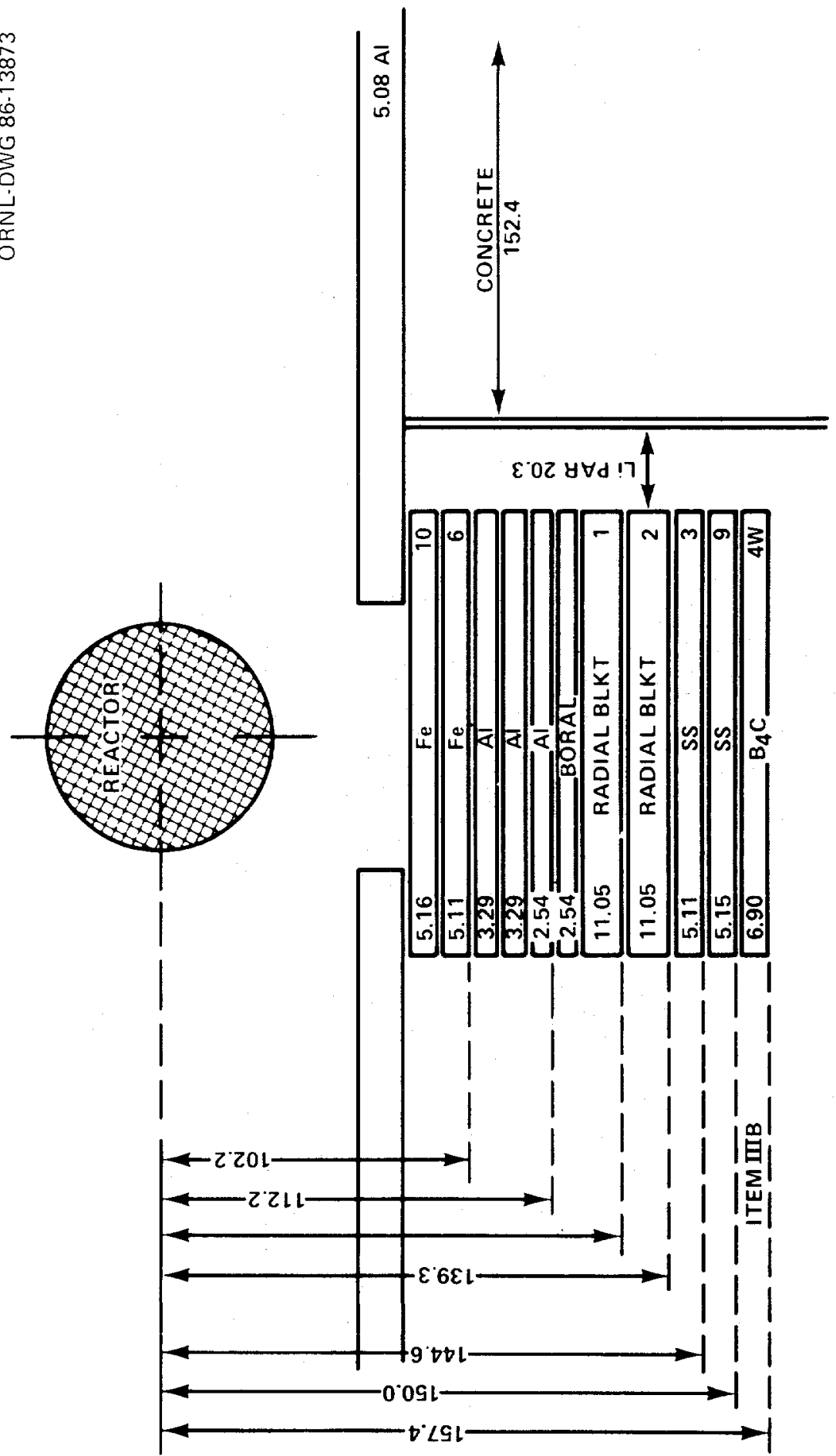

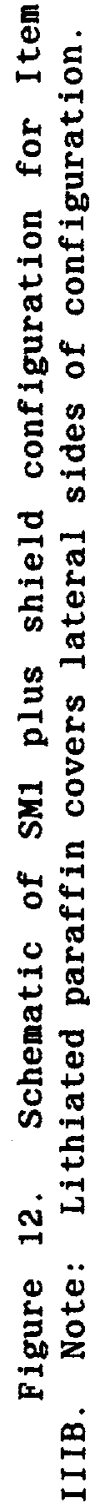


9
$\frac{9}{1}$
$\vdots$
0
0
$\vdots$
$\vdots$
$\vdots$
$\vdots$
0
0
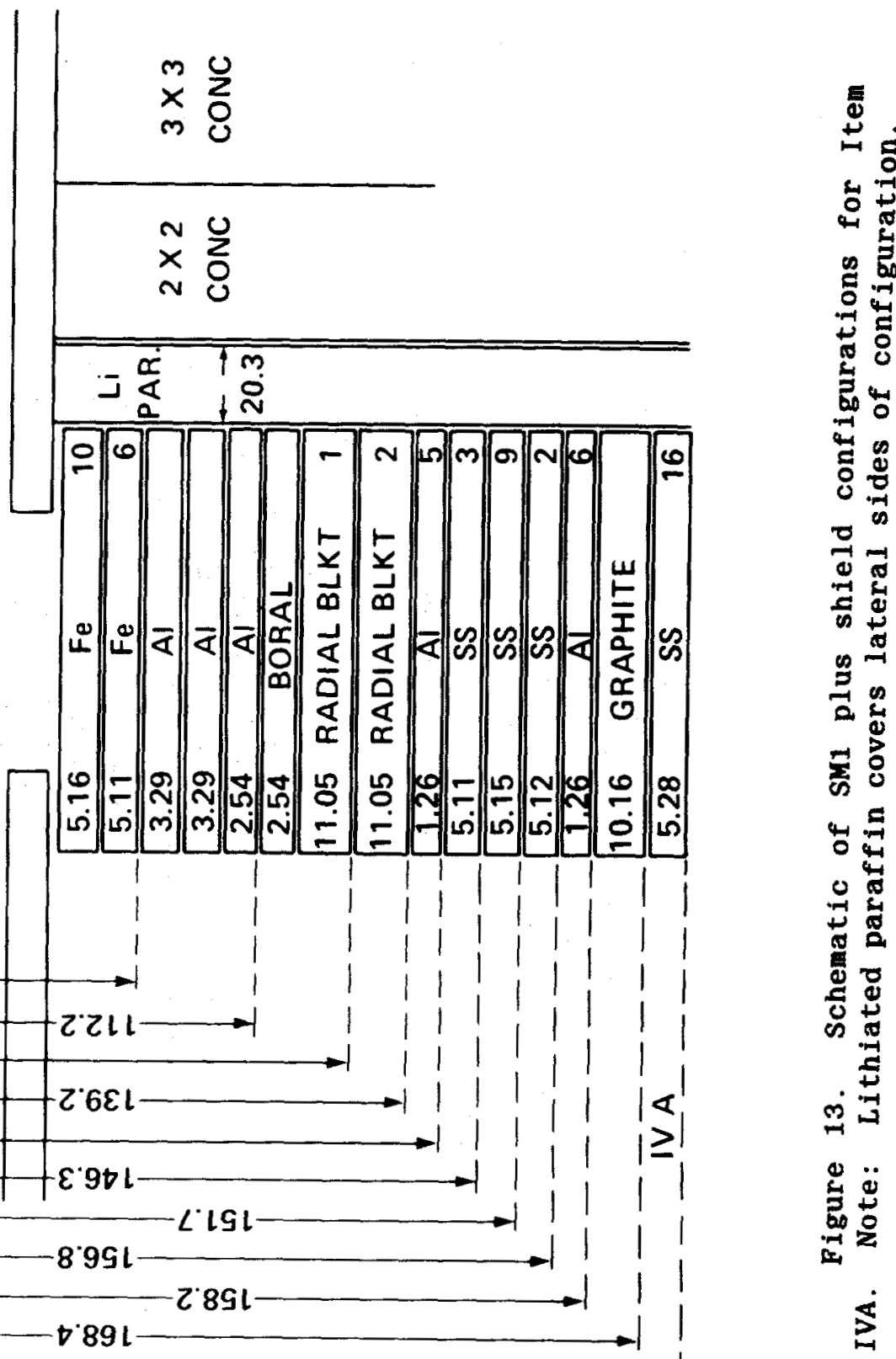
8
0
0
0
0
0
$\vdots$
0
$ن$
$\vdots$
0
0

$\begin{array}{ll}m & 0 \\ \times & 2 \\ m & 0\end{array}$

$N$
$\times$
$N$
0

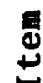

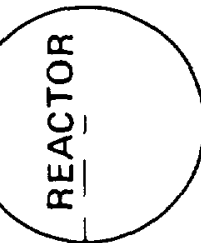

呫

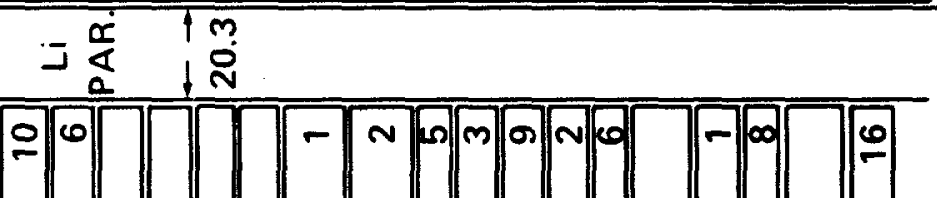

o

5.

車

至

幽

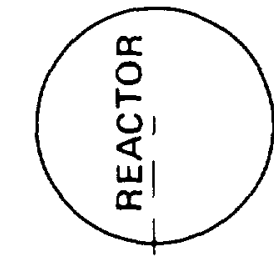

昱

$\infty$

过

$\because$ =

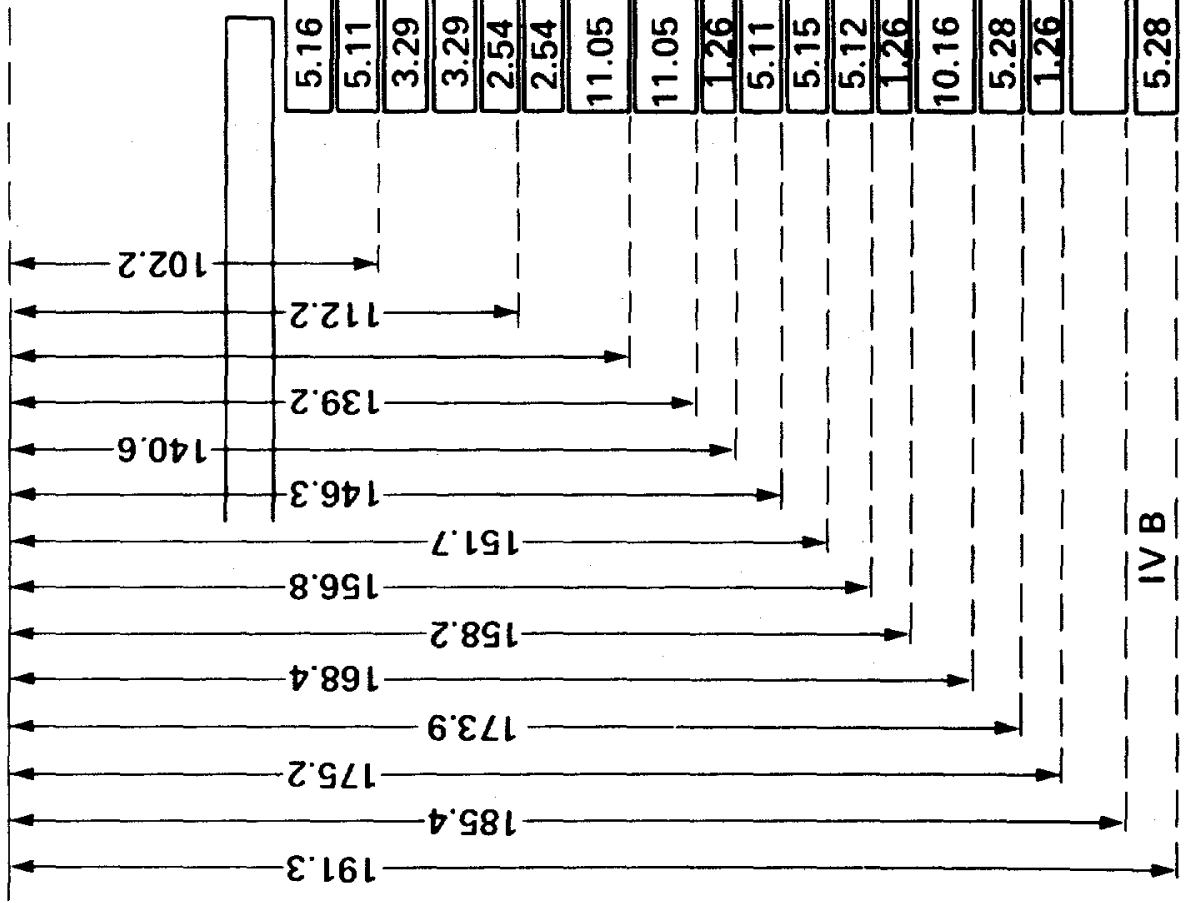




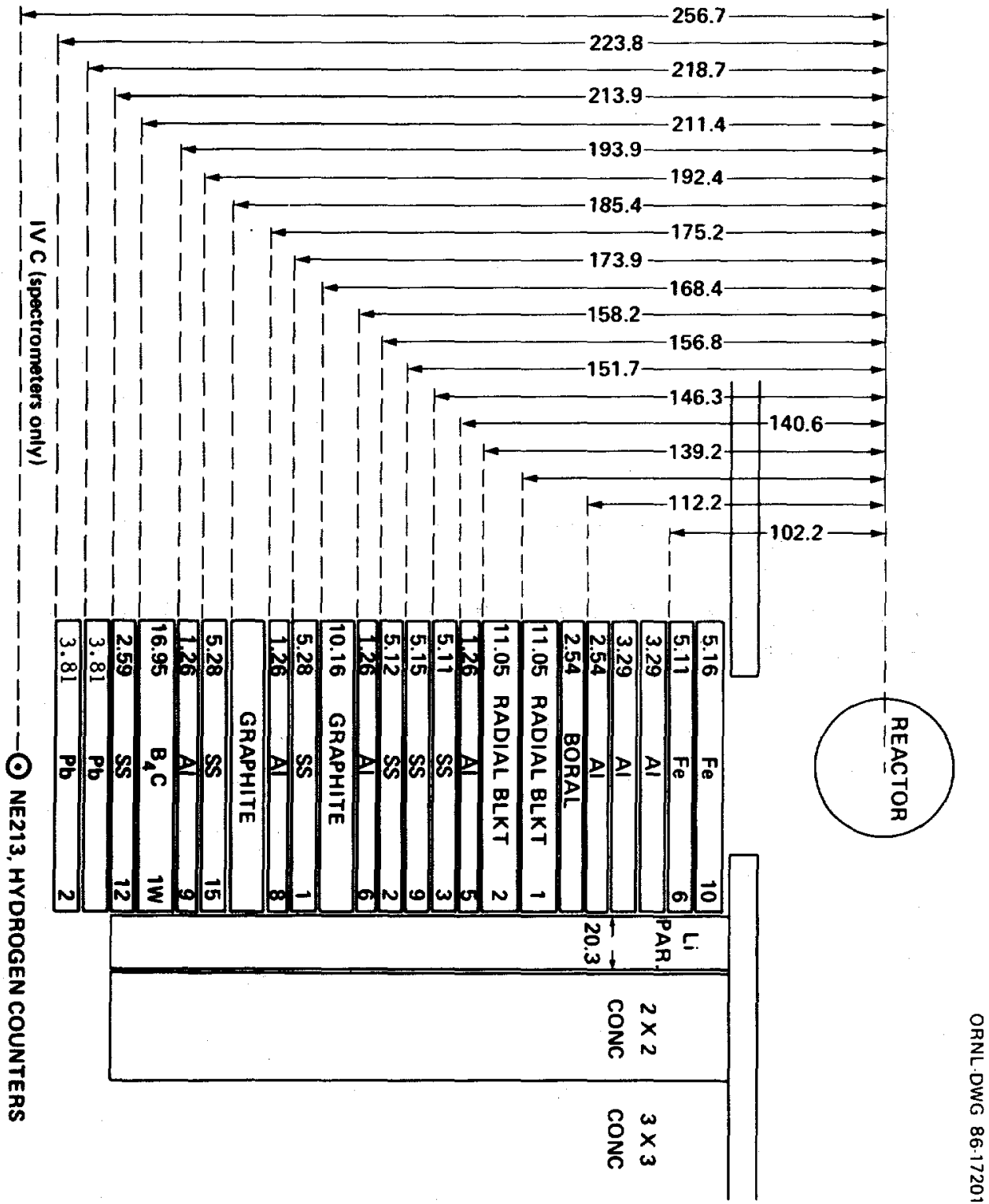




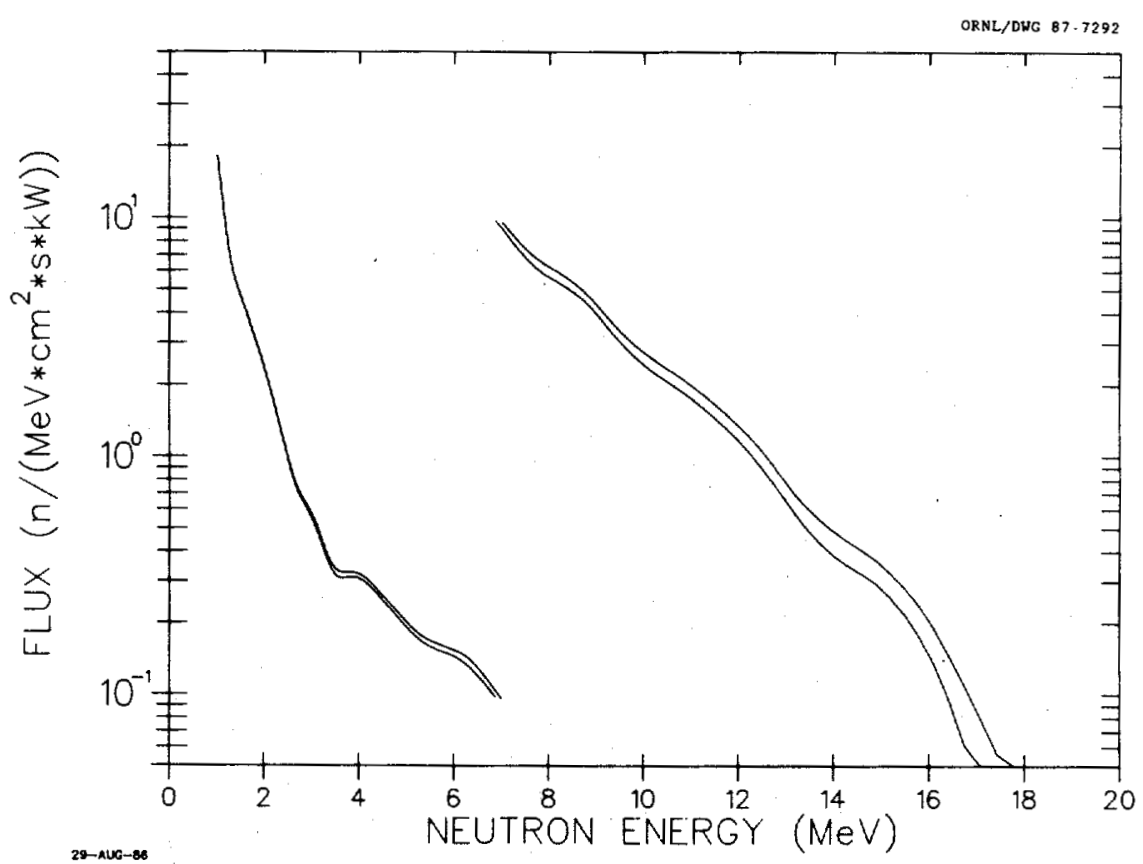

Figure 16. Spectrun of high-energy neutrons (>0.8 MeV) on centerline $42.8 \mathrm{~cm}$ beyond SS ( $32.9 \mathrm{~cm}$ beyond $\mathrm{Pb}$ ) (Item IVC): Run 7860A.

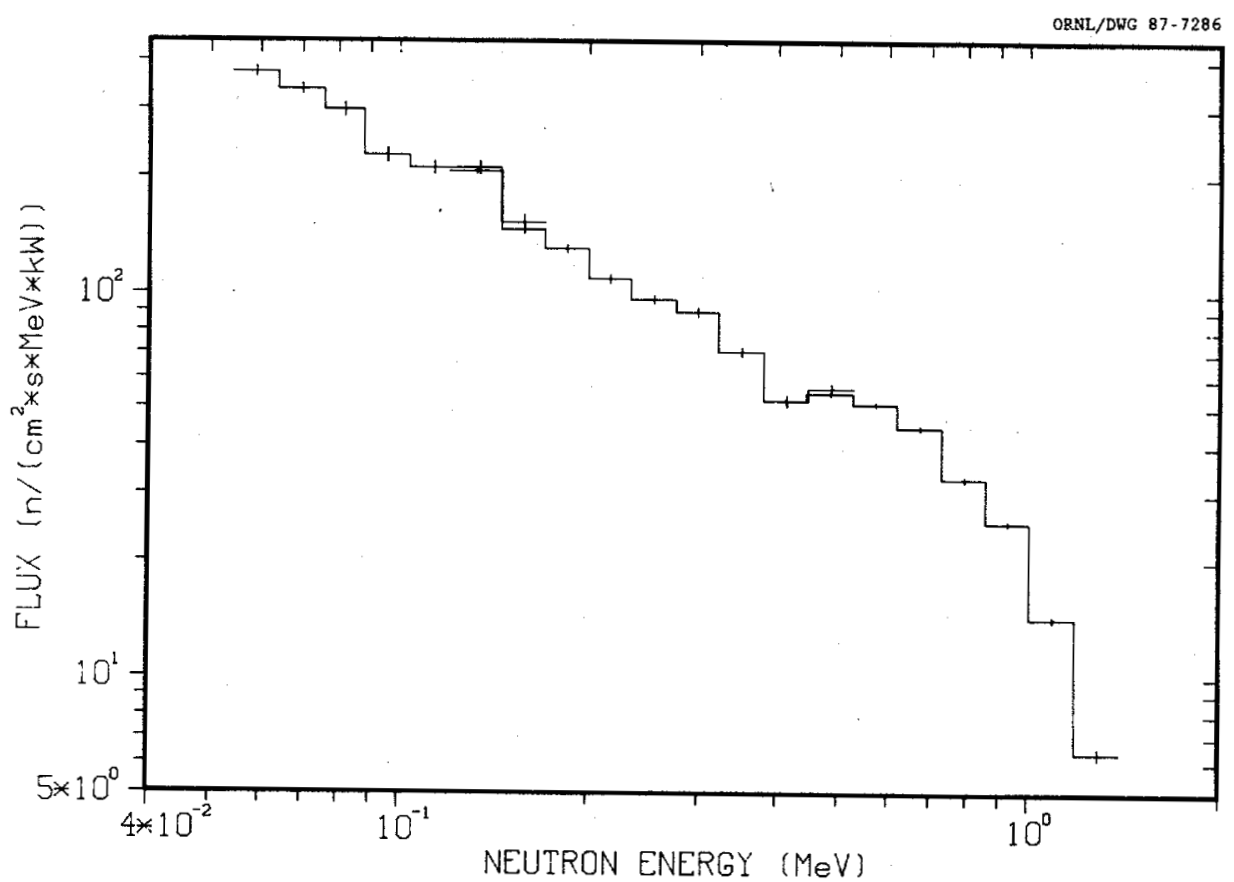

Figure 17. Neutron spectrum (50 keV to $1.4 \mathrm{MeV}$ ) on centerline 42.8 ca beyond Ss (32.9 cn beyond $\mathrm{Pb}$ ) (Iten IVC): Runs 1546A, 1546B, 1547A. 


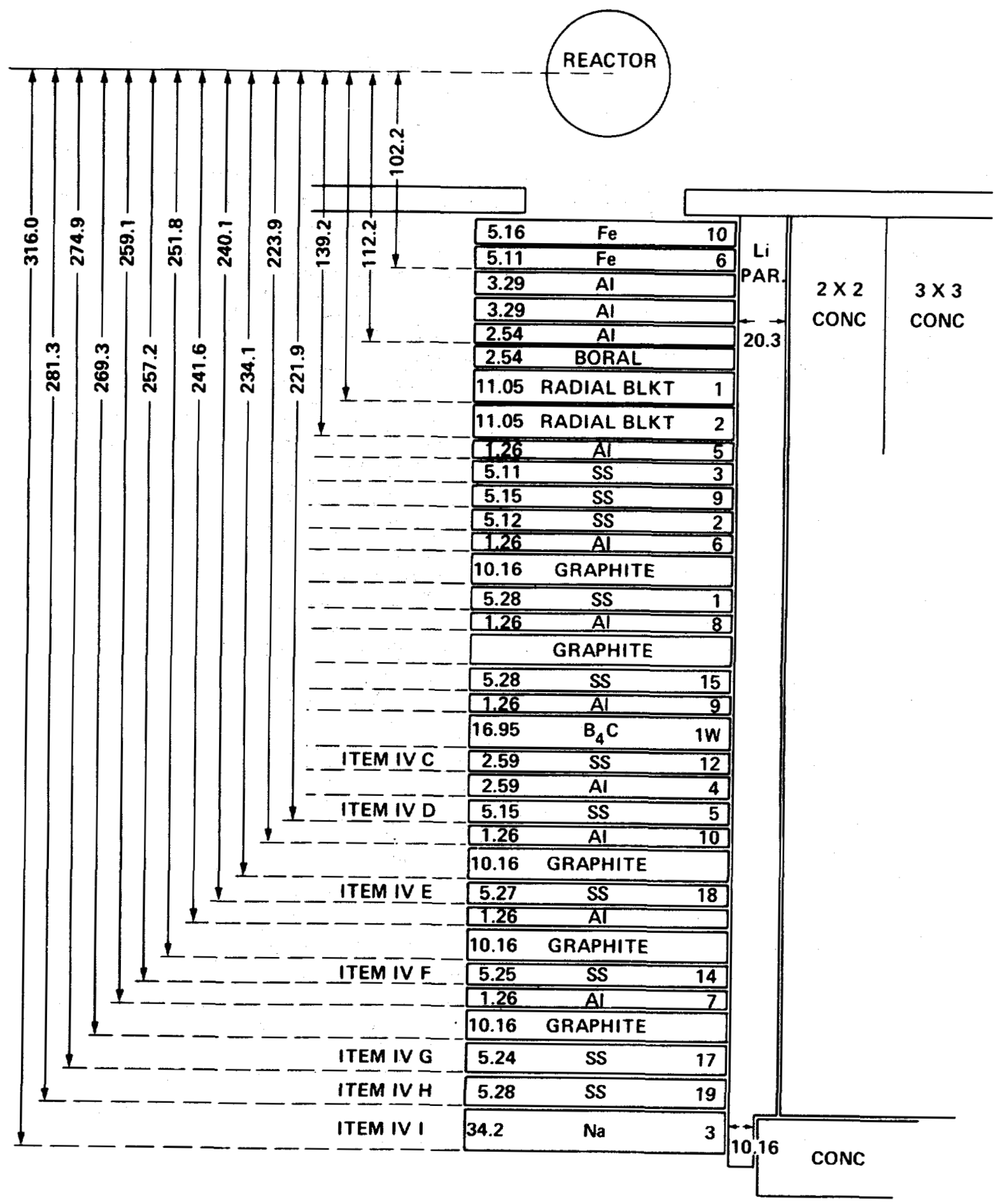

Figure 18. Schematic of SM1 plus shield configurations for Items IVC-I. Note: Lithiated parafin covers lateral sides of configuration. 


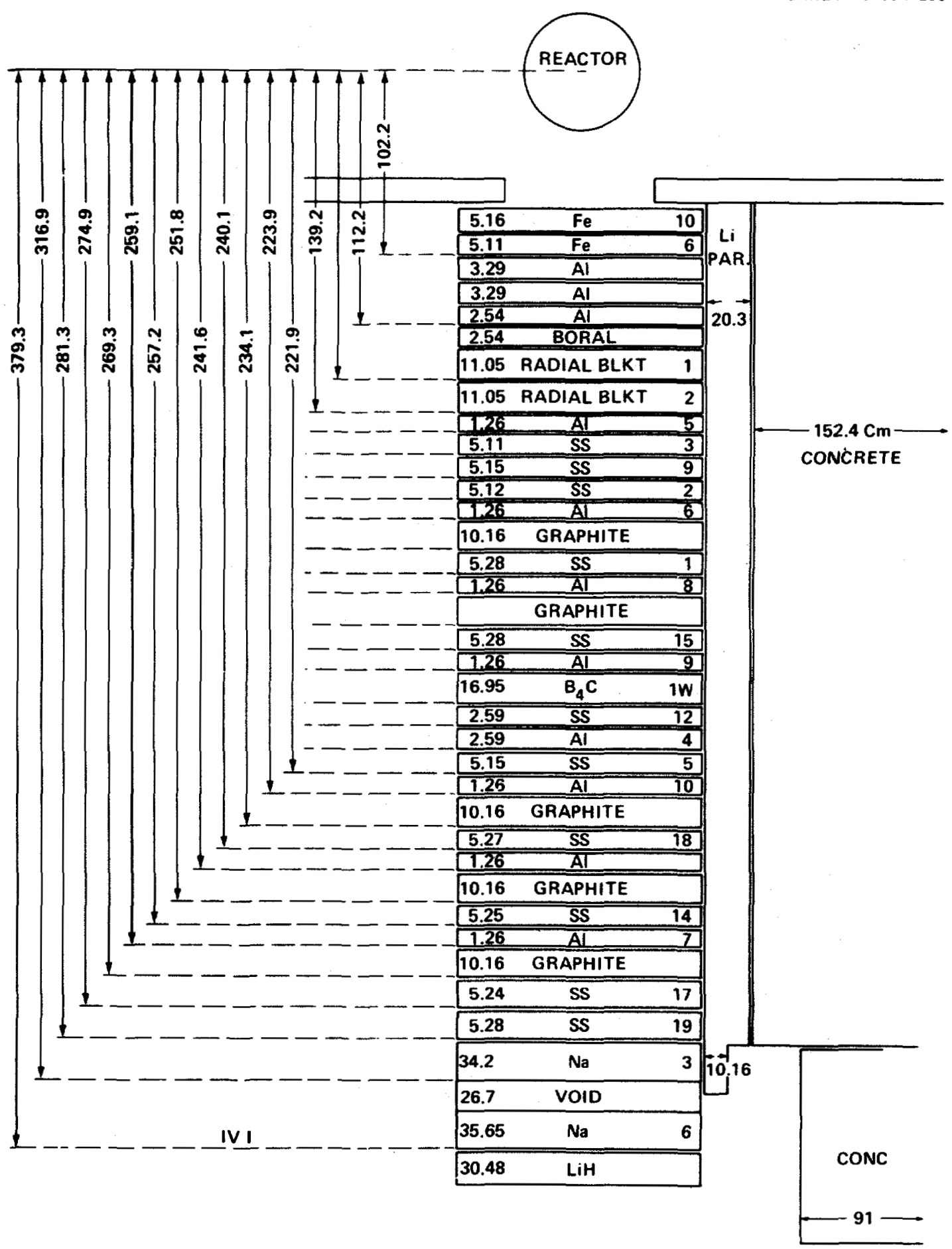

Figure 19. Schematic of SMI plus shield configuration for Item IVIA. Note: Lithiated parafin covers lateral sides of configuration. 


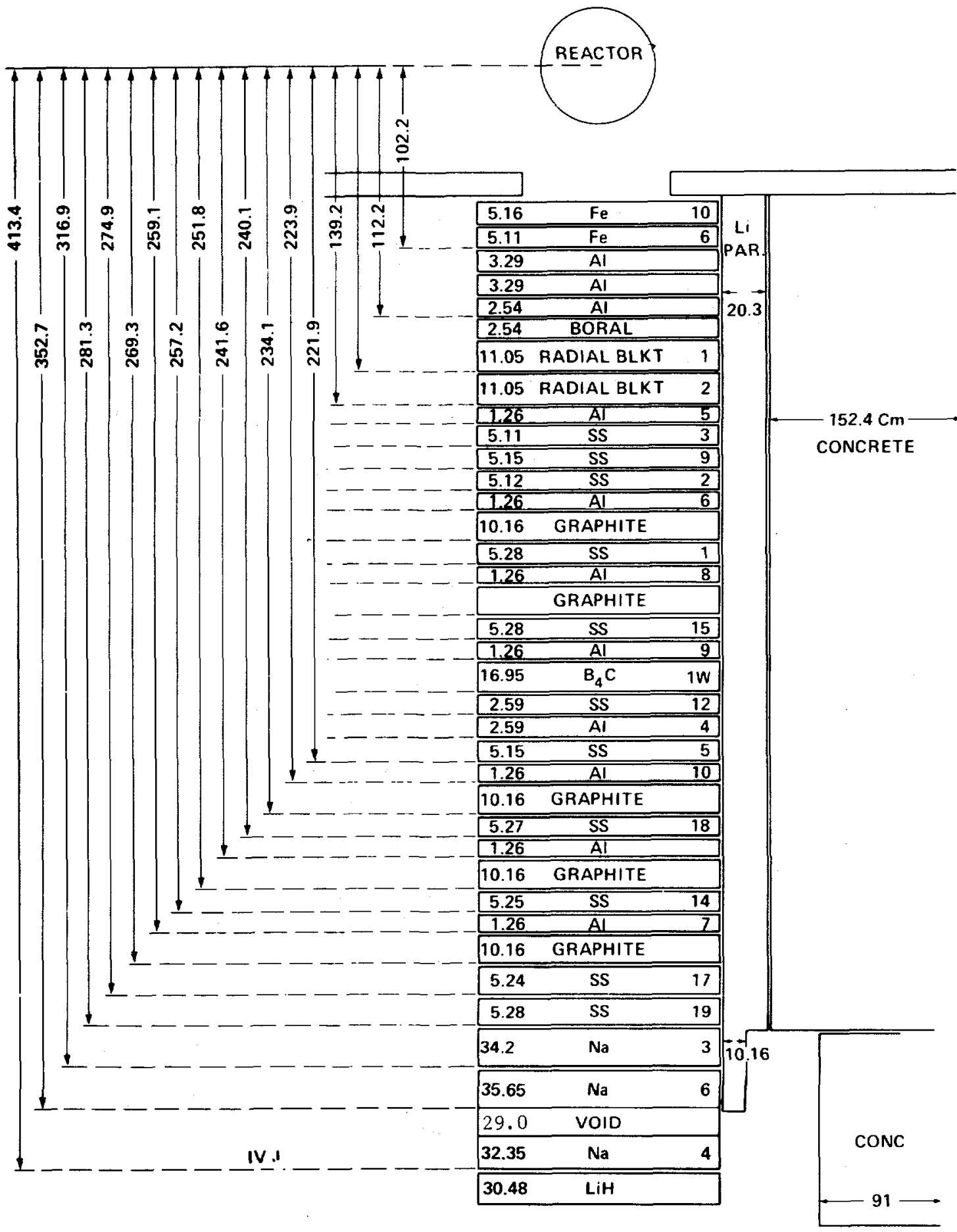

Figure 20. Schematic of SM1 plus shield configuration for Item IVJ. Note: Lithiated paraffin covers lateral sides of configuration. 
ORNL.DWG 86.17204

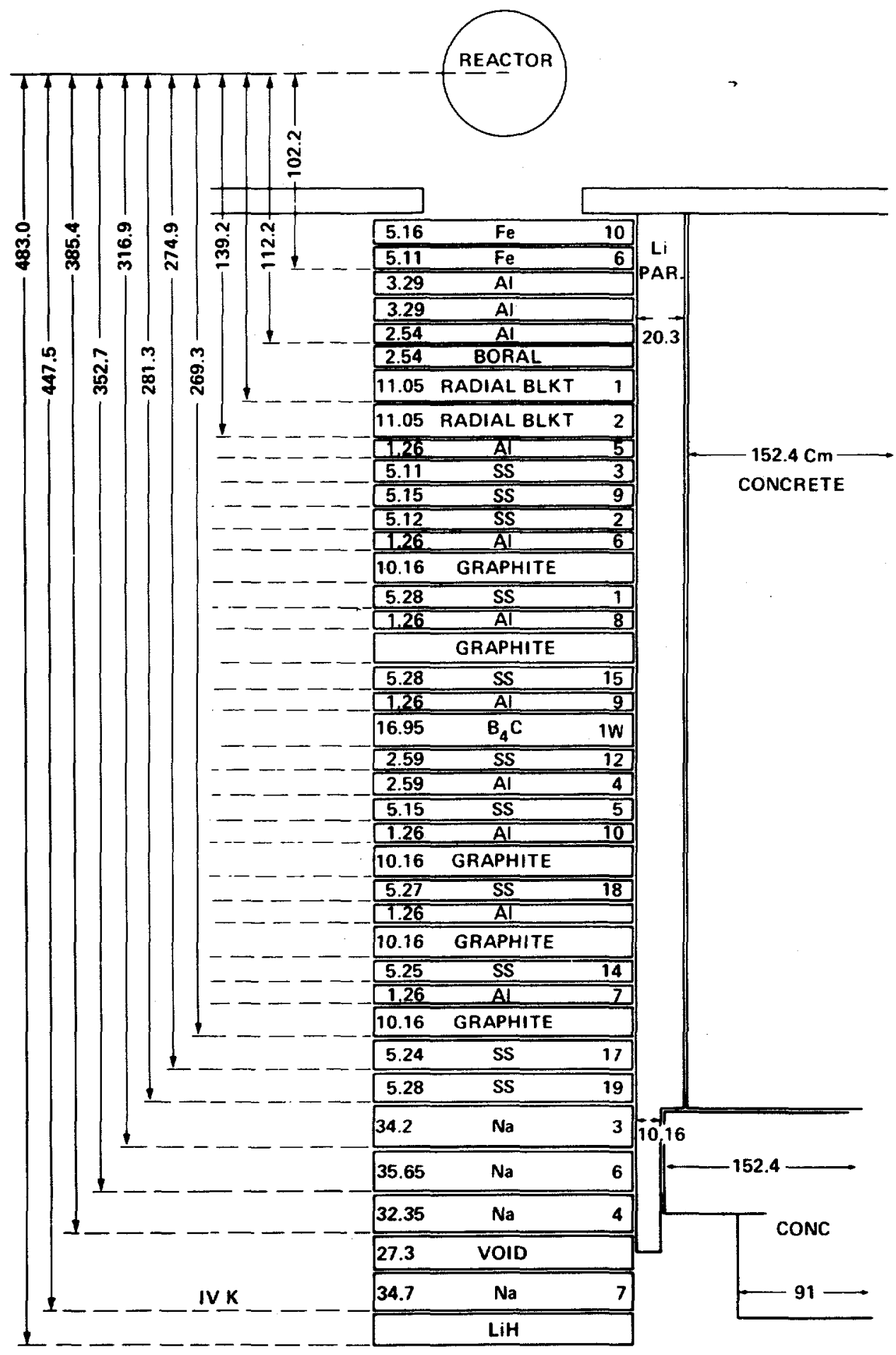

Figure 21. Schematic of SM1 plus shield configuration for IVK. Note: Lithiated paraffin covers lateral sides of configuration. 


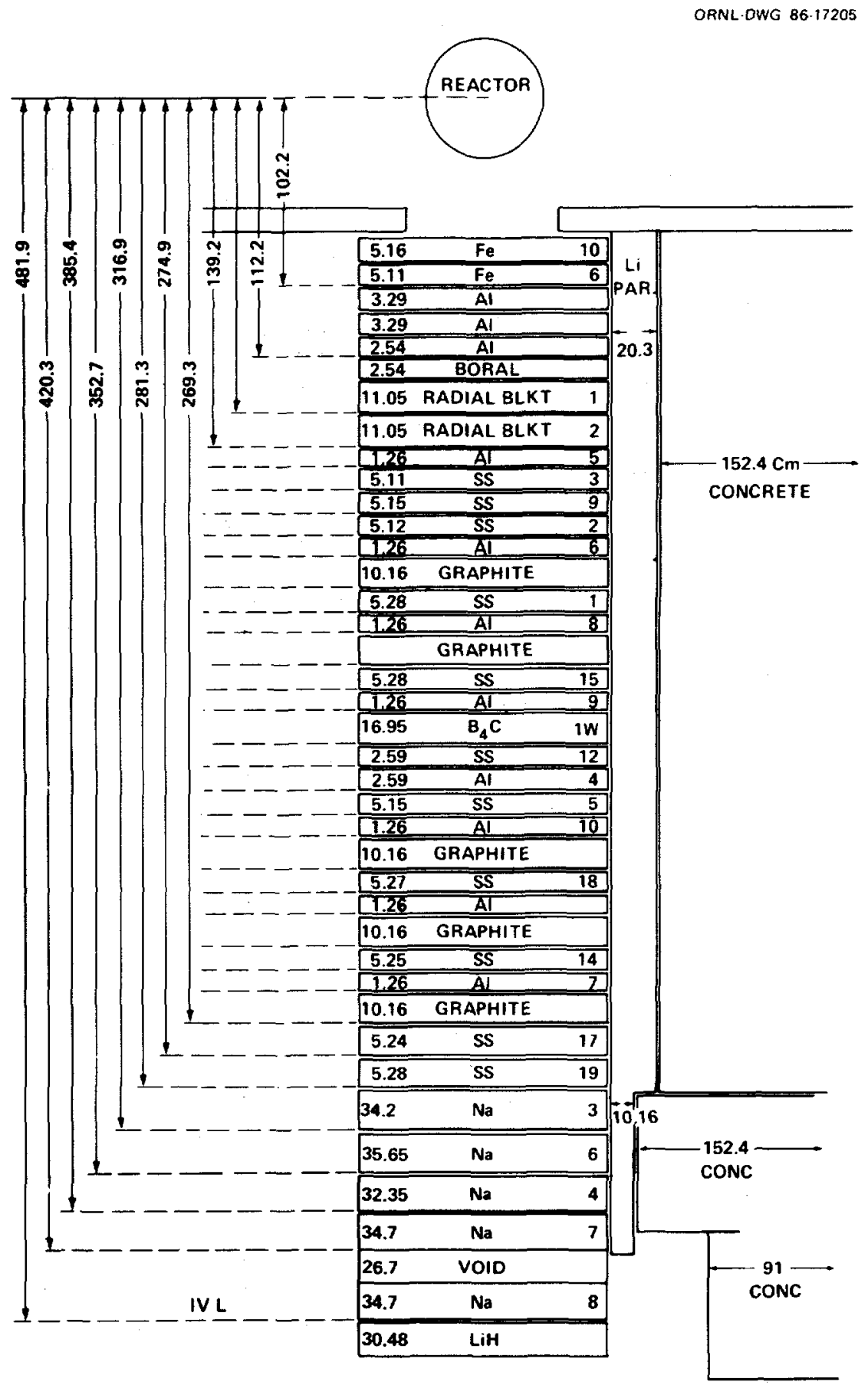

Figure 22. Schenatic of SM1 plus shield configuration for Item IVL. Note: Lithiated paraffin covers lateral sides of configuration. 
ORNL-DWG $86 \cdot 17202$

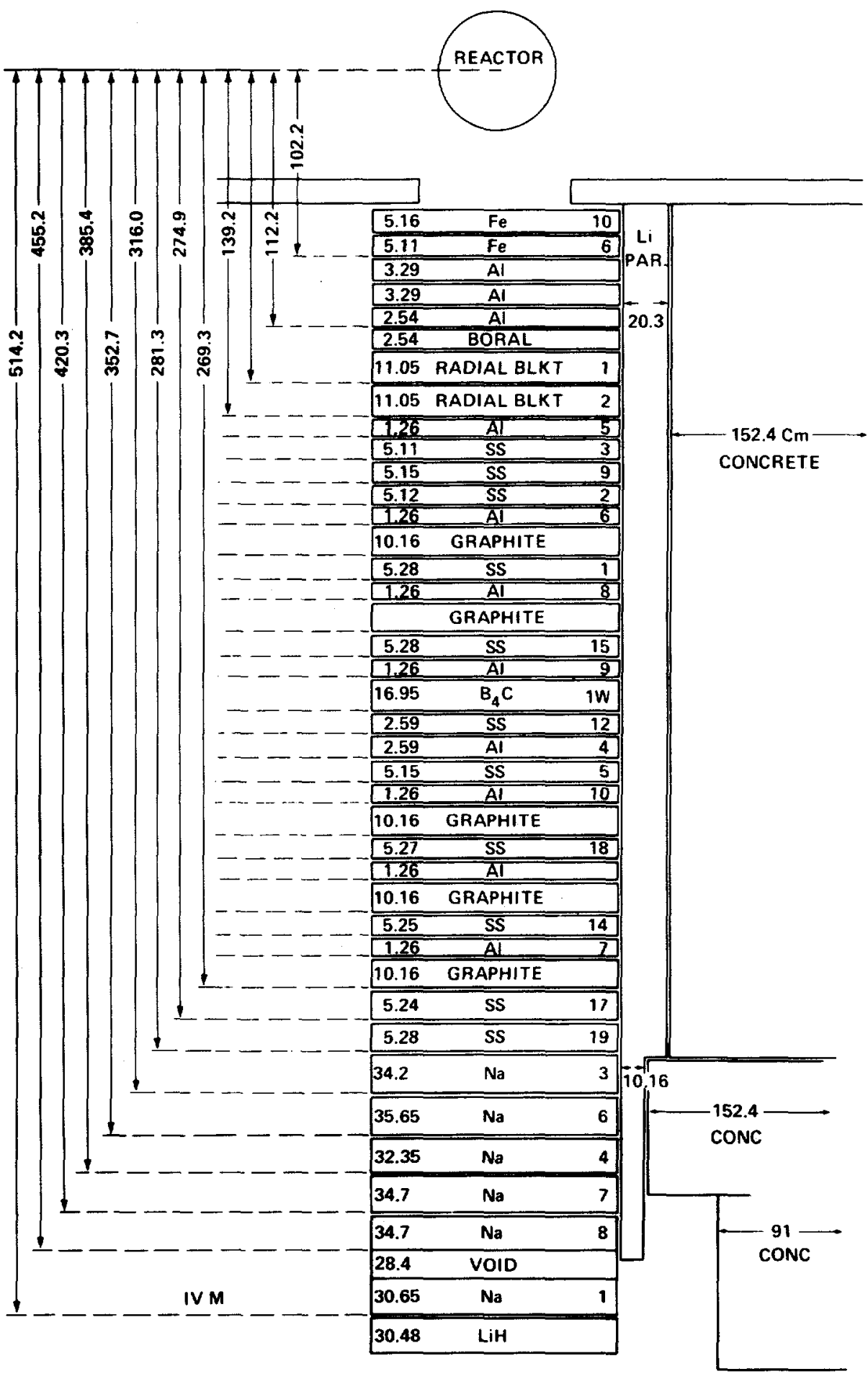

Figure 23. Schenatic of SM1 plus shield configuration for Item IVM. Note: Lithiated paraffin covers lateral sides of configuration. 


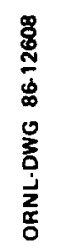
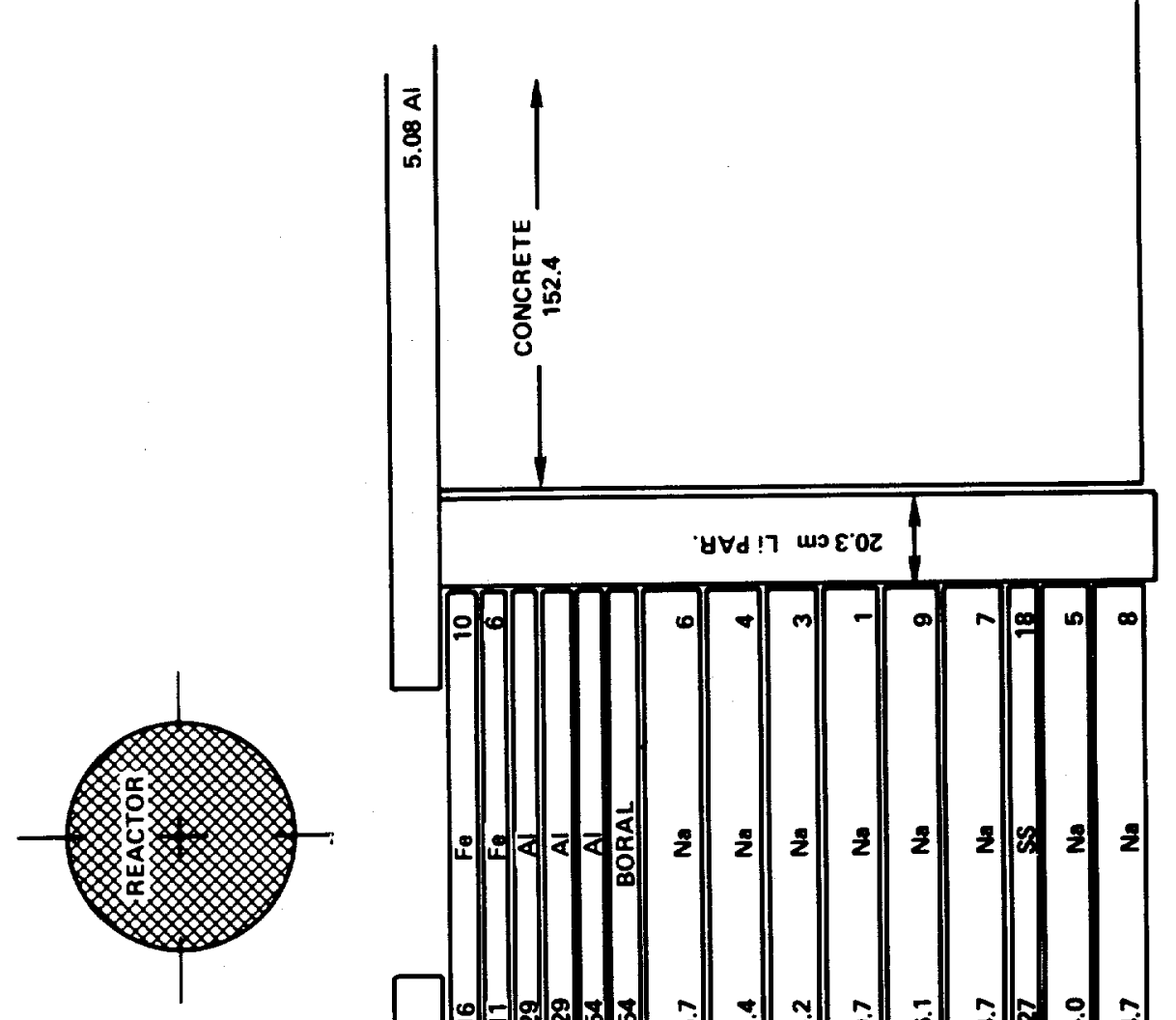

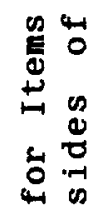

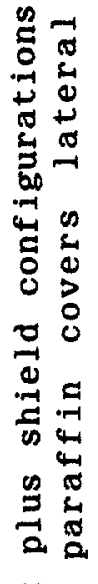

需。

4

这

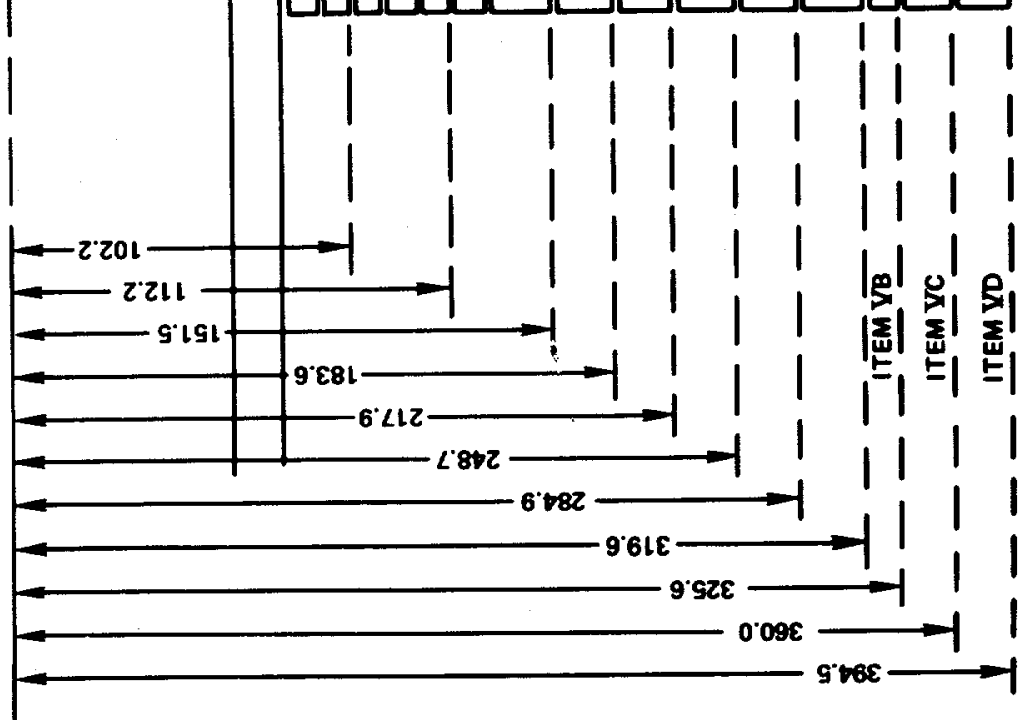

牙

异

ज़

ง

它先

昰 0 i

告 
ORNL-DWG 86-12609

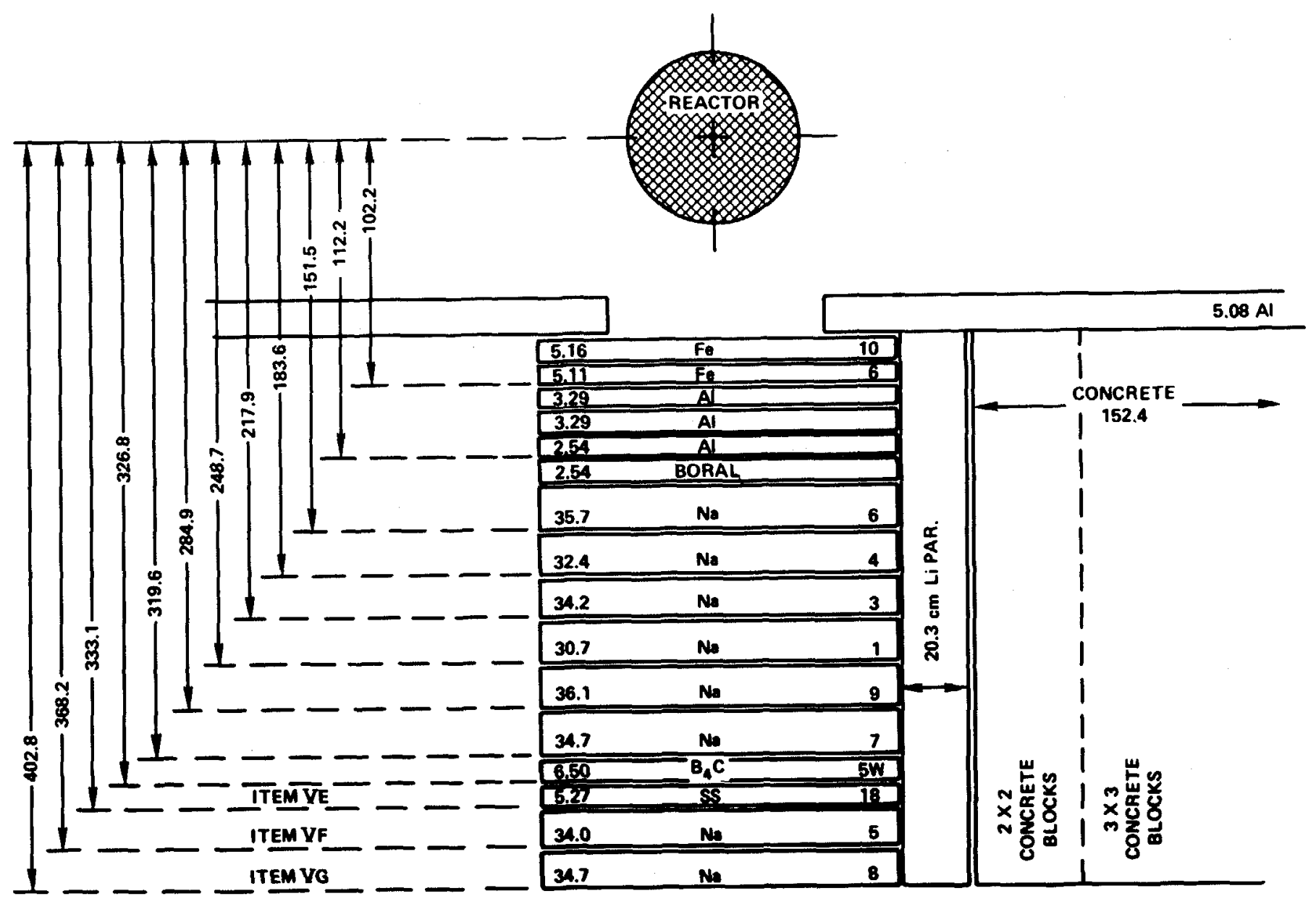

Figure 25. Schematic of SM2 plus shield configurations for Items VE, F, G. Note: Lithiated parafin covers lateral sides of conf igurations. 


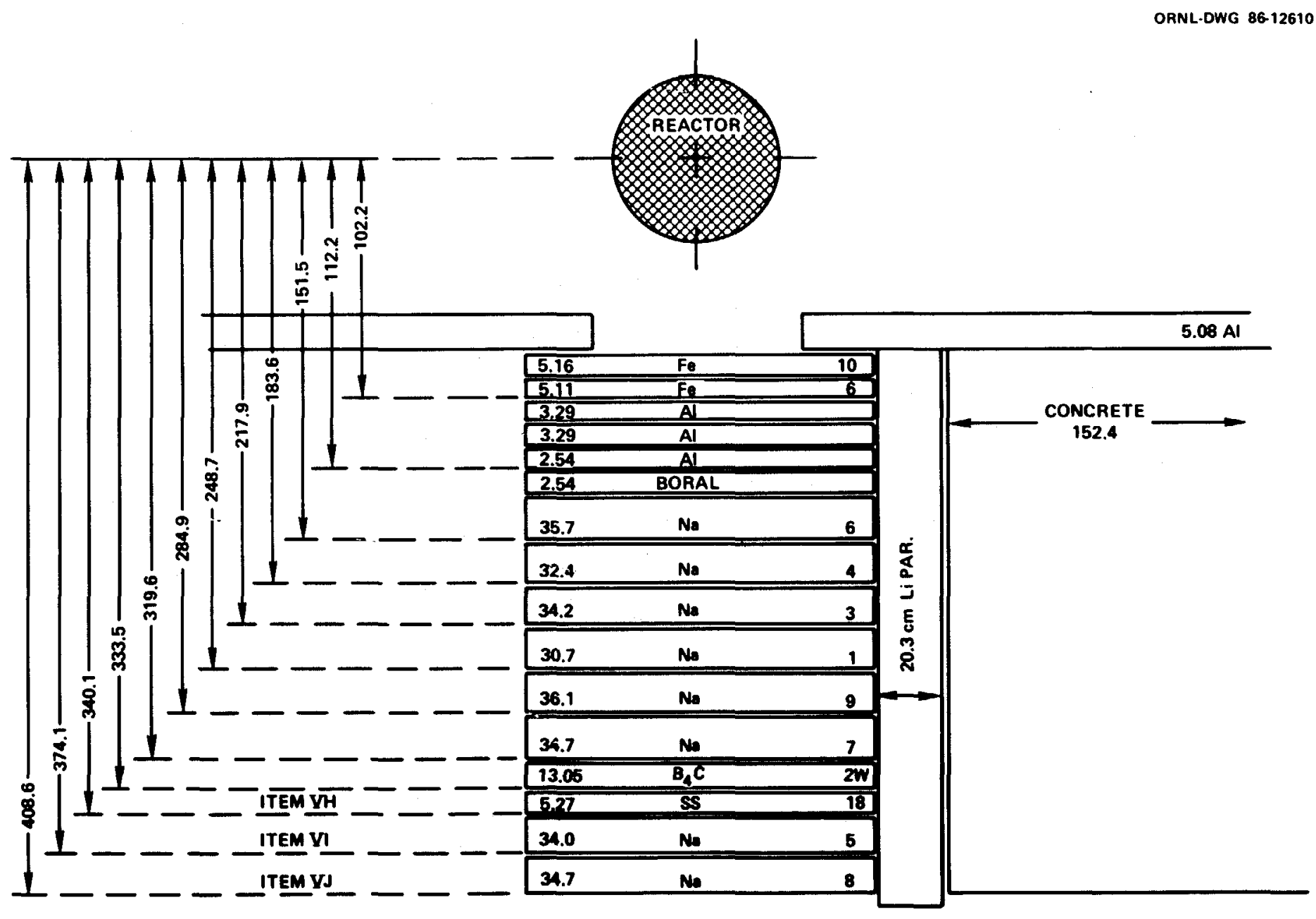

Figure 26. Schematic of SM2 plus shield configurations for Items $V H, I, J$. Note: Lithiated parafin covers lateral sides of conf igurations. 


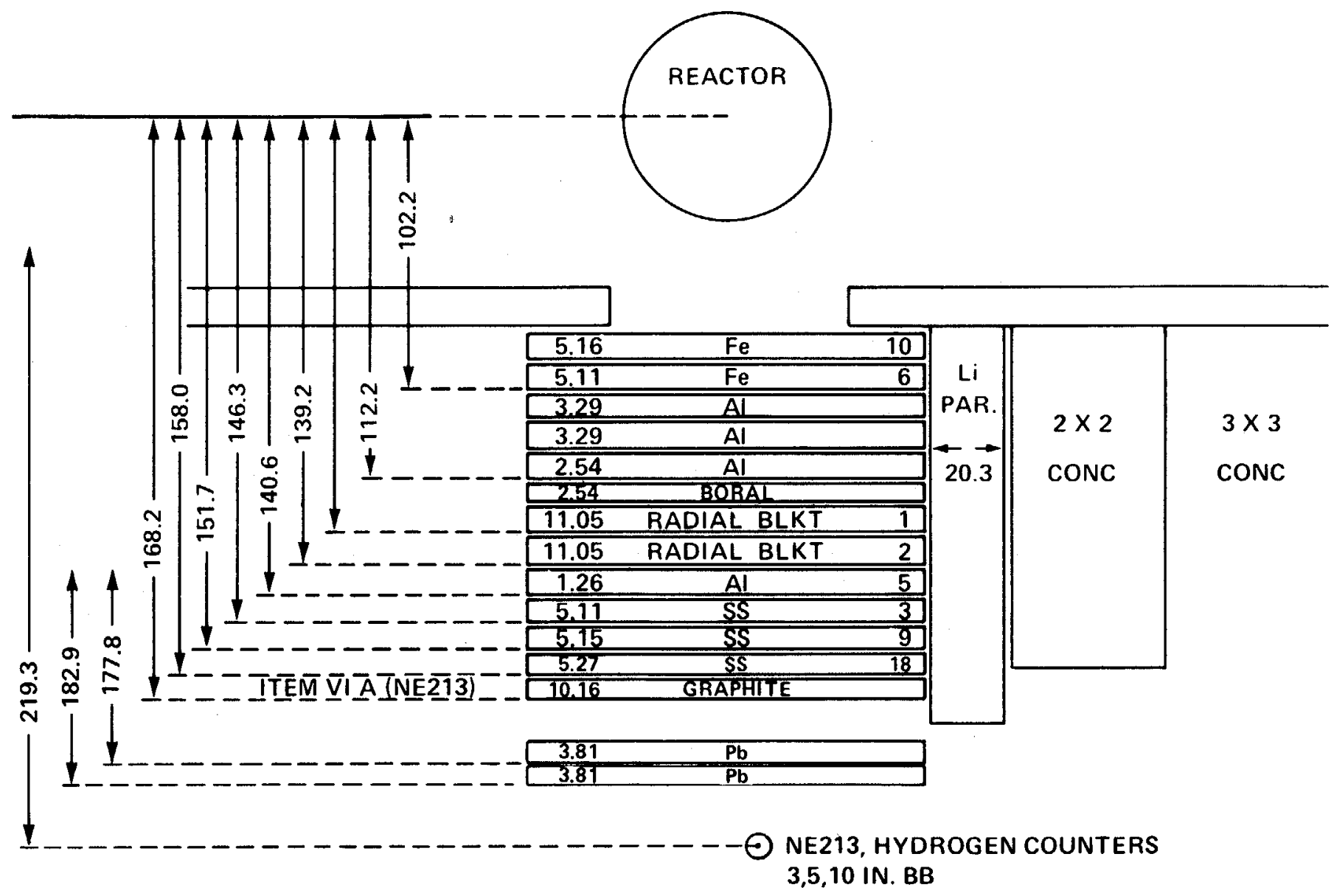

Figure 27. Schenatic of SM1 plus shield configuration for Item vIA. Note: Lithiated paraffin covers lateral sides of configuration. 


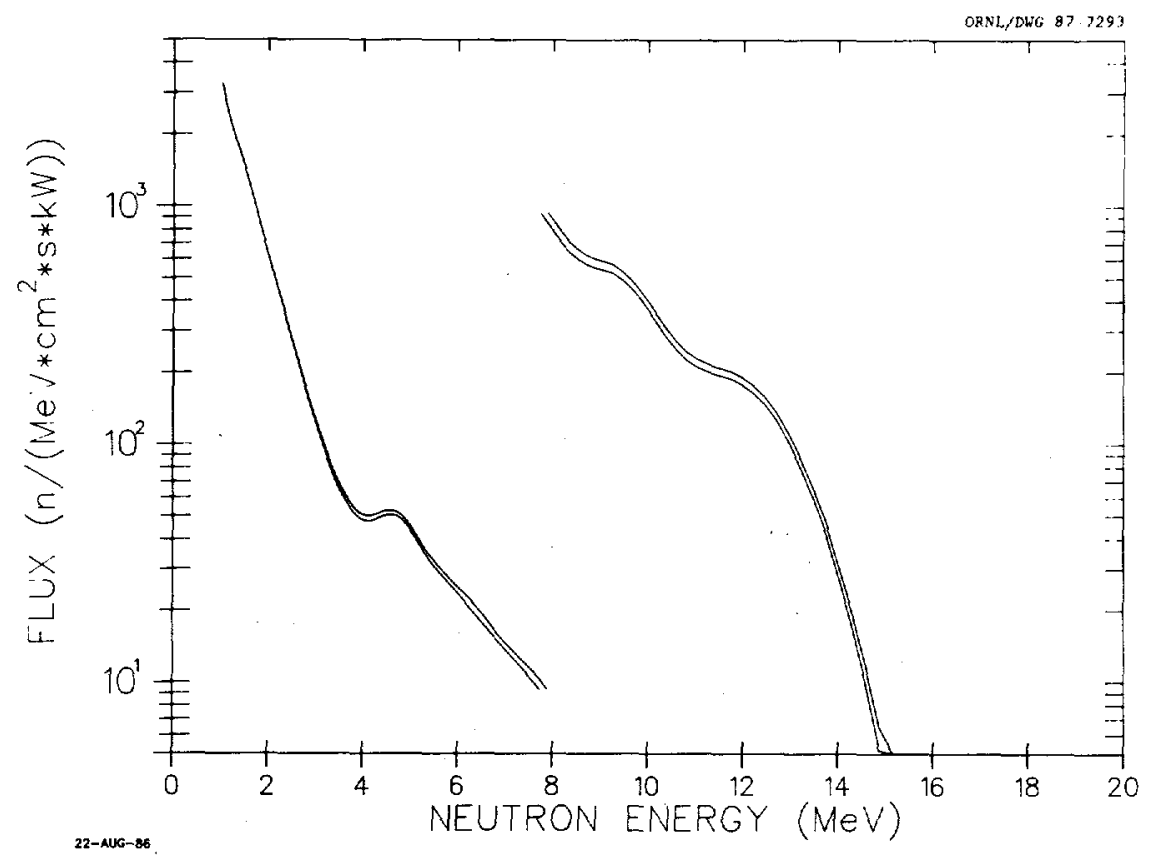

Figure 28. Fast neutron fluxes $(>0.8 \mathrm{MeV})$ on centerline at $51.1 \mathrm{~cm}$ beyond the graphite $(36.4 \mathrm{~cm}$ behind the lead) (Item VIA): Run 7858A.

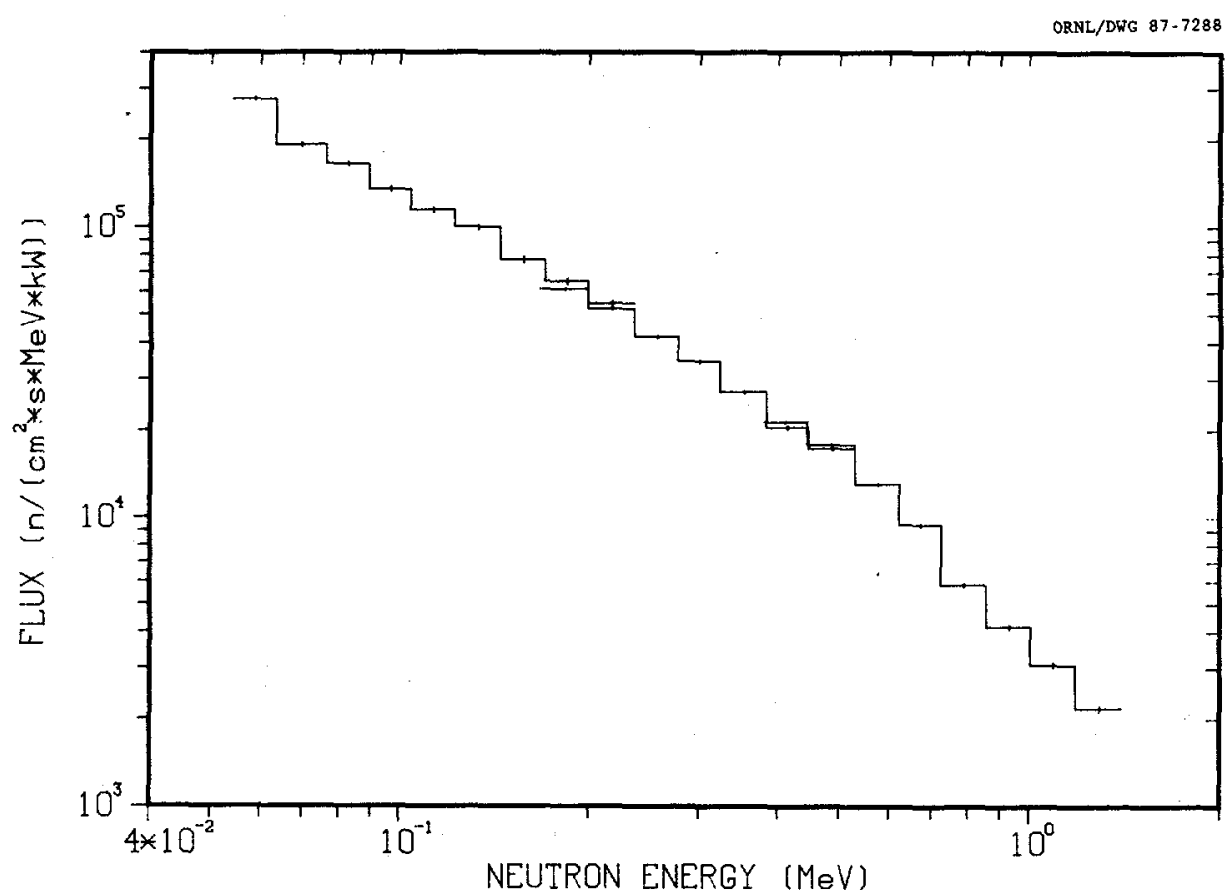

Figure 29. Neutron fluxes (50 keV to $1.4 \mathrm{MeV})$ on centerline at $51.1 \mathrm{~cm}$ beyond the graphite $(36.4 \mathrm{~cm}$ behind the lead) (Iten VIA): Runs 1542A, 1541B, 1541A. 


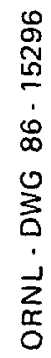

$\begin{array}{ll}m & 0 \\ \times & 0 \\ m & 0\end{array}$
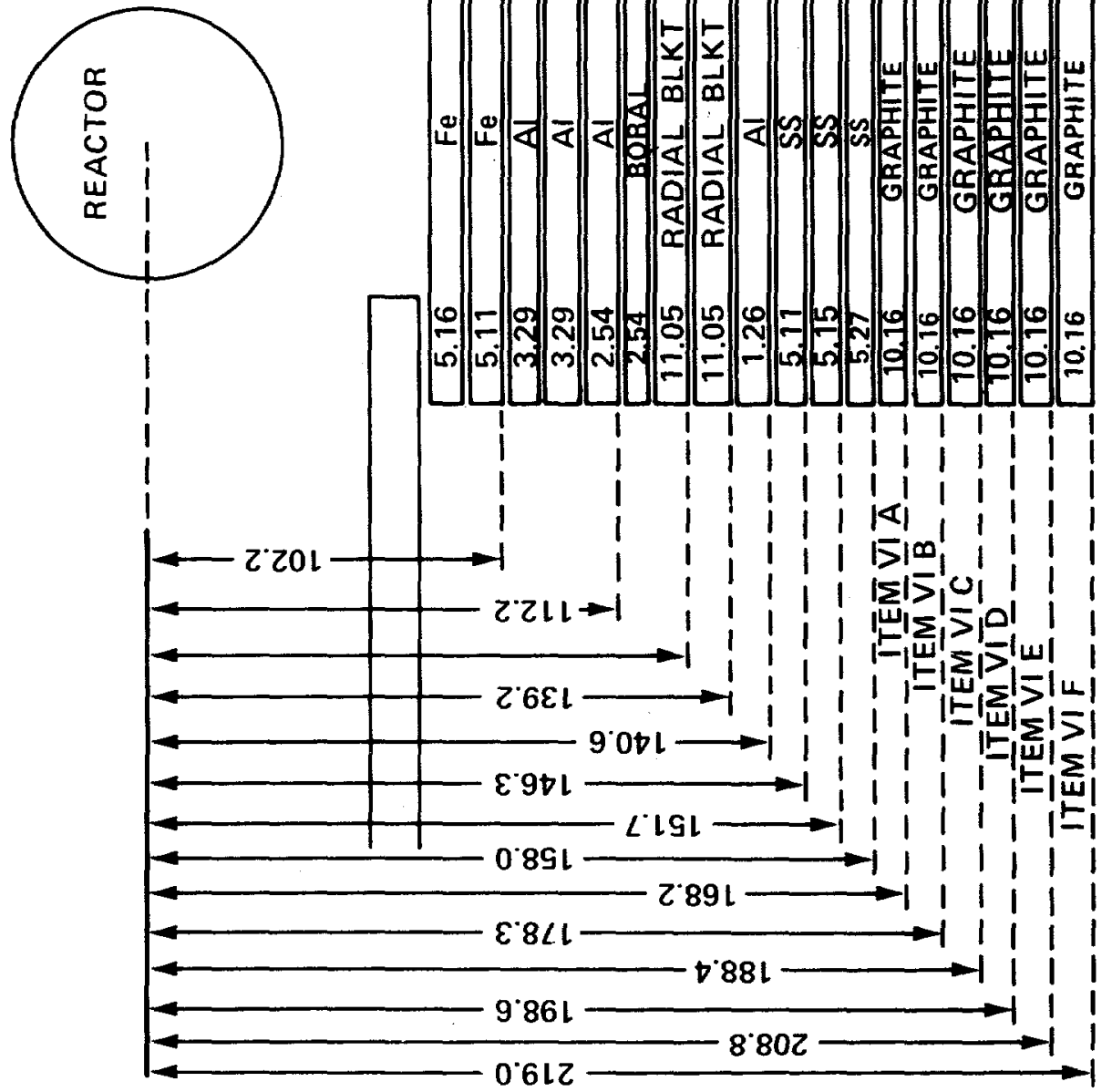

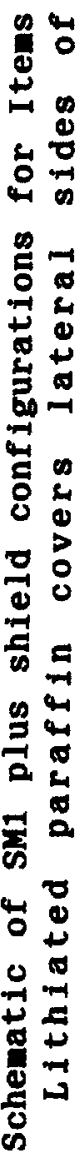

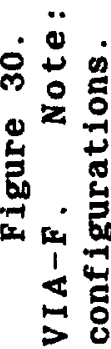




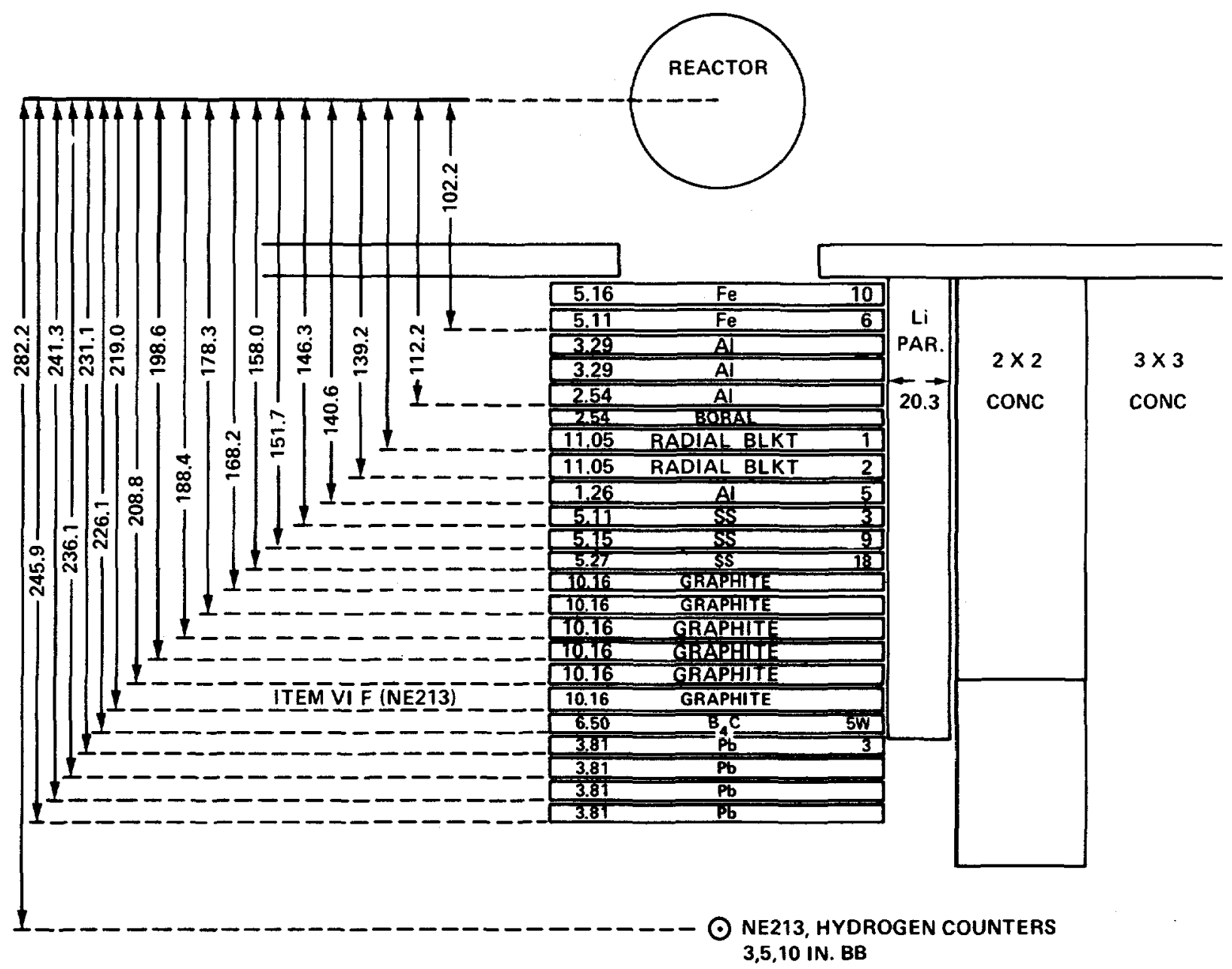

Figure 31. Schenatic of SM1 plus shield configuration for Item VIF. Note: Lithiated paraffin covers lateral sides of configuration. 


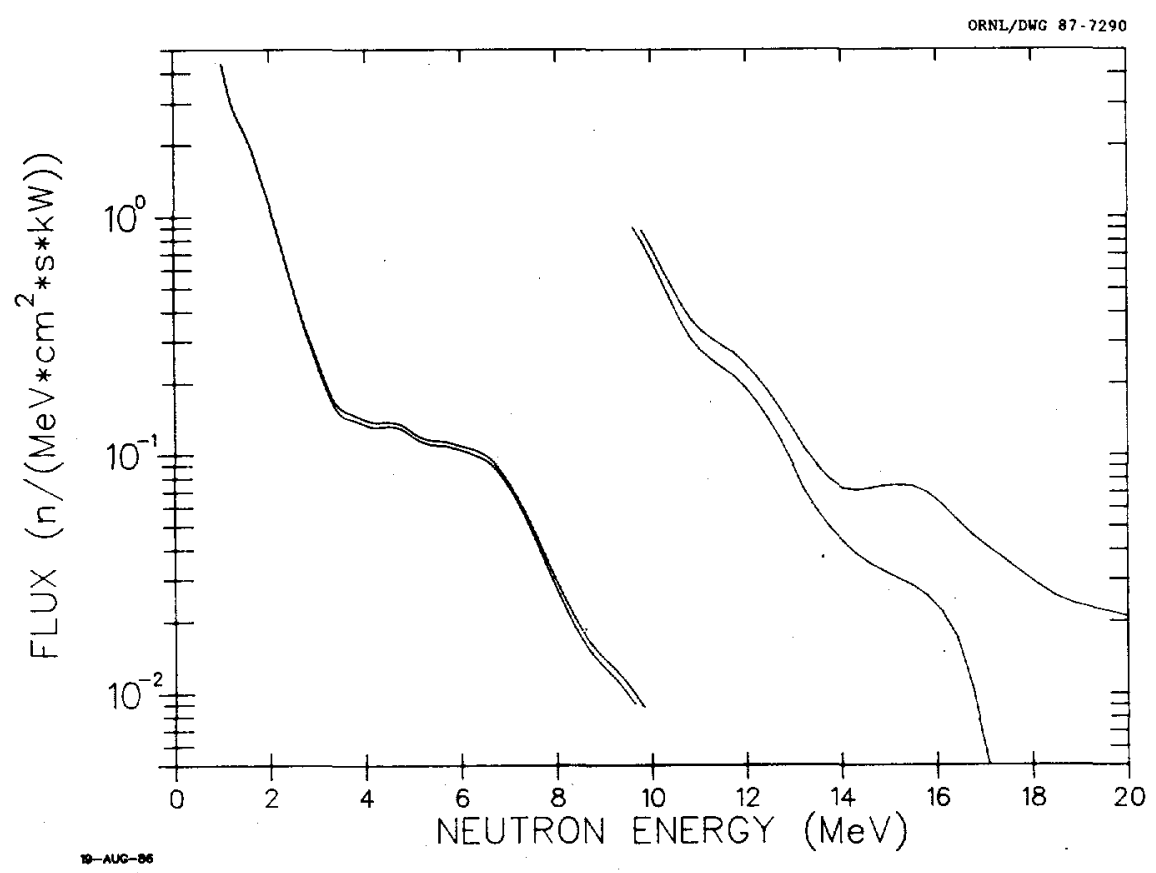

Figure 32. Spectrum of high-energy neutrons (>0.8 MeV) on centerline $63.2 \mathrm{~cm}$ beyond the graphite in the configuration (Iten VIF): Run 7859A.

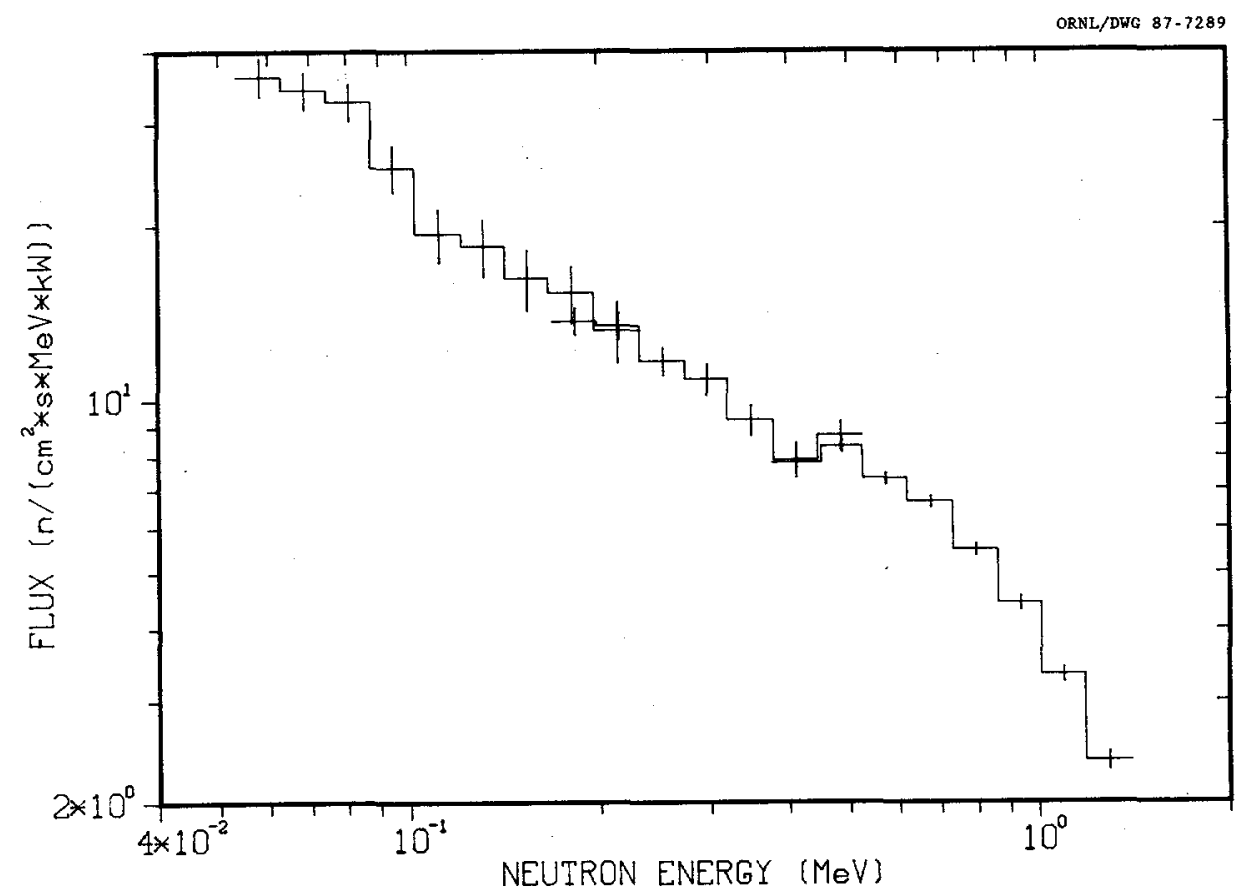

Figure 33. Neutron spectrun (50 keV to $1.4 \mathrm{MeV}$ ) on centerline 63.2 cm beyond the graphite in the configuration (Item VIF): Runs 1544A, 1544B, 1545A. 


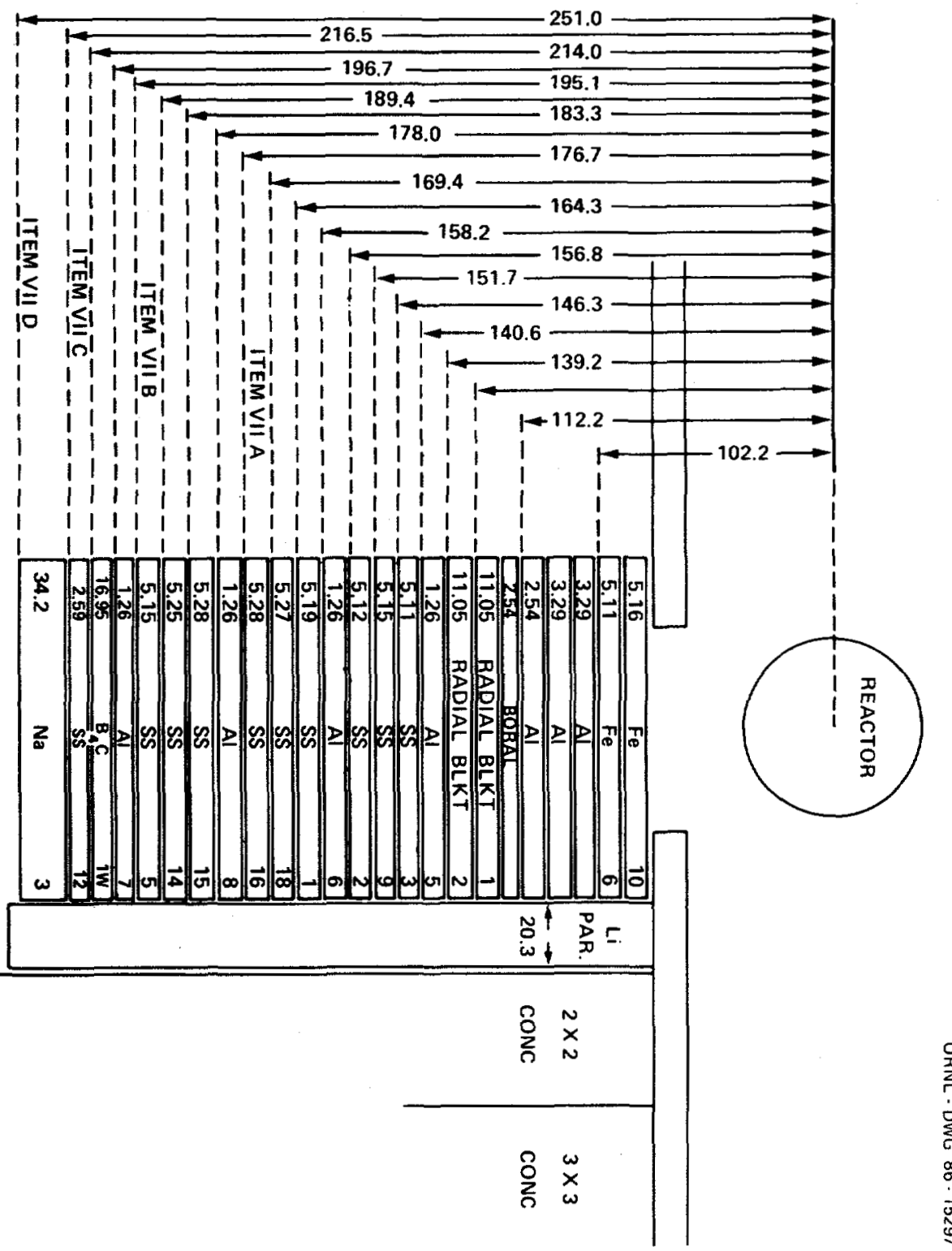

0
0
2
$\vdots$
0
$\vdots$
0
0
0
$\vdots$
0
0
0 


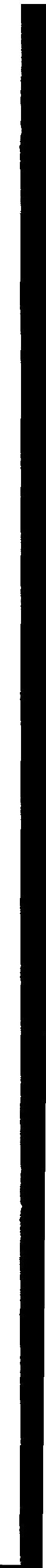




\section{INTERNAL DISTRIBUTION}

$\begin{aligned} \text { 1. } & \text { D. G. Cacuci } \\ 2 . & \text { R. L. Childs } \\ 3 . & \text { J. D. Drischler } \\ 4 . & \text { M. B. Emmett } \\ 5-8 . & \text { W. W. Engle. Jr. } \\ 9 . & \text { C. Y. Fu } \\ 10 & \text { G. F. Flanagan } \\ 11 . & \text { L. B. Holland } \\ 12 . & \text { J. } \text {.. Hull } \\ 13-17 . & \text { D. T. Ingersoll } \\ 18 . & \text { J. E. Jones, Jr. } \\ 19 . & \text { F. C. Maienschein } \\ 20 . & \text { J. J. Manning } \\ 21-25 . & \text { F. J. Muckenthaler } \\ 26 . & \text { F. R. Mynatt } \\ 27 . & \text { J. V. Pace, III } \\ 28 . & \text { R. W. Peelle } \\ 29 . & \text { W. A. Rhoades } \\ 30 . & \text { B. D. Rooney } \\ 31 . & \text { R. W. Roussin } \\ 32 . & \text { R. T. Santoro }\end{aligned}$

\author{
33. C. 0. Slater \\ 34. R. C. Ward \\ 35. C. R. Weisbin \\ 36. L. R. Williams \\ 37. A. Zucker \\ 38, P. W. Dickson, Jr. \\ (Consultant) \\ 39. G. H, Golub \\ (Consultant) \\ 40. R. M. Haralick \\ (Consultant) \\ 41. D. Steiner \\ (Consultant) \\ 42-43. Central Research Library \\ 44. Y-12 Technical Library \\ Document Reference section \\ 45-46. Laboratory Records \\ Department \\ 47. Laboratory Records ORNL, RC \\ 48. ORNL Patent office \\ 49. Engineering Physics \\ Information Center \\ 50-54. EPMD Reports office
}

\section{EXTERNAL DISTRIBUTION}

55. Office of the Assistant Manager for Energy Research and Development, Oak Ridge Operations, Department of Energy, P.O. Box E, Oak Ridge, TN 37830.

56. J. E. Brunings, Program Manager, SAFR, Rocketdyne Division, Rockwell International Corp., 6633 Canoga Avenue, Canoga Park. CA 91304 .

57. W. L. Bunch, Westinghouse-Hanford, 400 Area Trailer 1 , Richland, WA 99352 .

58 R. K. Disney, Westinghouse Electric Company, P.0. Box 158, Madison, PA 15663.

59. A. R. Gilchrist, Manager, Prism Technology Program, Nuclear Systems Technology Operation, General Electric Co., P.O. Box 3508, Sunnyvale, CA 94088. 
60 W. H. Harless, General Electric Company, P.0. Box 3508 , Sunnyvale, CA 94088 .

61. P. B. Hemmig, Advanced Technology Development, office of Technology Support Programs, U.S. Department of Energy, washington, DC 20545 .

62. R. J. Neuhold, Director, Advanced Technology Development, U.S. Department of Energy, Washington, DC 20545.

63. E. R. Specht, Rockwell International, 6633 Canoga Avenue, Canoga Park, CA 91304.

64-150. Given distribution as shown in TID-4500, LMFBR-Physics Applied UC-79-Td. 